\title{
From Location Description to Map \\ Understanding VGI from the Past
}

Gaurav Singh 


\title{
From Location Description to Map \\ Understanding VGI from the Past
}

\author{
Gaurav Singh
}




\section{PhD dissertation committee}

Chair

Prof. dr. ir. A. Veldkamp University of Twente Promoter

Prof. dr. M. J. Kraak University of Twente

Assistant promoter

Dr. ir. R. A. de By University of Twente

Members

Prof. dr. A. Voisard Freie Universität Berlin

Prof. dr. ir. M. F. A. M. van Maarseveen

University of Twente

Prof. dr. ir. M. G. Vosselman University of Twente

Dr. ir. M. van Keulen

University of Twente

ITC dissertation number 249

ITC, P.O. Box 217, 7500 AE Enschede, The Netherlands

ISBN: $\quad$ 978-90-365-3683-7

DOI: $\quad$ http://dx.doi.org/10.3990//1.9789036536837

Printed by: ITC printing department, Enschede, The Netherlands

(c) Gaurav Singh, Enschede, The Netherlands

(c) Cover design by Wim Feringa

All rights reserved. No part of this publication may be reproduced without the prior written permission of the author.

TC FACULTy OF GEO-INFORMATION SCIENCE AND EARTH OBSERVATION 


\title{
FROM LOCATION DESCRIPTION TO MAP
}

\section{UNDERSTANDING VGI FROM THE PAST}

\author{
DIS SERTATION
}

\author{
to obtain \\ the degree of doctor at the University of Twente, \\ on the authority of the rector magnificus, \\ prof. dr. H. Brinksma, \\ on account of the decision of the graduation committee, \\ to be publicly defended \\ on Thursday, June 5, 2014 at 14.45
}

by

\section{Gaurav Singh}

born on November 30, 1979

in Delhi, India 
This dissertation is approved by:

Prof. dr. M. J. Kraak (promoter)

Dr. ir. R. A. de By (assistant promoter) 


\section{Summary}

Location plays a fundamental role in human cognition and communication, certainly in this era of social media in which people have freedom to communicate anytime and from anywhere with current communication technology. This opportunity to communicate with text messages or through online social media such as twitter, blogs or facebook allows, for instance, sharing travel experiences.

Geographic Information Systems are well equipped to handle locational information expressed in longitude and latitude, however, they cannot convert the geographic information present in the text to useful map information. Such textual information is known to have existed for over 250 years with over a billion biological specimens collected, all providing information about the collection locality of the specimen, but not always providing coordinates. This is one of the barriers in using these descriptions for spatial analysis. With some effort of interpretation, one might be able to understand and geocode these locations. Geocoding from textual descriptions is important because it allows to address textual ambiguity and, once the geocoding is done, no other geographic identifier is required.

In a broader perspective the research project reported here aims to understand how humans communicate about location information using semi-structured text and how technology can aid in understanding and spatially representing it. For this purpose, real-world data from the published Ornithological Gazetteer of Brazil was used. In this gazetteer, localities are described using a number of statements that can be interpreted as spatial hints as to position. We identify those hints and their components, which need to be extracted and stored in a structured format. To do so, techniques of natural language processing and information extraction are used to understand the syntactic structure of the descriptions, based on which extraction patterns are developed per hint type. Upon extraction, these hints are translated to spatial representations.

Some hints allow us to represent crisp boundaries as vector representation, whereas others are represented using a probability raster approach. Using these two representation types, hints were converted into their relevant spatial representations and for an entry description, these were combined to derive the common area where the locality at 
hand is expected to fall. By carrying out this methodology for those entries with available geocodes, we are able to evaluate the accuracy of our results for this gazetteer. The approach presented in the thesis is generic and can be applied to other similar text sources. 


\section{Samenvatting}

Locatie heeft een belangrijke rol in menselijke cognitie en communicatie, vooral ook in het huidige tijdsgewricht waarin sociale media de mens de vrijheid verleent op ieder moment en iedere locatie te communiceren. Deze verworvenheid van communiceren met korte tekstberichten of via sociale media zoals twitter, blogs of facebook, stelt de mens bijvoorbeeld in staat reiservaringen uit te wisselen.

Geografische Informatiesystemen zijn uitstekend in staat met ruimtelijk informatie om te gaan als die is uitgedrukt in ruimtelijke coördinaten; ze zijn echter niet capabel in het omvormen van ruimtelijke informatie wanneer die besloten is in gewone tekst. Zulke informatie bestaat al minstens 250 jaar in de vorm van meer dan een miljard beschrijvingen van 'monsters'(verzamelde planten en dieren), waarmee in het algemeen een beschrijving van vindplaats is geassocieerd, echter niet altijd met echte ruimtelijke coordinaten. Dit is een belangrijke barrière voor ruimtelijke analyse van die beschrijvingen. Met de nodige inzet van interpretatie zijn zulke beschrijvingen echter wel ruimtelijk te duiden, via een proces van 'geocoderen'. Dat is een belangrijk proces omdat tekstuele ambiguíteit ermee geadresseerd kan worden, en vervolgens impliciete geografische referentie onnodig is geworden.

Vanuit een breder gezichtspunt poogt het onderhavige onderzoeksproject beter te begrijpen hoe menselijke communicatie ten aanzien van locatie plaats vindt, en hoe technologie kan helpen die communicatie te begrijpen en te representeren. Met dat doel zijn echte gegevens uit de gepubliceerde Ornithological Gazetteer of Brazil gebruikt. In deze gazet worden locaties beschreven middels een aantal deelzinnen, die interpretabel zijn als ruimtelijke hint. We identificeren deze hints en hun eventuele onderdelen, die worden geëxtraheerd en gestructureerd opgeslagen. Voor dat doel worden technieken van natuurlijke taalverwerking en informatie-extractie aangewend teneinde de syntactische structuur van de beschrijving te doorgronden met behulp van herkenningspatronen die per type hint werden ontwikkeld. Na extractie worden de gevonden hints in een ruimtelijke representatie opgeslagen.

Sommige hints kunnen het beste middels precies begrensde vectorgeometrie worden gerepresenteerd, terwijl andere hints beter representeerbaar lijken middels kansrasters. Gebruik makend van deze twee representatietechnieken werden ruimtelijke hints gevangen in een ruim- 
telijke representatie, en vervolgens geanalyseerd om een gebied van gemeenschappelijke dekking tussen hints af te leiden. Door deze methodiek toe te passen op beschrijvingen waarvan de locatie op andere wijze al bekend was, waren wij in staat de voorgestelde methode te evalueren op ruimtelijke precisie voor de gegevens in de gebruikte gazet. De beschreven methodiek is generiek en kan gebruikt worden voor andere bronnen van vergelijkbare aard. 


\section{Acknowledgments}

Like this thesis, our lives also have chapters and I am very glad that this important chapter in my life is finally coming to a close. The past five years have been most exciting, stressful and have made me a better person, I think. I thank God for giving me enough strength and courage throughout this PhD. During these years I got lots of love and support from my friends here and from back home.

Firstly, I am very grateful to my promoter Prof. Dr. Menno-Jan Kraak for promoting this thesis, and believing in me that this day will come. I am also very grateful to my co-promoter and supervisor Dr. ir. Rolf A. de By for his guidance and critical advise throughout this thesis. I learnt many things from him that has made me a better person and a researcher. Special thanks go to Arta Dilo and Ivana Ivánová who have shared their knowledge and expertise in critical domains of this research.

I want to thank all staff members of GIP department who have helped me at different point in time. I am grateful to have Bas Retsios as a friend who was always available to discuss small or big, technical or non-technical issues. He has helped me tremendously. Many thanks are also due to Barend Kobben, Ellen-Wien Augustijn, Martin Schouwenburg, Martin Molenar, Otto Huisman, Ulanbek Turdukulov and Rob Lemmens who have provided help or appreciated my research at various point in time. I am also grateful to Wim Feringa for designing the cover of this thesis on a very short notice in spite of the fact that he was busy with the teaching. I also appreciate and thank David Rossiter for sharing his knowledge on statistics and Dr. Claudia Robbi Sluter of Departamento de Geomática, Universidade Federal do Paraná for sharing her knowledge on Brazil.

Further, I would like to thank John Horn, Paul van Dijk and Loes Colenbrander for providing me full support right from joining the ITC till date. Thanks are also due to all staff members of ITC library especially Carla Gerritsen for arranging papers and books needed for my research on short notices. Many thanks are also due to ITC Students affairs, Marie Chantal Metz, Theresa van den Boogaard and Bettine Geerdink for their kind support on all matters related to IND or Gemeente. Special thanks to Marion Pierik for guiding me in the financial matters. Thanks are also due to all members of IT support department especially Aiko Mulder. Also to Roelof Schoppers for taking care of my lost pen-drive for almost 
a year and for greeting everyone with a happy face every morning and evening.

Thanks to all my PhD colleagues - Remi, Tanmoy, Paresh, Rishi, Rahul, Maitreyi, Divyani, Priya, Anandita, Bashar Alsadik, Mohammed Aljoufie, Alphonse, Frank Osei, Ahmed Ibrahim, Irma, Qiuju, Rehmat, Xiaojing and other ITC PhDs for their continuous support and encouragement. Thanks are also due to my very kind friends Rishin, Pramod, Amrish, Nirali, Vishakha, Chetna for their help and support. In this overwheming time of emotion, I may be forgiven for not being able to recollect those who have helped me now and then, but rest assured, my thanks to all those who have helped me complete this research by helping me in various tangible and intangible ways.

Yamini, my wife and my friend, has always been with me through thick and thin. While she has been supportive and understanding, she has also been my harshest critic and I wouldn't have it any other way. I will remember our brainstorming sessions over morning teas which she blackmailed me to prepare every morning. My daughter Rhea is the one who brings sunshine to my life every day and helps me see life from a different perspective! She is my life! I cannot thank enough my dear parents, for their endless love and prayers, who were always supportive of all my decisions including the one to leave my settled life in India behind and pursue a $\mathrm{PhD}$. My in-laws, my sister and brother in-law provided me with the emotional support and boosted my morale that kept me going through hard times.

In the end, I would like to thank European Commission Higher Education program for granting me the scholarship of to pursue PhD at ITC. It was their funding that got this started in the first place. I am also thankful to ITC Foundation for additional funding during the last stages of this research. 


\section{Contents}

Summary i

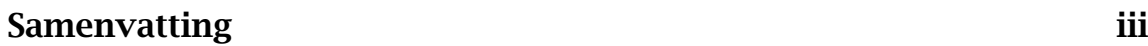

Acknowledgments $\quad$ v

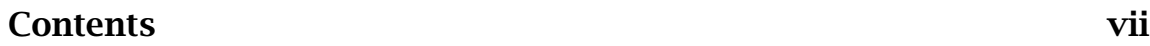

1 Introduction 1

1.1 Background and Motivation . . . . . . . . . . . . . 1

1.2 Research Objectives . . . . . . . . . . . . . . . . . . . . 4

1.3 Research approach . . . . . . . . . . . . . . . 5

1.4 Research relevance . . . . . . . . . . . . . . . 6

1.5 Thesis Outline . . . . . . . . . . . . . . . . . 6

2 Research corpus, its study and tools used 9

2.1 Introduction . . . . . . . . . . . . . . . . . 9

2.2 Structure of gazetteer entries . . . . . . . . . . . . . 11

2.3 Digitisation and storage of Gazetteer data . . . . . . . . 12

2.4 Spatial hints and their components . . . . . . . . . . 13

2.5 Dataset used . . . . . . . . . . . . . . . . . 17

2.6 Tools used $\ldots \ldots \ldots \ldots \ldots \ldots$

2.7 Summary $\ldots \ldots \ldots \ldots \ldots \ldots \ldots$

3 Extracting spatial hint information from locality descriptions 21

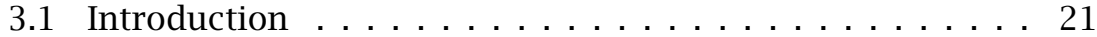

3.2 Related work . . . . . . . . . . . . . . . . . . . . 22

3.3 Approach and implementation . . . . . . . . . . . . . . 24

3.4 Description normalisation . . . . . . . . . . . . . 26

3.5 Natural Language Processing pipeline . . . . . . . . . . 28

3.6 Identifying subtree patterns in parse trees $\ldots \ldots \ldots$. . . 32

3.7 Information Extraction pipeline . . . . . . . . . 41

3.8 Evaluation and Results . . . . . . . . . . . . . . . . 45

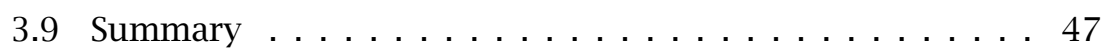

4 Spatial extent models for directional containment phrases 49 
4.1 Introduction . . . . . . . . . . . . . . . . 49

4.2 Related work . . . . . . . . . . . . . . . . . . 50

4.3 Background for constructing spatial extent models . . . . . 52

4.4 Construction of spatial extent models . . . . . . . . 55

4.5 Model performance and comparison . . . . . . . . . . 62

4.6 Data analyses . . . . . . . . . . . . . . . . . . . 69

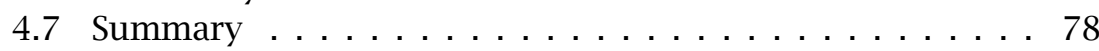

5 Spatial representation of hints using probability rasters 81

5.1 Introduction . . . . . . . . . . . . . . 81

5.2 Background on uncertainty . . . . . . . . . . . 82

5.3 Related work . . . . . . . . . . . . . . . . . . 86

5.4 Uncertainty in spatial hints . . . . . . . . . . . 88

5.5 General approach to probability raster creation . . . . . . 90

5.6 Raster creation for spatial hints . . . . . . . . . . . . 92

5.7 Optimisation of input parameters . . . . . . . . . . . 101

5.8 Raster overlay . . . . . . . . . . . . . . . . . . . 105

5.9 Overlaying rasters with vector model . . . . . . . . . . . 111

5.10 Summary . . . . . . . . . . . . . . . . . 112

6 Synthesis 115

6.1 Conclusions . . . . . . . . . . . . . . . . . . 115

6.2 Limitations . . . . . . . . . . . . . . . . . 118

6.3 Recommendations for future work . . . . . . . . . 119

$\begin{array}{ll}\text { Bibliography } & 121\end{array}$

$\begin{array}{ll}\text { A Appendix } & 131\end{array}$

B Appendix 135

$\begin{array}{ll}\text { C Appendix } & 137\end{array}$ 


\section{List of Figures}

1.1 Example entry from the gazetteer $\ldots \ldots \ldots \ldots \ldots \ldots$

1.2 Gazetteer entry with uncertain geocode $\ldots \ldots \ldots \ldots$

2.1 Gazetteer series published by Paynter and Traylor . . . . . . 10

2.2 Example entry from the gazetteer . . . . . . . . . . . 10

2.3 Example of a 'see' entry in the gazetteer . . . . . . . . . . 12

2.4 Gazetteer entries as in printed book and as digitised . . . . . 13

2.5 Example entry describing distance along/from a line . . . . . 14

2.6 Example entry describing distance/direction from a point . . . 15

2.7 Example entry for adjacency relation . . . . . . . . . . 16

2.8 Example describing direction containment relation . . . . . . 16

2.9 Example describing relative relations . . . . . . . . 17

3.1 Example entry from the gazetteer . . . . . . . . . . . . 21

3.2 The complete extraction pipeline implementation . . . . . . 25

3.3 Original and expected recognition of compound place name . 27

3.4 Place name recognition through a pattern $\ldots \ldots \ldots \ldots . . \ldots 27$

3.5 Output from the Stanford parser . . . . . . . . . . . 31

3.6 Example of four subtrees identified in a parse tree . . . . . 32

3.7 Parse tree showing matched nodes using patterns . . . . . . 36

3.8 Three nodes matched in a parse tree using different patterns . 37

3.9 Matched nodes in a parse tree . . . . . . . . . 37

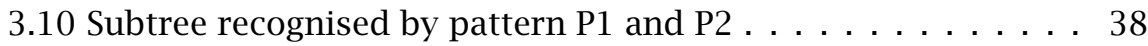

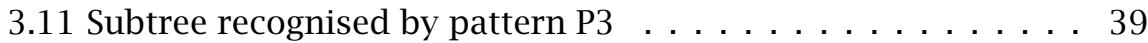

3.12 Subtree recognised by pattern P $4 \ldots \ldots \ldots \ldots$

3.13 Subtree recognised by pattern P5 . . . . . . . . . 40

3.14 Excerpts from a tree with relabeled nodes . . . . . . . . . . 43

4.1 Example entries from the gazetteer . . . . . . . . . . . 49

4.2 Hierarchy of directional containment . . . . . . . . . . . 53

4.3 Different types of central sectors $\ldots \ldots \ldots \ldots \ldots$

4.4 Different types of outer sectors . . . . . . . . . . . 58

4.5 Complete models for full directions . . . . . . . . . . . . 58

4.6 Different types of ' $\star c$ ' models $\ldots \ldots \ldots \ldots \ldots$

4.7 Different types of ' $c \star \star$ ' models . . . . . . . . . . . . . . 61 61

4.8 Different types of extreme direction models $\ldots \ldots \ldots 6$ 
4.9 Comparison of two full direction models . . . . . . . . . . 64

4.10 Performance of ' $\star c$ ' models at different centers . . . . . . . 66

4.11 Performance of ' $c \star$ ' models at different centers . . . . . . . 67

4.12 Performance of extreme direction models at different centers 69

4.13 Azimuth spread of full direction entries . . . . . . . . 70

4.14 Frequeny graph of full direction entries . . . . . . . . 71

4.15 Optimisation of size of central sector and outer sectors of full direction models . . . . . . . . . . . . . . . . . . 72

4.16 Frequency graph of half-direction entries $\ldots \ldots \ldots \ldots .74$

4.17 Azimuth spread of ' $\star c$ ' and ' $c \star$ ' entries $\ldots \ldots \ldots \ldots \ldots 75$

4.18 Spread of all half-direction entries . . . . . . . . . . 76

4.19 Azimuth spread of all half-direction entries . . . . . . . . 77

4.20 Azimuth spread of all half-direction entries for coastal and non-coastal states . . . . . . . . . . . . . 77

4.21 Frequency graph of extreme direction entries . . . . . . . 78

4.22 Azimuth spread of all extreme direction entries . . . . . . . 79

5.1 A conceptual model of uncertainty in spatial data . . . . . . 83

5.2 The effect of 'measurement' and 'conceptualisation' on uncertainty . . . . . . . . . . . . . . . 83

5.3 Example Normal distribution at different $\sigma$ values $\ldots . .884$

5.4 Example von Mises distribution at different $\kappa, \theta$ values . . . . 85

5.5 Illustration of five points of a raster cell . . . . . . . . . 91

5.6 Raster extent for 'distance direction from a reference point' hint type . . . . . . . . . . . . . . . . 93

5.7 Example raster for 'distance direction from a reference point' hint type . . . . . . . . . . . . . . . . . . . . 94

5.8 Example of 'distance along/from some linear feature' . . . . . 95

5.9 Raster extent for 'distance along/from some linear feature' hint type . . . . . . . . . . . . . . . . . . 96

5.10 Raster for 'distance along/from some linear feature' hint type 97

5.11 Example of 'between-two-points' hint type . . . . . . . . . . 98

5.12 Raster extent for 'between-two-points' hint type . . . . . . . . . 99

5.13 Raster for 'between-two-points' hint type . . . . . . . . . 100

5.14 Graph of weighted probabilities vs. $\sigma_{\text {dist }}, \sigma_{\text {dir }}$ combinations .102

5.15 Graph of weighted probabilities vs. $\sigma_{\text {dist_along }}, \sigma_{\text {dist_from }}$ com-

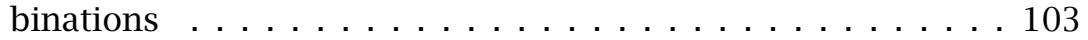

5.16 3D surface plot with peak of weighted probabilities . . . . . 105

5.17 Intersection of two events A and B . . . . . . . . . 106

5.18 An example illustration of raster overlay process . . . . . 108

5.19 Percent deviation in probability values over 39 of 46 resulting rasters . . . . . . . . . . . . . . . . . . . 109

5.20 Resultant rasters showing point $t c v(e)$ and $m c v(e)$ at different deviations . . . . . . . . . . . . . . . . . . . 109

5.21 Percent deviation of $t c v(e)$ from $m c v(e)$ is $51 \% \ldots \ldots \ldots . . .110$

5.22 Distances between $t c v(e)$ and $m c v(e)$ over 46 resulting rasters 110

5.23 An overlay of a resulting raster with vector model . . . . . . 112

5.24 An overlay of a single raster with vector model . . . . . . . 113 


\section{List of Tables}

3.1 Problematic elements present in gazetteer entries . . . . . . 26

3.2 Penn Treebank Part-Of-Speech tags . . . . . . . . . . . . . 28

3.3 Subtree structure of qualification hints in parse trees . . . . . 34

3.4 Some base expressions for tregex node relations . . . . . . 35

3.5 Direction offset hint table . . . . . . . . . . . . . . . 45

3.6 Distance direction from a reference point hint table . . . . . . 45

3.7 Pattern evaluation results . . . . . . . . . . . . . 46

4.1 Placement options for centre/central sector combinations . . . 56

4.2 Placement options for centre/outer sector combinations . . . . 58

4.3 Results of independent sample t-test on half-direction entries based on normalized distance to centroid . . . . . . . . 74

4.4 Results of independent sample t-test on half-direction entries based on azimuth differences . . . . . . . . . . . 76

A.1 Clause level Penn Treebank Part-Of-Speech tags . . . . . . . 131

A.2 Phrase level Penn Treebank Part-Of-Speech tags . . . . . . . . 132

A.3 Word level Penn Treebank Part-Of-Speech tags . . . . . . . . 133

A.4 Punctuation tags under Penn Treebank tagset . . . . . . . . 134

B.1 Tregex node-node relations and their symbols $\ldots \ldots \ldots 135$

C.1 Modularised parts of SHLP $\ldots \ldots \ldots \ldots \ldots \ldots$ 



\section{Introduction}

\subsection{Background and Motivation}

Geocoding is the process of assigning geographic coordinates to different forms of data, such as postal code, address, place name, place description and so on (Fu and Sun, 2011). Geocoding is a fundamental component for any kind of spatial analysis and is required in various application domains, such as emergency response, crime analysis, health, location-based services, SDIs, and business intelligence (Goldberg et al., 2007). Hence, it is an important process to geocode and map real-world geographic features. By geocode we mean geographic coordinates of the locality. The information on these features is available in various forms and through various sources.

In the last decades, advancement in communication technology (esp. internet and telecom) has paved the way for people to communicate anytime, from anywhere and about anything. Information (esp. location information) has become pervasive, be it through sharing photos on flicker, walking/travel tracks using GPS, or receiving directions through navigation devices. Another method of sharing information is through text messaging like Short Messaging Service (SMS) or through online social media such as Twitter, blogs or Facebook which provide ample opportunity to users for sharing information in real time and on-themove. For instance, users often post messages like 'gliding in northern France', 'epicenter of earthquake $150 \mathrm{~km}$ NE of Delhi' or 'halfway between Enschede and Deventer'. These text phrases do not contain any geocode nor come with any GPS tags, yet provide important locational information. Geocoding from textual descriptions is non-trivial and important because it bridges text and spatial intelligence, and allows to put described places 'on the map'. The information shared through textual descriptions may broadly come in two kinds:

- Those applying a textually formalized format of some kind (semistructured text ${ }^{1}$ ), and

- Those using really casual text with slang words, abbreviations and so on.

\footnotetext{
${ }^{1}$ By semi-structured text we mean text data that follows some standard structure but does not conform to the formal structure of the data models used in databases.
} 
It is somehow difficult to handle casual text because of syntactic variability, the use of informal jargon and abbreviations that may vary with person and place. However, in comparison with casual text, working with standardised semi-structured text is easier and allows for improved understanding of how humans communicate locations using natural language.

Even in the past (i.e., pre-GPS days), when the media for collecting and sharing information were limited, locations and their names, were recorded as textual descriptions. As per Beaman et al. (2004), it is believed that natural history expeditions over 250 years have collected over a billion biological specimens, all providing information about the locality where the specimen was collected, but not always providing coordinates, which is one of the barriers in using these descriptions for spatial analysis.

An interesting case with relevance to the present time is formed by localities visited by natural historians, which later were aggregated in a geographical dictionary, called a gazetteer. A gazetteer is a geographical dictionary that contains place names and provides, where possible, a geocode for each of those (Hill, 2006; Vestavik, 2004). Not every place name may be a known locality, and then it may be described with reference to relative positions of other geographical features such as (Stuckenschmidt et al., 2001):

- Human dwellings (i.e., cities, villages, towns, farms)

- Man-made objects (i.e., bridges, railroads, tracks, and trails, and so on)

- Natural objects (i.e., lakes, rivers, waterfalls, forests and mountains)

- Administrative units (i.e., countries, provinces and regions)

- Spatial relationships (i.e., distance to objects, neighboring geographic feature, contained in and so on)

- Topographic features (i.e., elevation, direction and location reference)

- Demographic details (i.e., population)

- Events (i.e., floods, fires, or disease outbreaks)

- Bibliographic references (i.e., reference of a spatial object taken from a particular book, maps or any other source)

Any description will contain a number of statements/phrases that can tell about the whereabouts of the locality being described. For instance, in ornithology, a series of gazetteers was published by Paynter and Traylor in the 1980s-1990s (Paynter and Traylor, 1991a,b). An example entry from these is provided in Figure 1.1. In this description, the location of 'ABRILONGO' is indicated as unknown. There are, however, hints in the description like elevation, distance from another locality, and reference to published papers that may help in determining position of the locality with some level of accuracy. The problem is to determine a geocode or improve on an existing geocode, and determine a notion of accuracy as 
all hints come with a different accuracy. The question here is how we can quantify these, and use them to determine accuracy of the overall geocoding process.

ABRILONGO; Mato Grosso Not located

ca. I,500 ft [460 m], $12 \mathrm{mi}\lfloor 19 \mathrm{~km}\rfloor$ from [direction ?] Chapada

[ca. I555/5625], campos of southwestern Mato Grosso, Smith, et al.,

ca. I 882-I 886 , with known dates of Feb. I883, 8, 20 Feb. I885, I 8,

20, 23 Feb., 2 Mar. year ? or years ? (Allen, I891; I892; 1893).

Figure 1.1 An example entry from the gazetteer (Paynter and Traylor, 1991a), describing the locality Abrilongo

A number of difficulties can arise in the process of geocoding from textual descriptions. Some of them have been listed below:

- Locality description may have no geocode, uncertain or a wrong geocode. For example, in Figure 1.1 locality 'ABRILONGO' has no geocode and is marked as 'Not Located'. Similarly, in Figure 1.2 locality 'AREIÃO' has uncertain geocode 'ca. 1002/4100' since it mentions 'ca. ${ }^{2}$ ' before its geocode.

- Use of incorrect directional references in locality descriptions. For example, locality is in east part-of some state, but referred as in west part by the author in the description.

- Author of locality description may have erred in use of an object name inside the description. That name may identify a different object, or may not identify any object at all.

- Typing mistakes may obfuscate the true intention of the author.

- Author may blindly have followed previous authors and propagate historic error.

- Change in spatial relationship like distance to objects (lake shrunk, cities grow, rivers change course), neighboring geographic features or changes due to changed administrative boundaries.

- Ceased existence of previous natural objects (which might have diminished over the period of time) like lakes, rivers, waterfalls, forests, tracks and trails, and so on.

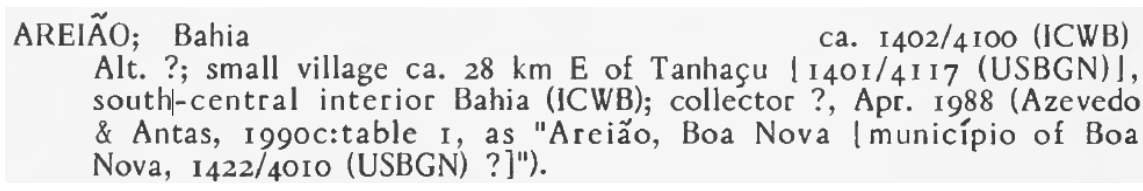

Figure 1.2 An example entry from a gazetteer (Paynter and Traylor, 1991a), describing the locality AREIÃO with its uncertain geocode

With some effort of interpretation, one might be able to geocode these locations. Geocoding from textual description is important because it

\footnotetext{
${ }^{2}$ ‘Ca.' is an abbreviation for Latin word 'circa' which means 'approximately' in English.
} 
removes textual ambiguity and once the geocoding is done no other geographic identifier is required.

In the following section we discuss the research objectives followed by research approach in Section 1.3. In Section 1.4, we discuss research relevance after which thesis outline is discussed, in Section 1.5.

\subsection{Research Objectives}

The research project presented in this thesis investigates geocoding from text data. The general objective of this project is 'improving our understanding of how humans communicate locations using text and geocoding such semi-structured textual descriptions that may have unknown, uncertain or even incorrect geocode'. As discussed earlier, a historical gazetteer contains such textual descriptions of localities. Hence for this project our research corpus will be a historical gazetteer, more specifically the ornithological gazetteer of Brazil written by Paynter and Traylor (1991a,b). This is done by keeping in mind the following sub-objectives:

- To understand the textual structure of gazetteer entries and identify hints and their components that can be exploited spatially,

- To identify and exploit syntactic structure of hints by developing patterns for extraction and storage of hints in a suitable data structure for efficient storage and retrieval,

- To identify types of uncertainty that occur in our spatial hints,

- To develop vector-based or raster-based spatial representations for spatial hints,

- To predict area of certainty by combining raster-based and vectorbased spatial representations for various entry descriptions to derive an area of certainty in which the locality is expected to fall.

The gazetteer descriptions describe the cognitive conceptualization of the locality as perceived by explorers. The textual structure of these descriptions are easier to understand by humans but are difficult to be exploited by machines, yet still allow, for various spatial analyses or computations to be carried out. We believe that before any computation can be realized, an extraction of information from these hints and storing them into structure entities is required. Since these descriptions are represented as natural language text, it is imperative to identify and understand types of uncertainties associated with them. For example, when describing Hengelo city, which is 'ca. $8 \mathrm{~km}$ NW of Enschede', there may be three uncertainties associated with it, i.e., distance uncertainty, direction uncertainty and others. It is important to include them when geocoding from localities descriptions (Guo et al., 2008; Liu et al., 2009; Wieczorek et al., 2004) or when carrying any spatial analysis without which the process of geocoding and analysis is of limited use (Fisher, 1999). Some hints may be represented by crisp boundaries for spatial representation, while others need probabilistic raster approach (Guo 
et al., 2008) for their representation. These rasters can then be combined for set of hints per gazetteer entry, to predict area of certainty where a locality can be found. This approach also allows us to find presence of conflicting hints, wrong hints and level of accuracy for entries in our gazetteer.

\subsection{Research approach}

The research corpus is a two volume printed gazetteer (Paynter and Traylor, 1991a,b), which is digitised using optical character recognition (OCR), after which all entries are corrected for errors induced through OCR processing. The entries are then stored in a simple data structure in a PostgreSQL database. The textual structure of the gazetteer entries is identified, which broadly consists of elements such as place name, state name, geocode of the place and description about the place. A locality description contains a number of statements that can be interpreted as spatial hints to its position. We then identify those hints and their components as present in it.

In order to do any spatial analysis or computations with spatial hints, we first need to extract those hints and store them in a structured format in a database. This falls in the domain of information extraction through natural language processing. In this, a natural language description is parsed through the text processing tool which works on the grammatical structure of the description and produces a tree structure in which each text in the description is assigned to its most probable grammatical tag. For our parsing needs, in this project, we will use the Stanford parser ${ }^{3}$ from Stanford university. From the output of the parser we will identify the most commonly occurring sub-tree structures in the descriptions. These structures were then used to develop patterns, which will then be used for extraction and storage of information in a database.

Since these hints represent the spatial perceptions of humans, about the locality, these hints come with some level of uncertainty associated with them. We want to translate these hints in to spatial representations, by which, we mean how they can be represented on a map. For example, for a phrase 'southern part of some region' we want to represent the boundary of 'southern part' of that region within which that locality can be located. Some hints allow us to represent crisp boundaries as a vector representation, whereas others do not. For the latter, we use probability raster approaches as suggested by Guo et al. (2008) and Liu et al. (2009). To convert as many hints in to their spatial representations, we want to derive best-as-possible raster representation along with the measure of associated uncertainty. Later, for an entry description we will combine specific hint rasters and define an area of certainty. Further evaluation of rasters will allow us to find inconsistent hint types and the accuracy

\footnotetext{
${ }^{3}$ The Stanford parser:http://nlp.stanford.edu/software/lex-parser.shtml
} 
with which this area of certainty is able to predict the location of target locality.

\subsection{Research relevance}

This research project aims to transform locality descriptions to geographic information. With successful completion of the project, our methodology can be extended to other gazetteers by the same authors and even to other contexts. One of the other contexts is the Global Biodiversity Information Facility ${ }^{4}$ (GBIF) database where this process of geocoding can be used to create geoinformation. Geocoding implicit text data from historical perspective also becomes possible with our methodology. In the present scenario, our results can be applied to Location-Based Services (LBS), Geographic Information Retrieval (GIR) and geocoding textual data available on various social networking sites such as Twitter, Facebook, blogs, and so on. Additionally, diverse subjects such as archeology and biological studies also stand to gain from the results of this project.

\subsection{Thesis Outline}

This section discusses the underlying structure of this thesis:

Chapter 1 (this chapter) provides general introduction to this research project, including background and motivation, research objectives, its scope and limitations, research approach and thesis outline.

Chapter 2 gives a general description of the data used for this research. In this chapter, the general structure of the gazetteer entries and various spatial hint types and their main components are discussed. Also discussed is the support datasets and tools used to carry out the project.

Chapter 3 discusses the need for information extraction from spatial hints. The approach and experimental implementation along with detailed steps used in extraction is discussed in this chapter. Later in the chapter we have also discussed the evaluation method adopted for our extraction process and finally the results of it.

Chapter 4 in this chapter we present computational mechanism to allow the interpretation of text phrases such as "in southeastern Bahia," "in central Goiás," "central northern Pará," and "extreme northern Bahia" as spatial features in GIS context. Construction of different plausible vector-based spatial representations for these phrases is discussed in detail. We call these representations as models. We

\footnotetext{
${ }^{4}$ GBIF is an organisation established in 2001 for open access to biodiversity data through Internet (http://www.gbif.org/). Data available on GBIF are for studies of species, ecological, molecular, and scientific names data.
} 
subsequently evaluate those models for the performance characteristics, against our gazetteer corpus, and draw conclusions on the best-as-possible spatial representation for each type of text phrases mentioned above.

Chapter 5 provides insight to spatial uncertainty associated with other types of hints. We also discuss the creation of probability rasters for different hint types. The rasters for various spatial hints in an entry are also overlaid to determine an area of certainty where the target locality can be found. We also overlay these rasters with vector models as created in Chapter 4 and evaluated the performance and consistency of both raster and vector-based spatial representations of hints.

Chapter 6 summarises the thesis, provides conclusions, limitations and recommendations for future work. 



\section{Research corpus, its study and tools used}

\subsection{Introduction}

A gazetteer is a geographical dictionary that contains place names and provides, where possible, a geographic coordinate for them (Hill, 2006). Gazetteers play an important role in organising and maintaining placerelated knowledge. In the context of current times, gazetteers, such as geonames $^{1}$, Alexandria Digital Library ${ }^{2}$, Getty Thesaurus of Geographic Names $^{3}$ and many others help in linking geographic text (e.g., news articles) with relevant map information. However, when indirect references to places are mentioned, most gazetteers are only of limited use in providing map information. Phrases like ' $10 \mathrm{~km}$ NE of Enschede' or 'eastern part of Ohio' are important to understand and geocode as they can provide useful information about a locality that may be of interest to a person or specific communities, such as natural historians, ornithologists or archaeologists.

The gazetteers are known to have existed as early as $6^{\text {th }}$ century in which, the place names and their descriptions were recorded (Hill, 2006). In ornithology, many gazetteers were published from 1970s through early 1990s by Raymond A. Paynter, Jr. and Melvin A. Traylor, Jr. for South American region. They published eleven gazetteers for countries like Bolivia, Ecuador, Colombia, Uruguay,Venezuela, Peru, Argentina, Guianas, Chile, Praguay and Brazil. Their gazetteer series (Figure 2.1) contains more than 3,000 pages that compiles localities mentioned in ornithology literature along with hundreds of unpublished localities (AOU, 2002).

We use the Ornithological Gazetteer of Brazil written by Paynter and Traylor (1991a,b), which is a good corpus to work with because its descriptions are semi-structured and it shows less variability in its language usage than casual text data. The book contain entries from expeditions dated back to 1800 . The authors compiled this gazetteer by hand by double-checking thousands of original scientific publications (AOU,

\footnotetext{
${ }^{1}$ http://www.geonames.org/

${ }^{2}$ http://www.alexandria.ucsb.edu/

${ }^{3}$ http://www.getty.edu/research/tools/vocabularies/tgn/
} 


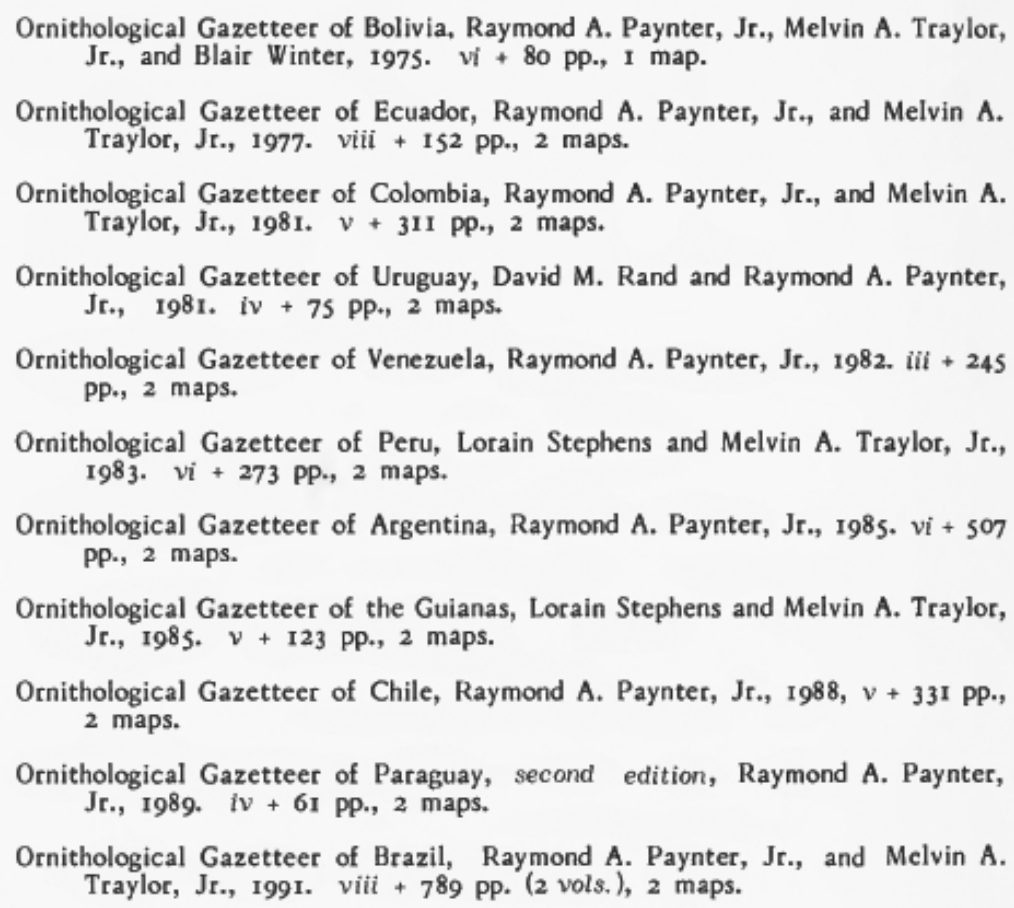

Figure 2.1 Gazetteer series published by Paynter and Traylor

2002). Figure 2.2 shows an example gazetteer entry with locality name in capital letters and its description.

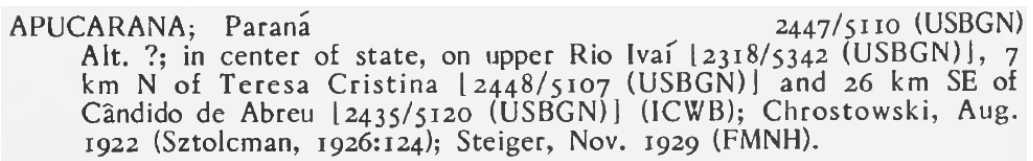

Figure 2.2 An example entry from the gazetteer (Paynter and Traylor, 1991 a)

The gazetteer contains over 8,000 entries, covering roughly 6,000 sites visited and surveyed by ornithologists. Amongst these, there are around 12\% localities that are identified as 'Not Located', $47 \%$ are 'see entries' that refer to descriptions of other entries in the gazetteer, and a further 8\% have an uncertain geocode (Paynter and Traylor, 1991a,b) as shown in Figure 1.2.

Our interest in this gazetteer comes with a reason. Only $18 \%$ of the Brazilian data held in the Global Biodiversity Information Facility (GBIF) on avian taxa is geocoded. Brazil contributes significantly to the GBIF since it is globally the country holding the largest biodiversity. The Paynter and Traylor gazetteer series is considered a seminal work in the domain of Ornithology (AOU, 2002) and is the single most important 
resource for Brazil. Hence, it becomes a natural choice to try geocode entries by this gazetteer, eventually adding to the geocoded data held in GBIF. All entries describe natural historical collection sites of biological expeditions and exhibit strong spatio-temporal relationships in the descriptions.

In this chapter, we study the structure of the gazetteer entries, by finding most commonly occurring phrases with which spatial computations and analysis can be carried later on in this research project. In Section 2.2, we identify the structure of the gazetteer entries. We then describe the need for digitisation and storage of gazetteer data in Section 2.3. Various spatial hints and their components are identified in Section 2.4, followed by datasets and tools used, in Section 2.5 and Section 2.6 respectively.

\subsection{Structure of gazetteer entries}

The gazetteer follows a semi-structured content structure, which is useful for our understanding of its textual components. With reference to the original text in Figure 2.2, a gazetteer entry in principle consists of English phrases or sub-phrases, however, with Brazilian Portuguese names. Typical components of an entry are:

Place name: Displayed in full capitals is the name of the place that the entry describes ('APUCARANA'). To serve alphabetization, names of bays, rivers, states and a few more are listed in a comma-separated format, with the primary name put first, as in 'CALOR, RIO DO'.

State name: Features the name of the state ('Paraná') in which the place is (assumed to be). In some cases, multiple states are listed.

Geocode: In most cases, this is a one arcminute accurate geocode with a source indicated, such as in ' $2447 / 5110$ [USBGN]'. This informs us of latitude/longitude, by default south and west, in degrees and minutes, here according to the United States Board on Geographic Names ('USBGN'). In our example, the geocode is $S 24^{\circ} 47^{\prime}, W 51^{\circ} 10^{\prime}$. When uncertain, the geocode may be tagged with the word 'ca.'. When unknown, the locality is indicated as 'not located'. In some rare cases, larger areas are geocoded with a pair of geocodes, implying the lower left and the upper right corner of a bounding box. All of the above text parts are placed on the first line of the entry in the printed gazetteer.

Spatial description: This is an English semi-sentence, often lacking conjugations of the verb 'to be' that typically describes the locality, and provides a small number of hints to 'put the described place on the map'. The hints themselves come from older literature, cited in the historical description.

Historical description: This is second semi-sentence, separated from the spatial description often by a semicolon, which contains references to expeditioners that have visited the place, the date(s) 
of their visit, as well as literature references that report on those historic visits. Our example in Figure 2.2 shows one such reference, namely 'Sztolcman' writing in '1926' about 'Chrostowski' visit in 'Aug. 1922' and an additional reference to a visit by 'Steiger' in 'Nov. 1929' from Field Museum of Natural History 'FMNH'.

Separators between these respective text parts are often obvious, being semicolons or commas, but occasionally are more problematic to identify, having been omitted or with the default semicolon replaced by a colon. The latter is especially the case between the spatial and historic components of the description. These two occasionally come in reversed order, which is a slight further complication. The gazetteer also has additional entries than those described above. One important class of these are the so-called 'see entries', which provide alternative names to already listed entries. The alternative names are referred as endonym ${ }^{4}$. These alternative names often stem from literature sources, old charts and maps, and may reflect different names at time of writing, or simple typographic errors. For example, Figure 2.3 shows two alternate names for river 'Feio, Rio', first name 'AGUAPEÍ, RIO' from International Chart of the world on the Millonth Scale, Brazil ('ICWB') and second name 'AGUAPEHY, RIO' from Map of Hispanic America ('MHA').

$$
\begin{aligned}
& \text { AGUAPEI, RIO (ICWB); see Feio, Rio. } \\
& \text { AGUAPEHY, RIO (MHA); see Fcio, Rio. }
\end{aligned}
$$

Figure 2.3 An example 'see' entry from the gazetteer (Paynter and Traylor, 1991a) showing alternative spellings for river 'Rio Feio'

\subsection{Digitisation and storage of Gazetteer data}

To use gazetteer for any meaningful geo-computation or spatial analysis it needs to be in digital form and stored in a structured way. For digitisation we use Optical Character Recognition (OCR) technique, which is a common mechanism for digitising the texts. Since our gazetteer is written in English with Brazilian-Portuguese place names containing accented characters, some OCR errors occured as it was not able to recognise such characters. Correction to the digitised data was done manually for all entries as it can not be done automatically. Figure 2.4(a) shows the original page from the gazetteer and Figure 2.4(b) its digitised version, which reflects the kind of errors observed. For example, the geocode 'ca. 0100/4900' appeared as 'ca.oioo/4900', and the locality name 'MAGÉ' appeared without accent after digitisation.

Once we corrected all the errors in the digitised data we created a simple data structure for storage of this data in PostgreSQL database.

\footnotetext{
${ }^{4}$ When the toponym is mentioned in local language.
} 


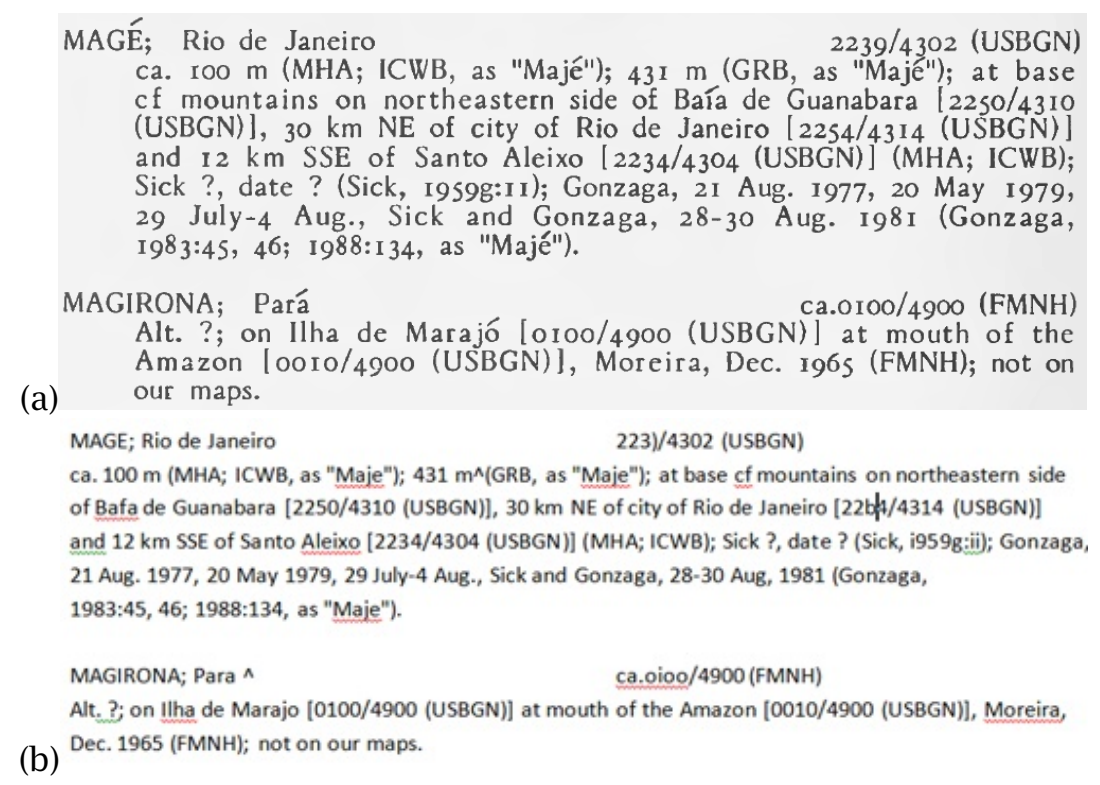

Figure 2.4 Gazetteer entries (a) from original gazetteer (Paynter and Traylor, $1991 \mathrm{~b}),(\mathrm{b})$ as digitised

The database holds locality names, states, known geocodes and description. This allows proper management of entries, provides verification mechanism for localities with or without geocode or even those referring to other entries. It also allows mutual consistency checks, for instance, on explorer travels as documented through the gazetteer entries.

\subsection{Spatial hints and their components}

\subsubsection{Spatial hint types}

The gazetteer descriptions contain a number of statements or phrases, which we call as spatial hints as they help in determining the position of the locality. Each gazetteer entry on average contains about three spatial hints. We analysed all the entries from the gazetteer and identified seven frequently occurring spatial hint types. The hints are explained below:

Elevation: Gazetteer entry description often starts with an elevation hint. It is mostly expressed with a mention of unit, either ' $\mathrm{m}$ ' or ' $\mathrm{ft}$ '.

Place name and geocode: This hint type comes in a triplet of place name, its geographic coordinate pair and authorizing organization, and together they represent a reference point. For example in Figure 2.2, 'Teresa Cristina [2448/5107 (USBCN)]' and 'Rio Ivaí [2318/5342 (USBGN)]'. The coordinate pair in latter represents the mouth of that river. Note that the organization is the source from which the coordinate information was obtained. 
Direction part-of region: This hint expresses a containment relation, indicating that a locality is described within some part of a region. The parts are described by standard or non-standard compass directions. The standard compass directions are, for instance, 'southern', or 'southeastern', whereas non-standard directions use a prefix plus a standard compass directions, as in 'central southern' or 'southern central', and 'extreme northern' or 'extreme northeastern'. We also call this hint type - directional containment hint.

Distance direction from a reference point: Hint of this type describes the location of a locality with reference to a distance, compass direction from some point. The distance is mentioned in ' $\mathrm{km}$ ' unit and sometimes in 'mi 1es' as well, whereas for directions any of the 16 compass directions can be mentioned. For example, ' $30 \mathrm{~km} \mathrm{NE}$ of city of Rio de Janeiro [2254/4314 (USBGN)]' as shown in Figure 2.4(a).

Distance direction along/from a reference line: In this hint type, locality is described along a line feature, which could be a river, road or railroad. For a river case, a reference point is either its mouth or a locality situated close to river is mentioned and the distance is measured from it, whereas the direction is always along the line using words 'above' or 'upstream' (origination point of river) and 'below' or 'downstream' (mouth of the river). For example in Figure 2.5, 'AIRÃO' is described to be 'on right side of lower middle Rio Negro, at mouth of Rio Jaú' and ' $200 \mathrm{~km}$ above Manaus'. So the distance is mentioned from the point on river close to 'Manaus' and the direction 'above' is along 'Rio Negro' towards its origination point.

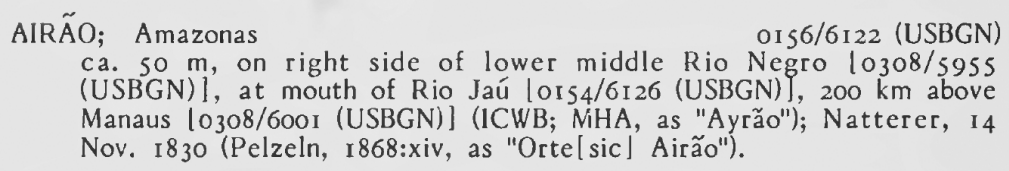

Figure 2.5 An example of a distance direction along/from a reference line hint (Paynter and Traylor, 1991a)

Between two features: This hint type describes a locality as between two spatial features. The two features can be a point-point, line-line, point-line, point-polygon and line-polygon combinations. Generally, a point can refer to a city, a river mouth, or some important structure, whereas a line can be a river, highway, railroad, coast line or state boundary. Examples are, 'halfway between Laje [1310/3925 (USBGN)] and Nazaré [1302/3900 (USBGN)]' or 'between Rio Araguaia [0521/4841 (USBGN)] and Braço Menor do Rio Araguaia [0950/5012 (USBGN)]'. Among all other hints, this is one of the most complex hints to interpret. 
Distance offset: This hint represents a distance of a locality from a reference point, but with no reference to compass direction associated with it. The distance in this hint can be expressed either quantitatively or qualitatively. For example, ' $25 \mathrm{~km}$ from Curitiba [2525/4915 (USBCN)]' or 'near town of Rio Pomba [2117/4311 (USBGN) ]'.

Direction offset: This type of hint describes a locality from a reference point in a specific direction, but with no mention of distance from the reference point. For example, 'S of Franca [2032/4724 (USBGN)]' or 'NNE of Belém [0127/4829 (USBGN)]'.

These spatial hints can further be put under two categories. One that allows to compute location of a locality and another that may only be used for verification after the computation is done. We believe that place name and geocode, distance direction from a reference point, distance direction along/from a reference line, between two features can be categorised as computation hints and elevation, direction part-of region, distance offset, direction offset can be categorised as verification hints.

\subsubsection{Components of spatial hints}

Typically, spatial hints contain information that is useful in finding the locality. In these hints, the lesser known places are described with reference to well-known places. For example, in Figure 2.6, location of 'ÁGUA FRIA' is described with reference to well-known place 'Cuiabá' indicating distance and direction relations, besides being located in 'central Mato Grosso'.

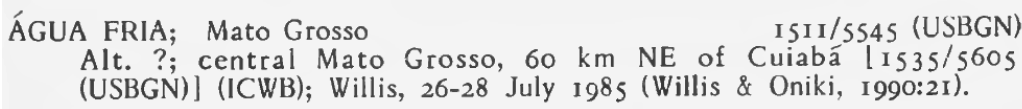

Figure 2.6 An example entry describing lesser known place 'ÁGUA FRIA' with reference to well-known place Cuiabá (Paynter and Traylor, 1991a)

Liu et al. (2009) mentioned when finding the location of a locality using spatial hints, three components should be considered, i.e., the reference object, target object and the spatial relation. We suggest that there should be two components of spatial hints, i.e, spatial features and spatial relations. Here the reference object and target object are spatial features, whereas relations, such as distance, direction and alike can be treated as spatial relations.

A spatial feature can be a topographic or man made entity on the earth's surface that can be mapped, e.g., a river, lake, bridge, island, city, building, road. In our gazetteer, spatial feature is often mentioned with its coordinate pair, e.g., 'Cui abá [1535/5605 (USBGN)]' in Figure 2.6. When the reference feature is a river then coordinate pair of the river 
mouth is provided. From a GIS perspective a spatial feature, could be a point, line or an area feature.

A spatial relation expresses how some features are placed in relation to other features in space. A spatial relationship should be shared between at least two spatial features. In natural language, identification and understanding of spatial relations is important, since these are considered to be the building blocks of geospatial semantics (Arpinar et al., 2006). These spatial relations can provide effective support to various spatial reasoning tasks. There are typically three types of spatial relations:

- Topological relations, describing properties such as adjacency and containment. The adjacency relation can exist between two regions (which are commonly represented as polygons) that share a common boundary. If the two regions are states or countries then the common boundary between them is a border. For example, 'border between Bahia and Minas Gerais' in the text of Figure 2.7.

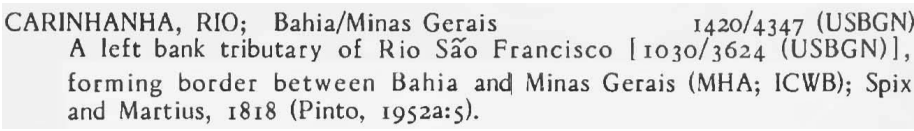

Figure 2.7 Example entry for adjacency relation (Paynter and Traylor, 1991a)

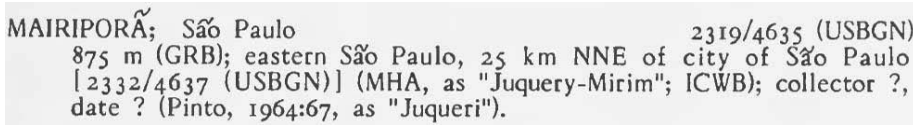

Figure 2.8 Example entry for containment relation (Paynter and Traylor, 1991b)

Containment relations express how one feature is placed inside another feature. For example, a point can be inside some region which can be inside some other bigger region, i.e., a town in a municipality and a municipality in a state. For example in Figure 2.8, a city of 'MAIRIPORÃ' is described to be located ' in eastern São Pau1o' state.

- Direction relations describe the location of the SF using direction relative to some other SF. There can be two types of direction relation: compass direction and relative direction. There are 16 compass direction of which four are cardinal directions: $\mathrm{E}, \mathrm{W}, \mathrm{N}, \mathrm{S}$, another four are ordinal directions: $\mathrm{NE}, \mathrm{NW}, \mathrm{SE}$, and SW and there are eight interordinal directions: NNE, ENE, NNW, WNW, SSE, ESE, SSW, WSW. The directions $\mathrm{N}$ and $\mathrm{S}$ represent true magnetic north and south on the Earth.

Relative directions are qualitative in nature, which means that they do not represent any true compass direction, and are mainly relative to the orientation of the observer. In natural language, 


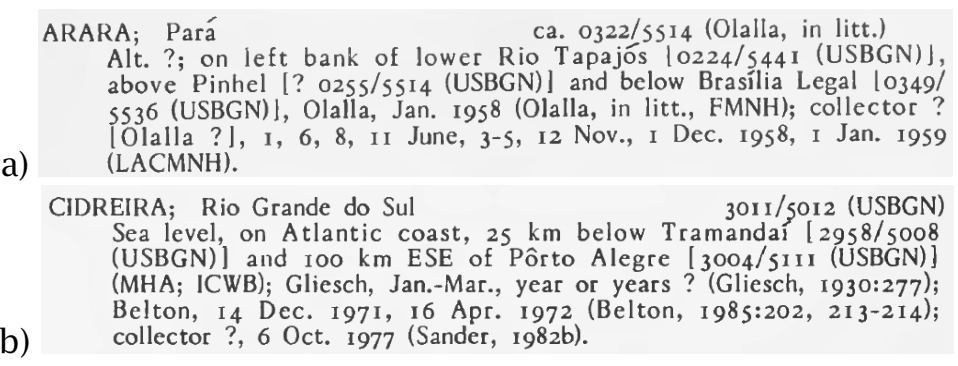

Figure 2.9 Above and below direction relation in connection with (a) river, (b) administrative features (Paynter and Traylor, 1991 a)

they occur as words above or below, 1eft or right. These words should be interpreted in connection with the spatial feature under consideration. In our gazetteer also, the words above and below are used but mainly in connection with two kinds of spatial feature, namely with linear features (river or canal, but not road or rail) as shown in Figure 2.9(a), and with some administrative features (city or state), as shown in Figure 2.9(b).

In Figure 2.9(a), the description says 'on 1eft bank of 1ower Rio Tapajós [0224/5441 (USBGN)], above Pinhe1 [? 0255$/ 5514$ (USBCN)] and below Brasília Legal [0349/5536 (USBGN)]' which means that locality 'ARARA' is somewere on the left bank of lower Rio Tapajós. Here 'above Pinhe1' refers to the direction along Rio Tapajós towards its origination point, whereas 'below Brasília Legal' also refers to direction along the river but towards its mouth. Similarly in Figure 2.9(b), hint ' $25 \mathrm{~km}$ below Tramandaí' refers to the 'south' direction from 'Tramandaí' along the coast line.

However, there are other words such as upper, lower, and middle, that are only used in connection with river-like features in which, upper is mostly that part of the river which is closer to the river head and lower is that part which is closer to the river mouth.

- Distance Relations can be expressed in two ways, quantitatively (by metric measurement) and qualitatively. Quantitative distance represents absolute measurement, in which a value and its unit of measurement is known, such as in '20 km' or ' $26 \mathrm{mi}$ ', whereas qualitative distance does not provide absolute measurement and is often expressed using words such as 'close to, near to' or 'far from', which are easier to express and understand by people but difficult to formalize in computations.

\subsection{Dataset used}

For our base data state boundaries, river network, road network and elevation, data was needed. We obtained Brazilian state boundaries from 
Esri's ArcWorld Supplement Map data, dated 1998.We merged the states of Goiás, Tocantins and the Distrito Federal as a single state 'Pre-1988 Goiás' to reflect the gazetteer's notion of Goiás state. The data for roads, rail roads and river network was also obtained for the year 2001 from the Instituto Brasileiro de Geografia e Estatística (IBGE). We found that for many rivers the line strings were present but with no river names attached to it. To complete our river dataset we obtained missing river data from additional sources, namely Natural earth ${ }^{5}$. We also used road atlas for Brazil (Guia Estradas) to manually identify the rivers in many states which were later on added to our river database. All our data is stored in geographic latitude/longitude having WGS84 as its datum.

\subsection{Tools used}

In addition to the datasets, we used following tools:

PostgreSQL with PostGIS Popularly known as Postgres, it is an objectrelational database management system (ORDBMS). PostGIS is a spatial extension to Postgres for management and analysis of spatial data. Both are free and open source softwares. In this research project, Postgres version 9.1 and PostGIS version 2.0 were used.

Quantum GIS This tool was used to visualise base map data and the interpretations models that were created. It is an open source GIS software, and version 1.7.4 has been used in this project.

ILWIS This tool was used to visualise probability rasters created for different spatial hint types. It is an open source software, and version 3.8 has been used in this project.

Stanford Parser It is a probabilistic natural language parser from Stanford Universitys' NLP group. This parser works on the grammatical structure of the input sentences and produces a tree structure for them. For this research project, a Java implementation of parser version 1.6.5 is used, licensed under the GNU.

Stanford Tregex and Tsurgeon This tool was used for matching patterns in parse trees based on structure and regular expression matches in the tree. The Java implementation of this tool was used in conjunction with parser to extract information from parse trees. Version 1.4.1, licensed under GNU, has been used in this research project.

SPSS Statistics This software was used to carry out various statistical analysis in this project and Version 21 was used.

\subsection{Summary}

We work with ornithological gazetteer of Brazil written by Paynter and Traylor (1991a,b) containing 8,000 entries covering roughly 6,000 sites.

\footnotetext{
${ }^{5} \mathrm{http}: / /$ www.naturalearthdata.com/
} 
This gazetteer is interesting to work on because it provides semi-structuted textual descriptions that may or may not contain geocodes. In the process of understanding gazetteer entries we found that a typical entry contains place name, state name, geocode (if mentioned) and a description. Each description further carries various spatial hints in the form of elevation, place name, reference features, distance and direction among others. These spatial hints carry important information that needs to be extracted for geocoding of localities. The extraction of such information is discussed in Chapter 3. 



\section{Extracting spatial hint information from locality descriptions}

\subsection{Introduction}

As discussed in Section 2.2, a gazetteer entry contains place name, state name, geocode and a description. The description contains spatial hints that describe a locality with reference to other features. For example, in Figure 3.1, the location of 'MANTENÓPOLIS' in the state of 'Espí rito Santo' is described using four spatial hints, i.e., elevation, directional part-of region, nearby features and distance direction from a reference point.

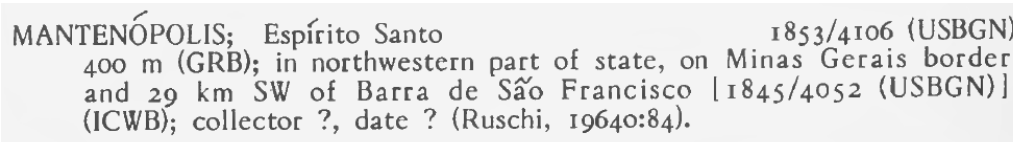

Figure 3.1 An example entry from the gazetteer (Paynter and Traylor, 1991b)

These hints contain important information that can be used to geocode the locality, or verify the provided location. In above example, value for elevation hint is ' 400 ' with unit ' $m$ ', the directional part-of region hint says 'in northwestern part of state', which is important. Likewise, different hints contain different information. The hints are easier to understand by humans but are difficult to be exploited by machine, yet still allow for various spatial analyses or computations.

We believe that before any spatial computation can be realized, an extraction of spatial information from these hints and storage into data structures entities is required. This falls in the discipline of information extraction through Natural Language Processing (NLP). In this, a natural language description is parsed through a text processing tool, and further, hint-specific information is extracted and stored in a structured form. This process is the main focus of this chapter.

The sections in the chapter are arranged as follows. A literature review on information extraction (IE) from a spatial information perspective is provided in Section 3.2. Section 3.3 discusses our approach 
and implementation of information extraction. From Section 3.4 to Section 3.7 various steps followed during information extraction are discussed. Finally, Section 3.8 discusses evaluation of developed patterns and extraction results, followed by a summarising section.

\subsection{Related work}

Information Extraction is a process of extracting domain-specific structured information from natural language text (Moens, 2006). This structured information can be about subject of interest, relationship between subjects and attributes describing subjects. The interest in information extraction started in late 1980's and early 90's with the Message Understanding Conference (MUC). In each MUC, participating groups developed their own systems to process text messages. These groups were then given sample messages and were instructed to extract specific information (Grishman and Sundheim, 1996). MUC-3, was dedicated to detection and extraction information from text containing terrorist events (Grishman and Sundheim, 1996). Information, such as type of attack, perpetrator, physical target, human target, instrument and location of incident (Jackson and Schilder, 2006) were to be extracted by the participating groups' systems. Named Entity Recognition (NER) was first mentioned in 1996 in MUC-6 conference (Grishman and Sundheim, 1996) in which the goals of the conference was to encourage the development of information extraction systems for practical uses. The other subtask in MUC- 6 was to develop "named entities" that can be used to identify person names, organisations, places, time, currency and percentage expressions (Grishman and Sundheim, 1996).

Following MUC series, in 1998 another conference, Automatic Content Extraction (ACE) was started with the focus on recognizing entities, entity relation and event extraction from text and had listed 100 different entity types (NIST, 1998). Later many applications were developed to identify entities such as persons, locations and organizations from text documents. Some of the early systems of information extraction systems were FASTUS (Appelt et al., 1993), Learning Information Extraction Patterns (LIEP) (Huffman, 1995), Parallel Automatic Linguistic Knowledge Acquisition (PALKA) (Kim and Moldovan, 1995), AuloSlog (Riloff, 1996) and many others. Rewiew of these systems are provided in (Muslea, 1999; Zhou and Zhou, 2004).

Early information extraction systems were rule-based that required manually coded rules. The rule-based technique is used where the extraction task is controlled and well-behaved, such as in extracting phone numbers, place names, addresses or where annotated training corpus is not available (Sekine and Nobata, 2004). There are various languages for the rule-based technique such as Java Annotation Pattern Engine (JAPE), regular expression patterns, datalog (Sarawagi, 2008). This technique makes use of various features such as capitalization, token type, orthography type, pos tags and other features depending on the type 
of data available and the type of extraction task to be carried out. As manual coding of rules can be very tedious, algorithms were developed for automating this task. With time, models based on statistical techniques such as Hidden Markov Models (HMM) and maximum entropy models were introduced (Sarawagi, 2008). Both of these were superseded by Conditional Random Fields (CRF), as they needed large amount of training datasets (Feldman, 2006). In the current times, however both rule-based and statistical-based models are used depending on the type of the extraction tasks. There are also some hybrid models that use both methods (Sarawagi, 2008).

Applications of information extraction are most widely found in biomedical domain especially in genetics. For example, in GENIA (Collier et al., 1999) project which aims to construct database of human genome by extracting biochemical information from journal papers. In project MedLEE (Friedman, 1997), NLP based extraction system was developed to extract specific information from radiology reports. In (Hagège et al., 2002), authors have presented an approach on detection of entities in genetic text using special dictionary and context-based rules to extract gene names not present in the dictionary. Napolitano et al. (2010) used pattern based approach to extract information from free-text pathology reports.

In geographic information domain, geoparsing is a form of NER and is also known as Geographic Entity Extraction (Fu and Sun, 2011). The main task of geoparsing is to process natural language text in order to identify and extract geographic entities, disambiguate them and assign geographic coordinates. Place names are the most commonly occurring geographic entities in text documents (Fu and Sun, 2011). Commercial software applications, such as OpenCalais, MetaCarta, Yahoo! Placemaker, GeoLocator and web services, all perform geoparsing. Geoparsing can actually be quite a difficult task since identification and disambiguation of place names in text depends largely on the use of language and its interpretation by the software applications (Abascal-Mena and López-Ornelas, 2010). Almost all commercial applications use existing place name gazetteers, such as Geonames, ${ }^{1}$ or $\mathrm{ADL}^{2}$ for place name recognition. When used over historical text, they may not give good results because the existing gazetteers may not contain old place names or variation in spellings of those names.

Research has also been done in extracting information from route descriptions, travelogues, for depicting places or reconstructing travel paths. To extract route parts, i.e., origins and destinations from human generated route directions, Zhang et al. (2010) used rule-based and machine learning techniques. First, they used regular expressions to extract relevant features and then those features are used to train four different machine learning models, which were then evaluated to find the best model. However, in their technique, no actual routes were reconstruc-

\footnotetext{
${ }^{1}$ www.geonames.org

${ }^{2}$ www.alexandria.ucsb.edu
} 
ted. In (Drymonas and Pfoser, 2010), a methodology was described for reconstructing travel paths by extracting landmarks along the routes from travel diaries. In their approach, rule-based IE engine is used in conjunction with location ontology. The rule-based engine extracts place names, whereas spatial ontology is used in describing the spatial relationships between the extracted places. Rule-based approach and statistical approach were used by Habib and van Keulen (2011) to extract place names and disambiguate place names from text descriptions. Beaman et al. (2004) used semi-automated approach using existing gazetteer and NLP tools to interpret locality descriptions. In (Godoy et al., 2011), lexico-syntactic patterns and co-reference analysis were used to extract place names in text document with one of the purposes being expanding the gazetteer with new geographic locations. Leidner et al. (2003) used NER based approach to extract place names from newswire text with the purpose of mapping them automatically to see where the reported news event had taken place. Gelernter and Mushegian (2011) used rule-based NER to extract place names from twitter microtexts.

Adeva (2008) used web service that provides standard locations for vernacular terms that were extracted using statistical approach. Batista et al. (2010) used machine learning approach to extract geographic entities from text documents. Yamamoto et al. (2011) presents an approach to extract historical events from the web to geographically navigate through them.

As can be seen, depending upon the project requirement, people have used both rule-based and statistical methods in extracting information from text documents. Statistical methods are used where annotated corpus for training the parsers or entity recogniser are available, whereas rule-based methods were recommended are there is no availability of annotated corpus for training parsers or entity extraction tools (Sekine and Nobata, 2004). In the next section, we will discuss the approach we took to extract information from our gazetteer corpus.

\subsection{Approach and implementation}

\subsubsection{Approach}

In our approach, a Natural Language Processing (NLP) based technique is used for extraction of various information from our gazetteer corpus. In NLP, a free form text is analyzed syntactically, to determine the grammatical structure of a sentence. The grammatical structure provides information about the phrasal structure present in the sentence. A typical NLP system has four components: sentence splitting, tokenization, PartOf-Speech (POS) tagging and syntactic analysis and parsing as shown under 'NLP pipeline' in Figure 3.2. Same figure also shows broadly the methodology we adopted for implementation of the whole extraction task. 
We also considered using NER (Named Entity Recognition), however, we did not find NER suitable for our work because it only helps in recognition of entities such as place name, persons' names and like. It does not help in complete analysis of the sentence structure that can give us important information on the distance, direction and others mentioned in each description in our gazetteer. NER models can be trained to extract required information, for which however, they require annotated corpus which we do not have. Hence we based our information extraction task over NLP technique. In the following section, we discuss the implementation of extraction task in detail by explaining our steps.

\subsubsection{Implementation}

For our implementation, we make use of existing technology from the Natural Language Processing (NLP) and Information Extraction (IE) domains. The implementation can be explained in five steps, as marked in Figure 3.2.

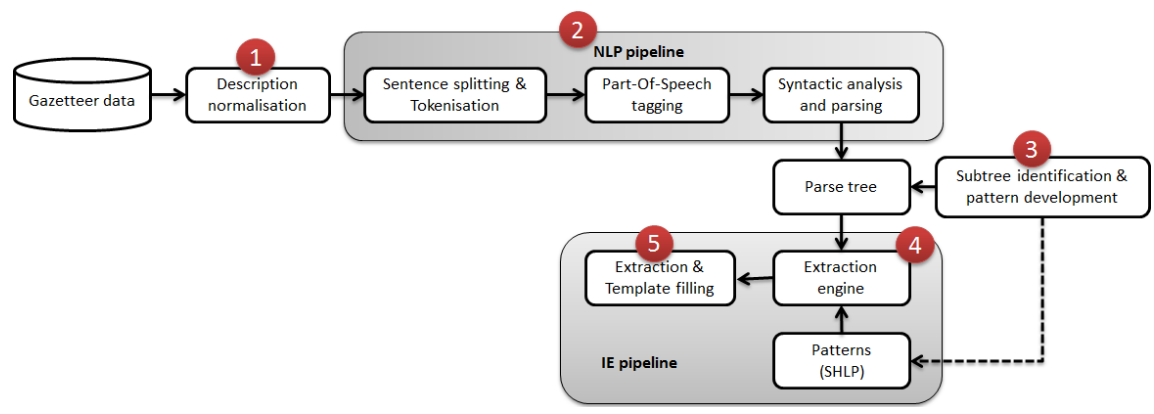

Figure 3.2 The complete extraction pipeline implementation

1. In the first step, we identify and normalise the descriptions in the database before they are sent to the NLP pipeline. During normalisation, we remove any unwanted symbols such as extra period, we rectify abbreviations and compound names, which would otherwise lead to erratic parser behavior. The list of problematic elements is given in Table 3.1.

2. In the second step, normalised descriptions are passed on to the NLP pipeline, where description goes through various language processing steps for further syntactic analysis, after which a parse tree structure of the description is generated.

3. In the third step, we identify various subtrees in the parse trees and develop patterns for extraction of information. The patterns are developed manually using regular expressions specifically designed for tree structures (Levy and Andrew, 2006).

4. In this step, the extraction engine uses two inputs, the first being a parse tree and the second being a set of extraction patterns. The 
extraction engine searches for patterns that match parts of the parse tree.

5. A simple hint extraction algorithm subsequently gathers the matched pattern information from spatial hints in the parse tree, and inserts them in a structured form in a hint template in the database.

The following sections provide a detailed discussion of steps in our implementation.

\subsection{Description normalisation}

This is the first step in extraction process as also shown in Figure 3.2. The textual descriptions stored in the database contain problematic elements that potentially confuse the tagger and lead to assignation of wrong tags during the parse process. The reason is that some of those words do not appear in the parser training data. To overcome this problem, we identified those elements (Table 3.1) that must be rectified first, before the description is passed to the parser. These elements are the presence of some symbols, abbreviations, redundant information and compound names.

Table 3.1 Problematic elements present in gazetteer entries

\begin{tabular}{|c|l|l|}
\hline No. & Problematic elements & Simplified elements \\
\hline \hline 1. &. . or $?$ & Unknown \\
\hline 2. & $=$ & Removed from the description \\
\hline 3. & Ca. or ca. or ca & Circa or circa \\
\hline 4. & Jan., Feb., ... & January, February \\
\hline 5. & ne, se, sw, ... & $\begin{array}{l}\text { northeastern, southeastern, south- } \\
\text { western }\end{array}$ \\
\hline 6. & e, w, n, s & $\begin{array}{l}\text { eastern, western, northern, sou- } \\
\text { thern }\end{array}$ \\
\hline 7. & município [county] & municipality \\
\hline
\end{tabular}

To rectify or improve all the problematic elements together, we developed a function that performs the required replacements. For instance, if the original description is:

' $548 \mathrm{~m}$, town in se part of state, $47 \mathrm{~km} \mathrm{NE}$ of Ponte Nova [2024/4254 (USBGN)] (MHA; ICWB); Erickson, 19 Sept. 1966 (Erickson, 1982:138)',

then after the function execution, some text strings (underlined) in the description are fixed.

Subsequently, for the parsing task to work effectively, all place names should be recognized as a single unit to be tagged properly. In our corpus, a place name can be as long as three to five words e.g., 'Rio de Janeiro' and 'Braço Su1 do Rio Jucurucú'. These place names are identified as separate tokens during a tokenisation process, which further 
splits the place name into different words instead of one composite word. For example, Figure 3.3 shows how the 'Braço Sul do Rio Jucurucú' would be parsed under default settings if used without normalisation, and what we actually desired.
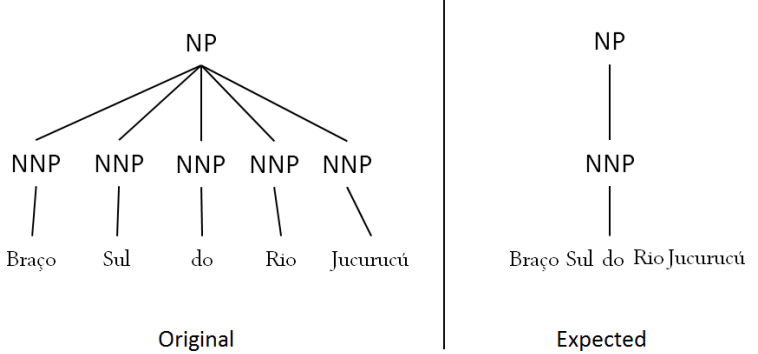

Figure 3.3 Original and expected recognition of compound place name

To recognise such place names as one entity, a small function is developed that uses the Java regular expression patterns to identify them in input string (i.e., description). The function uses three classes, i.e., string, pattern and matcher. The notations used in developing the pattern to identify compound names are ' $W$ ' which recognises any uppercase letter, including accented letters, such as Ã, Ó, Ç, Î and many others. The notation ' $w$ ' recognises any lowercase letter, including accented letters. Notation ' $S$ ' recognises space(s) between the words and notation 'D' recognises the variant of middle words ('de', 'da', 'do', 'das' and 'dos') used in place names. The two developed patterns as shown below run iteratively to find the requisite place name match in the description.

Pattern 1: "Ww+(S+Ww+)*(S+DS+)(Ww+)(S+Ww+)*"

Pattern 2: "Ww+(S+Ww+)+"

Figure 3.4, illustrates which part of the pattern recognises place name words in the description.

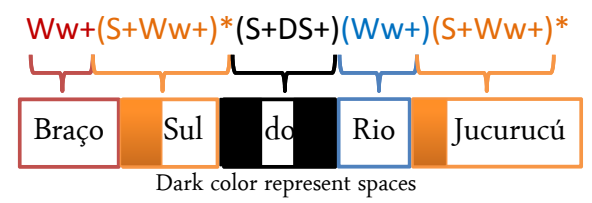

Figure 3.4 Place name recognition through a pattern

By using our function, the place name in the same description mentioned earlier will be changed from 'Ponte Nova' to 'Ponte_Nova'.

The descriptions are now ready to be processed by the NLP pipeline, which is step-2 in Figure 3.2 and is described in the following section. 


\subsection{Natural Language Processing pipeline}

This is the second step of extraction process as shown in Figure 3.2. Natural language processing (NLP) is a subfield of artificial intelligence and linguistics, with a goal of studying natural language text for information retrieval, information extraction and summarisation, among others. As mentioned before, NLP allows syntactic analysis of a free-form text, to determine the grammatical structure of a sentence. The four components of a typical NLP system as shown in Figure 3.2, are explained in following sections.

\subsubsection{Sentence splitting and Tokenisation}

In sentence splitting, we split the text based on a predefined set of delimiters such as commas, dots and colons into sentences. Tokenisation breaks the textual unit ${ }^{3}$ in the sentence, into a list of simple tokens, such as words, numbers, and punctuation symbols. The ordering of these two tasks does not matter, i.e., tokenisation can be performed before or after sentence splitting.

\subsubsection{Part-Of-Speech tagging}

In this step, tokens are assigned with linguistic metadata. This metadata comes from a treebank which is syntactically processed and consists of labels (a.k.a. Part-Of-Speech tags) of natural language data. These labels are then utilised at various linguistic levels, such as word, phrase, clause and sentence level (Moens, 2006).

Table 3.2 Penn Treebank Part-Of-Speech tags (taken from (Bies et al., 1995))

\begin{tabular}{|c|l|l|}
\hline No. & Tag & Description \\
\hline 1 & CC & Coordinating conjunction \\
\hline 2 & CD & Cardinal number \\
\hline 3 & DT & Determiner \\
\hline 4 & IN & Preposition or subordinating conjunction \\
\hline 5 & JJ & Adjective \\
\hline 6 & NN & Noun, singular or mass \\
\hline 7 & NNS & Noun, plural \\
\hline 8 & NNP & Proper noun, singular \\
\hline 9 & RB & Adverb \\
\hline 10 & SYM & Symbol \\
\hline 11 & VB & Verb, base form \\
\hline
\end{tabular}

Traditionally, in English, there are eight main POS tags, i.e., noun, verb, adverb, adjective, preposition, determiner, conjunction and interjection (Wilcock). Each of them have been further divided into subcategories. For example, a noun can be singular noun or plural noun. In English,

\footnotetext{
${ }^{3}$ Each word, number or a punctuation in a text string is referred to as a textual unit.
} 
the most commonly used treebank corpus is the Penn Treebank ${ }^{4}$, which consist of 36 POS tags few of them are shown in Table 3.2 and Appendix A shows the complete list of Penn Treebank II tag set at clause, phrase and word levels along with punctuation tags.

\subsubsection{Syntactic analysis and parsing}

The task of syntactic analysis is to analyze the POS tags and assign them to a higher linguistic category following some syntactic rules (a.k.a. syntactic structures). The higher linguistic category corresponds to a phrasal structure in a sentence, such as Noun Phrase (NP), Verb Phrase (VP), or Prepositional Phrase (PP). In Penn treebank, there are 21 different phrase tags as shown in Table A.2 (Appendix A) under which the words can be grouped to form a sentence phrase. In the following sections, standard syntactic rules and context-free grammar are discussed.

\subsubsection{Syntactic rules}

The syntactic rules specify which word sequences are acceptable in a sentence and how they should be grouped together to form nodes in a parse tree. The syntactic rules consist of POS tags and production symbol $(\rightarrow)$ which should be interpreted as 'can take the form of'. Let us consider some basic rules represented as expressions below:

$$
\begin{aligned}
& S \rightarrow N P V P \\
& N P \rightarrow D T \text { noun } \\
& V P \rightarrow \text { verb } N P \\
& V P \rightarrow \text { verb }
\end{aligned}
$$

In the LHS side, only one non-terminal node is allowed, whereas on the RHS non-terminal as well as terminal nodes can exist. Non-terminal nodes are those nodes under which other nodes can exist but not words, numbers, punctuations or symbols. Terminal nodes are those nodes that can not have other nodes under it except for words, numbers, punctuations, or symbols under it. A terminal node can never occur on the LHS of the rule. The symbol $S$ represents the start symbol of the syntax. The symbol NP represents a noun phrase, which contains a group of words that name(s) a thing(s) and can be used as a subject or an object. For example, a noun in RHS of the rule above can be represented as noun $\rightarrow$ 'Enschede'. Similarly, the symbol VP represents a verb phrase, which contains action words that can be tagged as ver $b$, like in ver $b \rightarrow$ 'moving' | 'going' | 'entering', and a determiner (DT) can take the form of the word 'a' or 'the' represented as DT $\rightarrow$ ' $a$ ' | 'the'.

These rules are an important part of building the parser grammar. One type of grammar that uses those expressions or syntactic rules is a context-free grammar. It is discussed in the following section.

\footnotetext{
${ }^{4}$ Penn Treebank POS tags, http://www.cis.upenn.edu/ treebank/
} 


\subsubsection{Context-Free Grammar}

Context-Free Grammar (CFG) is a fundamental formalism when parsing algorithms are described (Aho and Ullman, 1972). A CFG uses basic syntax rules as detailed in Section 3.5.3.1. The formal definition as taken from (Aho and Ullman, 1972) states that a CFG (represented by symbol $\mathrm{G}$ ) is a quadruple defined as $\mathbf{G}=[\mathbf{N}, \Sigma, \mathbf{R}, \mathbf{S}]$. The symbol $\mathbf{N}$ represents a finite set of non-terminal nodes (such as NP and VP) and symbol $\Sigma$ represent a finite set of terminal nodes; the two are disjoint. $\mathbf{S}$ is the start symbol representing the sentence, such that, $\mathbf{S} \in \mathbf{N}$. The symbol $\mathbf{R}$ represents a finite set of syntax rules of the form $\mathbf{X} \rightarrow \mathbf{Y}$, where $X \in N$ and $Y \in(\Sigma \cup N)$.

According to the definition in (Aho and Ullman, 1972), a labeled ordered tree $\mathbf{P}$ is a parse tree for a context-free grammar $\mathbf{G}(\mathbf{S})=[\mathbf{N}, \Sigma, \mathbf{R}$, S] if,

1. The root of $\mathbf{P}$ is labeled as $\mathbf{S}$.

2. If $\mathbf{P}_{1}, \ldots, \mathbf{P}_{k}$ are the subtrees of the direct descendants of the root and the root of $\mathbf{P}_{1}$ is labeled $\mathbf{X}_{1}$, then $\mathbf{S} \rightarrow \mathbf{X}_{1}, \ldots, \mathbf{X}_{k}$ is a production in $\mathbf{R}$. $\mathbf{P}_{1}$ must be a parse tree for $\mathbf{G}\left(\mathbf{X}_{1}\right)=\left[\mathbf{N}, \Sigma, \mathbf{R}, \mathbf{X}_{1}\right]$ if $\mathbf{X}_{1}$ is a non-terminal, and $\mathbf{P}_{1}$ is a single node labeled $\mathbf{X}_{1}$ if $\mathbf{X}_{1}$ is a terminal.

3. Alternatively, if $\mathbf{P}_{1}$ is the only subtree of the root $\mathbf{P}$ and the root of $\mathbf{P}_{1}$ is labeled $\boldsymbol{e}$, then $\mathbf{S} \rightarrow \boldsymbol{e}$ is a production in $\mathbf{P}$.

Many parsing algorithms work with the formalism of CFG and some use the probabilistic formalism of CFG (a.k.a. PCFG).

In PCFG, every syntax rule is associated with a measure of probability (Booth and Thompson, 1973). The probability represents the measure of likelihood of rule application being in the derivation of a parse tree.

A PCFG can be formalized as an quintuple: $\mathbf{G}=[\mathbf{N}, \Sigma, \mathbf{R}, \mathbf{S}, \mathbf{D}]$ (Ljunglöf and Wirén, 2010). The terminology remains same as in the CFG, except for $\mathrm{D}$, which is a function that assigns probability to each member of $\mathrm{R}$ such that, D:R $\rightarrow[0,1]$.

According to (Ljunglöf and Wirén, 2010), let L(G) denote the string language generated by $G$ and $T(G)$ denote the tree language generated by $\mathrm{G}$ in such a way that $\mathrm{T}(\mathrm{G})$ is a set of parse trees corresponding to valid derivations of strings in $\mathrm{L}(\mathrm{G})$. For any parse tree $y$ in $\mathrm{T}(\mathrm{G})$, YIELD(y) denotes the product of probabilities of all rules in the parse tree $y$. Likewise, COUNT(i, y) denotes the number of times any syntax rule used in the parse tree.

YIELD $(y)=$ yield for the terminal string such that $y \in T(G)$. COUNT(i, $\mathrm{y})=$ the number of times that the $\mathrm{i}^{\mathrm{th}}$ syntax rule $\mathrm{r}_{i} \in \mathrm{R}$ is used in the derivation of $y$.

Given a parse tree $y \in T(G)$ the probability of a parse tree is calculated by multiplying the probability of its individual subtrees:

$$
P(y)=\prod_{i=1}^{|R|} D\left(r_{i}\right)^{\operatorname{COUN} T(i, y)}
$$


The above equation is based on basic probability theory, in which the assumption is that the syntax rule in the derivation of a tree is independent of other syntax rules in the tree.

In the following section we discuss parsing of the descriptions.

\subsubsection{Parsing descriptions}

Given a sentence, the task of parsing is to produce a syntax tree (a.k.a. parser tree) adhering to some standard grammar. Parsing is an important step in information extraction from natural language descriptions, since the generated parse tree serves as an input to the information extraction pipeline and is a building block for the development of our patterns.

In this project, we used the Stanford parser (hereafter: parser), which is a probabilistic parser and uses a PCFG grammar for parsing our locality descriptions. We used PCFG because CFG is not expressive enough and cannot describe all peculiarities of natural language (Ljunglöf and Wirén, 2010). We established the database connection with the parser so that input descriptions from the database can be passed to the parser to produce a parse tree, after it has passed through the NLP pipeline. The descriptions that are passed to the parser have been normalised (Section 3.4). The output parse tree from one such description ' $548 \mathrm{~m}$, town in southeastern part of state, $47 \mathrm{~km} \mathrm{NE}$ of Ponte Nova [2024/4254 (USBGN)] (MHA; ICWB); Erickson, 19 Sept. 1966 (Erickso$n, 1982: 138)$ ' is shown in Figure 3.5.

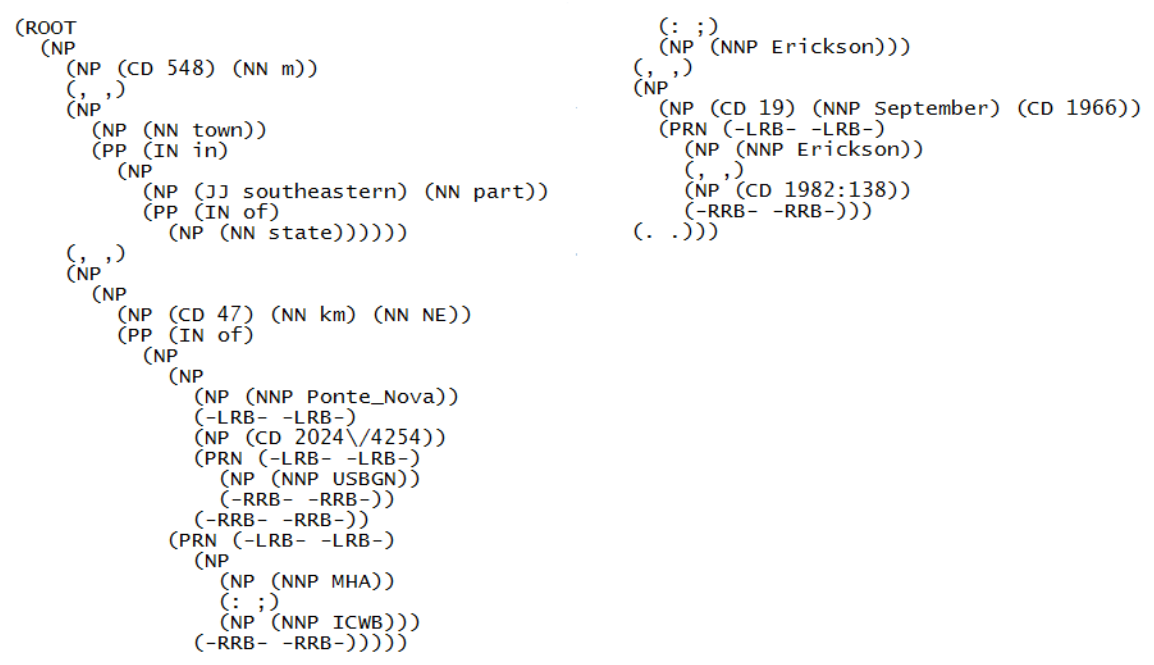

Figure 3.5 Output from the Stanford parser for a description

This parse tree shows how textual units are logically connected to others, following the probabilistic rules. The words are assigned POS tags, which are terminal nodes and these terminal nodes are grouped together to form non-terminal nodes such as NP, PP and PRN. The non- 
terminal nodes tagged with PRN show the presence of parentheses with its terminal nodes as "-LRB-" and "-RRB-."

After analyzing the parse tree, we find that place names can be found as NNP terminal nodes, under a NP node. Observe that nodes tagged as noun are not always place names, as can be seen in the parse tree, in which distance unit $(\mathrm{km})$ and compass direction (NE) are also tagged as noun variant. Likewise, a tree may contain same structure more than once but with different information. Identification of such information is important to unearth new spatial information from printed gazetteers. In the following section, we discuss subtree identification in parse trees and pattern development for extraction. This forms our third step in Figure 3.2.

\subsection{Identifying subtree patterns in parse trees}

An important step in IE is to identify and extract information units from text segments. In our case, these text segments are spatial hints, and information units are words that needs to be extracted. We generate parse trees for all entry descriptions in our gazetteer. A parse tree provides a coherent analysis of syntactic structure of a description. These parse trees can be used to identify spatial hints by identifying subtrees. Often in our descriptions, place name is followed by its geocode and naming organization, and also, distance information is followed by direction information.

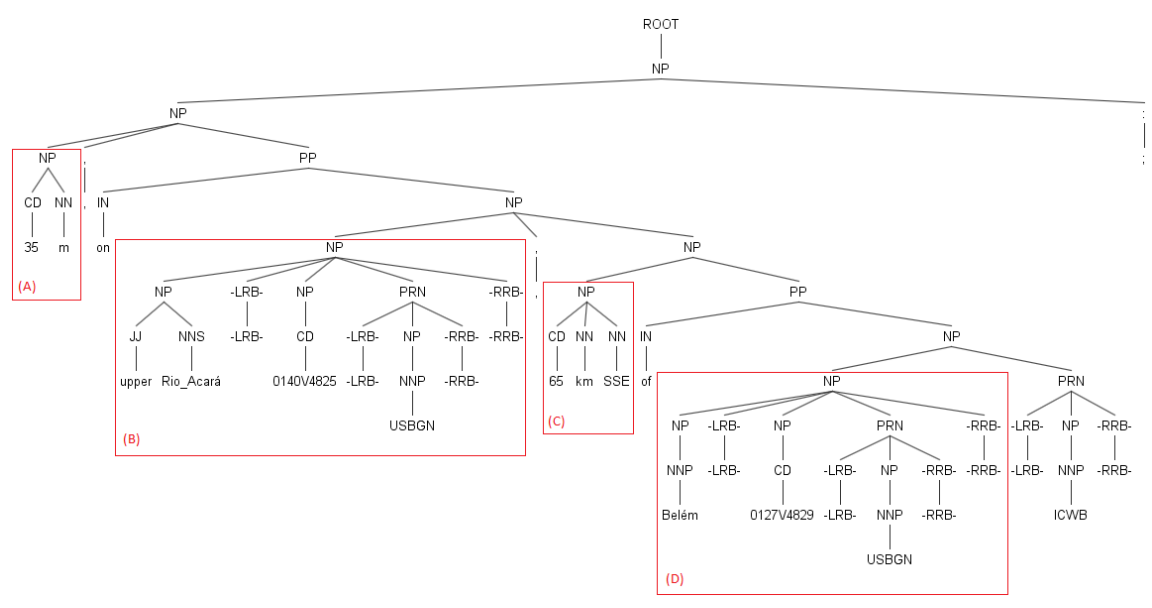

Figure 3.6 Example of four subtrees identified in a parse tree

For example, for description ' $35 \mathrm{~m}$, on upper Rio Acará [0140/4825 (USBGN)], $65 \mathrm{~km}$ SSE of Belém [0127/4829 (USBGN)] (I(WB); 01a11a, 12 0ct. 10-26 Nov. 1933 (01a11a, 1935:420;Griscom \& Greenway, 1941:92, as "Vi11a Acara," MCZ, as "Vi11a Acar"), col1ector?, 6 Mar. 1944 (Ruschi, 1949e:1); Pin- 
to, 1949b:322, as "Vi 1a Acara"'. Figure 3.6 shows part of the parse tree in which four subtrees can be identified. In that parse tree we identify subtrees for elevation as (A), mention of some part of reference feature and geocode as (B), distance and direction as (C) and reference palcename and geocode as (D). It is important to note that subtrees that look similar in their structure may contain different information. For example, the hint subtree (B) of 'upper Rio Acará [0140/4829 (USBCN)]' is similar to the hint subtree of (D) 'Belém [0127/4829 (USBGN)]', but has an additional information 'upper' tagged as adjective, which tells something about the part of the river. Interestingly, while developing patterns, one or more subtrees can be combined to extract information from a hint. For instance, again in Figure 3.6, the subtree of (C) 'distance and direction' must be combined with that of (D) 'reference placename and geocode' to extract information units for hint type 'distance direction from a reference point/place'.

Further, we identify frequently occurring subtree structures of spatial hints in parse trees of our descriptions in Table 3.3. The LHS (nonterminal node) represents the parent node, whereas the RHS (both terminal and non-terminal nodes) represents nodes that carry information that must be extracted. The nodes in RHS can further be categorised as direct and indirect nodes. The direct nodes carry the information to be extracted, such as NNP, IN, JJ, and indirect nodes are parent nodes to direct nodes, such as NP, PP.

A noun tag is considered as a candidate for place name (Adeva, 2008; Godoy et al., 2011). However, in our descriptions there are words such as 'circa', 'km', ' $m$ ', and others that are tagged as noun, and they do not represent any place name. Also, the noun tag may be for a singular noun (NNS), plural noun (NN) or proper singular noun (NNP). For example, in Table 3.3 subtree structures S3 to S6 operator '|' is used, which means that word may be tagged as either NN or NNP. The reason for this is because there is no trained parser for the geographic text domain, and all existing parsers have been trained on manually tagged corpus of the Wall Street Journal or Brown corpus (from English literature). These corpora do not contain words used in the geographic domain, and hence the parser does not know the lexical relationships between such terms in our descriptions, and may provide wrong tags to them. As mentioned in Section 3.3, to train a parser an annotated corpus is required which is not available for our case and hence training of parser was not done.

In the next section we discuss the language that we have used to develop patterns for extraction of information from gazetteer descriptions.

\subsubsection{Developing patterns for extraction}

Developing patterns is an important step, as it allows us to find information in the parse trees, which can be then extracted and stored in structured format. Hence, a mechanism is needed through which these patterns can be developed so as to make implicit information explicit. For this purpose a tree query language is used, which uses regular ex- 
Table 3.3 Subtree structure of qualification hints in parse trees

\begin{tabular}{|c|c|c|}
\hline No. & $\begin{array}{l}\text { Subtree informa- } \\
\text { tion }\end{array}$ & Subtree structure and example \\
\hline S1 & $\begin{array}{l}\text { Reference place- } \\
\text { name and geocode }\end{array}$ & $\begin{array}{l}\text { NP } \rightarrow \text { NNP CD NNP } \\
\text { Cametá }[0215 / 4930(\text { USBGN)] }\end{array}$ \\
\hline S2 & $\begin{array}{l}\text { Part of reference fea- } \\
\text { ture and geocode }\end{array}$ & 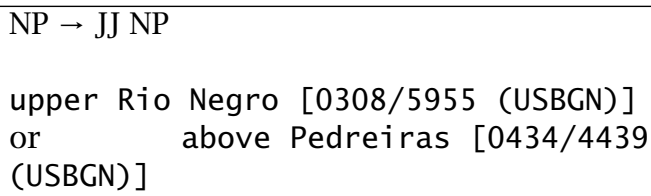 \\
\hline S3 & $\begin{array}{l}\text { Distance and direc- } \\
\text { tion }\end{array}$ & $\begin{array}{l}\mathrm{NP} \rightarrow(\mathrm{CD}(\mathrm{NN} \mid \mathrm{NNP}))(\mathrm{NN} \mid \mathrm{NNP}) \\
14 \mathrm{~km} \text { ENE }\end{array}$ \\
\hline S4 & Elevation & $\begin{array}{l}\mathrm{NP} \rightarrow \mathrm{CD}(\mathrm{NN} \mid \mathrm{NNP}) \\
1,400 \mathrm{~m}\end{array}$ \\
\hline S5 & Approx. elevation & $\begin{array}{l}\mathrm{NP} \rightarrow \mathrm{NNP} \text { CD (NN | NNP) } \\
\text { Circa } 1,400 \mathrm{~m}\end{array}$ \\
\hline S6 & $\begin{array}{l}\text { Approx. distance } \\
\text { and direction }\end{array}$ & $\begin{array}{l}\mathrm{NP} \rightarrow \mathrm{NNP}(\mathrm{CD}(\mathrm{NN} \mid \mathrm{NNP}))(\mathrm{NN} \mid \mathrm{NNP}) \\
\text { Circa } 14 \mathrm{~km} \text { ENE }\end{array}$ \\
\hline S7 & $\begin{array}{l}\text { Directional part of } \\
\text { region }\end{array}$ & $\begin{array}{l}\mathrm{NP} \rightarrow \mathrm{JJ}(\mathrm{NNP} \mid \mathrm{NNS}) \\
\text { northeastern Roraima }\end{array}$ \\
\hline S8 & Topological relation & $\begin{array}{l}\mathrm{PP} \rightarrow \mathrm{IN} N \mathrm{NP} \\
\text { on Canal do Norte }[0030 \mathrm{~N} / 5030 \\
(\text { USBGN)] }\end{array}$ \\
\hline S9 & Distance & $\begin{array}{l}\mathrm{NP} \rightarrow \mathrm{CD} \mathrm{NN} \\
20 \mathrm{~km}\end{array}$ \\
\hline S10 & Approx. distance & $\begin{array}{l}\mathrm{NP} \rightarrow \mathrm{NNP} \text { CD NP } \\
\text { Circa } 25 \mathrm{~km}\end{array}$ \\
\hline S11 & Distance offset & $\begin{array}{l}\text { NP } \rightarrow \text { NP PP NP } \\
\text { Circa } 20 \mathrm{~km} \text { from Londrina } \\
{[2318 / 5109 \text { (USBGN)] }}\end{array}$ \\
\hline S12 & Direction offset & $\begin{array}{l}\mathrm{NP} \rightarrow \mathrm{NNP} \text { IN NP } \\
\mathrm{S} \text { of Franca }[2032 / 4724(\text { USBGN) }]\end{array}$ \\
\hline S13 & $\begin{array}{l}\text { Between two fea- } \\
\text { tures }\end{array}$ & $\begin{array}{l}\text { PP } \rightarrow \text { IN (NP CC NP) } \\
\text { between Rio Araguaia [0521/4841 } \\
\text { (USBGN)] and Braço Menor do Rio } \\
\text { Araguaia [0950/5012 (USBGN)] }\end{array}$ \\
\hline
\end{tabular}

pressions specific to tree structures (a.k.a. tree regular expressions or Tregex $^{5}$ ) for the development of patterns. It is important to note that this regular expression is different from regular expressions discussed earlier in Section 3.4 that were specific to regular expression utility of Java. Patterns developed using Tregex are used to find sub-trees of interest in

\footnotetext{
${ }^{5} \mathrm{http}: / /$ nlp.stanford.edu/software/tregex.shtml
} 
a collection of parsed descriptions. In this section we will now discuss tregex, tregex nodes, node relations and operators that can be used with relations.

As mentioned above, Tregex is a tree query language that uses regular expressions specific to tree structures for the development of patterns. The nodes are basic building blocks for the development of the tregex patterns. These nodes are available in the parse trees, e.g., NP, VP, NNP and others. Development of patterns follows a top-down approach by which we mean that first the non-terminal nodes must be identified and then the terminal nodes after which, the relationship they share can be identified. Non-terminal nodes can be expressed using node labels, i.e., NP, VP, and PP, which must match same labels in the parse tree. Similarly terminal nodes can be expressed by either using the node labels, such as NN, NNP, NNS and more or using regular expressions. The regular expression can be expressed between two forward slashes, i.e., /regexp/. For example, an expression /NN.?/ will match all terminal node labels which contain NN, such as NN, NNP or NNS. Similarly the information that a terminal node contains can be recognized by expressing standard regular expression over letters [A-Za-Z] and numbers [0-9]. In order to recognize other alphabets such as ã, Ã,ç, Ç and others they must be included in the regular expression. For example, to recognize a word Acará, the letter 'á' must be included in the standard regular expression which will then be [A-Za-z á]. These standard regular expressions can be used to constrain the type of values that matching node(s) can have. Another way of providing constraint is to use exact string, e.g., $\mathrm{m}, \mathrm{ft}, \mathrm{km}$ to match a very specific set of information.

Now that the nodes have been put in place in a pattern, it is important to specify relationships shared between these nodes in a parse tree. This allows patterns to be matched against the parse trees. Various relations can be expressed between nodes and it is important to note that all relations are relative to first node in a pattern. Here we explain some important relations (Table 3.4) that have been used in developing our patterns. The complete list of relations is given in Appendix B.

Table 3.4 Some base expressions for tregex node relations

\begin{tabular}{|c|l|l|}
\hline S.No & Symbol & Meaning \\
\hline \hline 1 & NP $<$ NNP & NP is a parent of NNP \\
\hline 2 & NP \$ PP & NP is a sister of PP (and is not equal to PP) \\
\hline 3 & CD \$- NN & $\begin{array}{l}\text { CD is immediate left sister of NN (both has } \\
\text { same parent node) }\end{array}$ \\
\hline 4 & NN \$+ NNP & $\begin{array}{l}\text { NNP is immediate right of NN (both has same } \\
\text { parent node) }\end{array}$ \\
\hline 5 & NP $<$ i PP & PP is the $\mathrm{i}^{\text {th }}$ child of NP \\
\hline 6 & NP $<-$ PP & PP is the last child of NP \\
\hline
\end{tabular}

In Table 3.4, the first node relation symbol is ' $<$ ' which specifies a 
parent-child relation between two nodes. For example, NP $<$ NNP means that node NP is a direct parent of node NNP and only those NP nodes will be matched who has NNP as its child and is highlighted as shown in Figure 3.7(a). The second relation symbol is ' $\$$ ' which means that a sister relationship can be specified between two nodes, e.g., CD \$ NNP signifies that CD is a sister of NNP (Figure 3.7(b)). The third and fourth node relations are used to specify more immediate sister relationships between nodes. For example, if we we want to match only those CD nodes which are immediate left sister of node NN then relation ' $\$-$ ' is used to form a pattern CD \$- NN as shown in Figure 3.7(b). Similarly relation ' $\$+$ ' can be used to specify immediate right sister of a node, e.g., NN \$+ NNP pattern will match those NN nodes whose immediate right sister is NNP as shown in Figure 3.7(c).

(a)

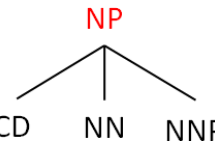

(b)

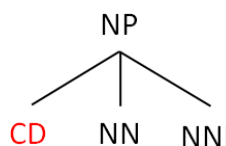

(c) $\mathrm{CD}$

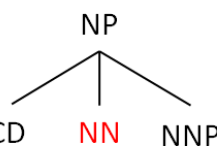

Figure 3.7 Red colored nodes in the tree above shows matched nodes using patterns for (a) NP < NNP; (b) CD \$ NNP and CD \$- NN; (c) NN \$+ NNP

Fifth node relation ' $<$ i' means that the pattern will match ' ${ }^{t h}$ ' child of the parent node. So, if NP $<4 \mathrm{PP}$, then the pattern would match that node NP whose fourth child is PP (from left to right). Sixth relation of '<-' means that the pattern would match that node NP whose last child is node PP.

When developing patterns, operators can be added to node relations to find more specific results. Operators such as conjunction ('\&'), disjunction ('|') and negation ('!') along with other tregex relations can be used in developing patterns. For example, node relations can be combined using '|' operator and pattern like NP $<\mathrm{NP} \mid<$ PP would match only those NP nodes who has either NP or PP as its children as shown in Figure 3.8(a). Similarly node relations can be negated using '?' operator e.g., pattern NP $<$ NP ! $<$ PP will match any NP node whose child is NP or any other node except node PP (Figure 3.8(b)). Node relations can be combined using the '\&' operator, e.g., NP > S \& \$++ PP, where pattern matches any node NP that is under node $S$ and has a PP as its right sister. Also node relations can be made optional by placing '?' operator, before them. For example, in NP $<$ NNP ? $<$ PP, node PP is optional in the pattern.

These operators can be grouped using square brackets '[' and ']'. For example, pattern NP $[<\mathrm{NNP} \mid<\mathrm{NN}]$ \$ PP will match any node NP which has its child either NNP or NN and with sister PP as shown in Figure 3.9.

The node(s) in the pattern can be named using '=' sign, e.g., NP=dis, meaning node NP is named as dis, which represents distance information when matching node is found in the parse tree. All named nodes are stored in a map that maps name(s) to node(s) and can be extracted when a corresponding match is found. The advantage of naming the nodes is that when building the complex patterns, these named nodes can be 
(a)
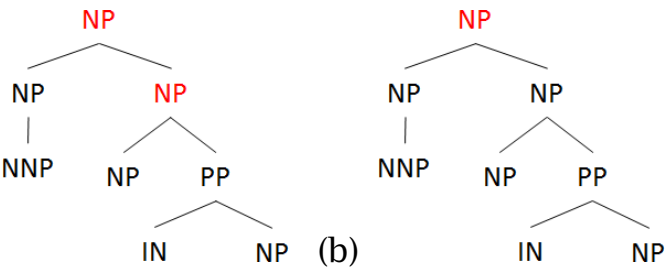

Figure 3.8 Red colored nodes in the tree above shows matched nodes using patterns for (a) NP $<$ NP $\mid<$ PP; (b) NP $<$ NP $!<$ PP

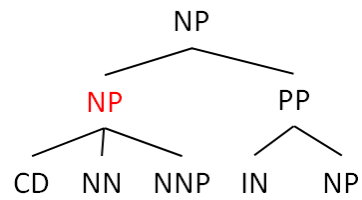

Figure 3.9 An example of matched node NP (in red) for pattern NP [ $<$ NP $\mid<$ NN] \$ PP

referred back to the pattern and multiple back-references are allowed. To refer to previously named node is possible using the link symbol ' $\sim$ '. Having come so far, let us now discuss some of the actual patterns that we developed for our work. We have called them Spatial Hint Linguistic Patterns (SHLP).

For representation purpose in this chapter the patterns have been modularised and the modularised expressions are shown in Table C.1. It makes it easier to read SHLP in Appendix C using modularised expressions described in Table C.1. Both the table and the SHLPs are given in Appendix C. It is important to note that each hint type may have one or more extraction patterns. For example, in Appendix C, pattern P9 Example: 1 and Example: 2 both aim to extract 'direction', 'place name' and 'geocode' from direction offset hint type but pattern in Example: 2 is designed to exclude extra words like 'city of' or 'town of' which is not required to be extracted.

Appendix $C$ gives an exhaustive list of all the patterns that we have used in this project. The patterns aim at extracting frequently occurring spatial hints from the descriptions. Only hints that represent qualitative information such as 'near', 'far', 'short distance from/to' has not been considered for creation of extraction patterns since these were not required for our purposes at this stage.

In Table C.1, the expression of CHAR is used in the patterns to match place names (e.g., Mato Grosso, São Paulo and others) in the parse trees. ORG matches organisation names (e.g., USBGN, ICWB), using words in all capital letters. The direction part of a region is matched using expression in PARTOF. The geocodes (e.g., 0940/6527 or 0014N/6829) of the place names are matched using expression of GEOCODE, and expression DIRREL is used to match words that represent a portion of a 
linear feature or some place (e.g., ' $N$ of lower Rio São Mateus' or 'NE of western Belém'). The VAL expression is used to match numbers that represent distance or elevation values. Similarly DUNI and EUNI matched the units of distance and elevation respectively. The compass directions are matched using expression in CDIR. The word 'circa' used before distance or elevation is matched using expression of APPROX and RELDIR expression matched relative directions, e.g., ' $25 \mathrm{~km}$ above Rio Negro' or 'below Manoá'. CAT is used to match words such as city or town that represent human settlements used in hint, like ' $24 \mathrm{~km} \mathrm{~N}$ of city of São Paulo' or 'W of town of Manacapuru'. The expression in PRE matches words 'central' or 'extreme', like 'central western Bahia' or 'extreme northern part of state' or 'northern central Bahia'. The EXT expression is used to match certain words that may be of interest but not required to be extracted at this stage.

Let us understand some patterns of Appendix C, pattern P1 is used to match a subtree of an elevation hint like those in Figure 3.10(a). The pattern can be interpreted as, any NP node with a child node CD, whose immediate right sister $(\$+)$ is a noun node with a constraint that it can have either ' $m$ ' or ' $\mathrm{ft}$ ' as its value and CD can have 'numbers' as its value for being a candidate pattern for elevation hint. The child node CD also uses negation relation, such that it does not have any other noun node as its left sister carrying 'circa' as its value. This differentiates the pattern in P1 with pattern in P2. Similarly, pattern P2 is used to identify subtrees with approximate elevation hints, as in Figure 3.10(b). Here, node NP has three children with first and the third node having noun tag and the second node tagged as CD. The constraint for the first node is that it can have only one value 'Circa', whereas the values for the second and third node are defined in the same way as in pattern P1.

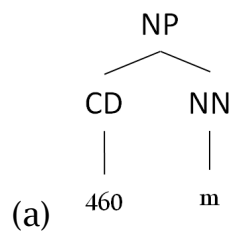

(b)

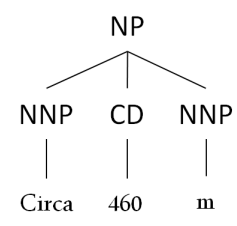

Figure 3.10 Subtree recognised by pattern: (a) P1 for elevation hint and, (b) P2 for approximate elevation hint

Pattern P3 is used for hints of type 'Placename geocode and organisation'. In P3, top node NP has five children of which the first and the third are NP nodes, the second is a left bracket node (-LRB-), the fourth is a parenthetical node (PRN), and the fifth is a right bracket node (-RRB-) (see Figure 3.11). All four nodes have child nodes, the first has singular noun tag with the constraint that its value is a text string using character set (CHAR) defined in Table C.1. The second and fifth phrase node have a left and right bracket tag, and the third node CD is constrained so that it uses expression of GEOCODE from Table C.1 to match geocode in the 


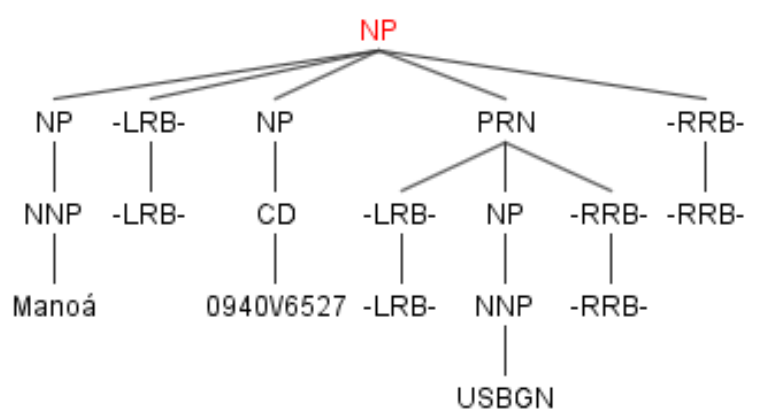

Figure 3.11 Subtree recognised by pattern P3 for hint 'placename geocode and organisation'. The ' $V$ ' in the tree above is actually ' $\backslash$ /'but looks like ' $V$ ' in print form

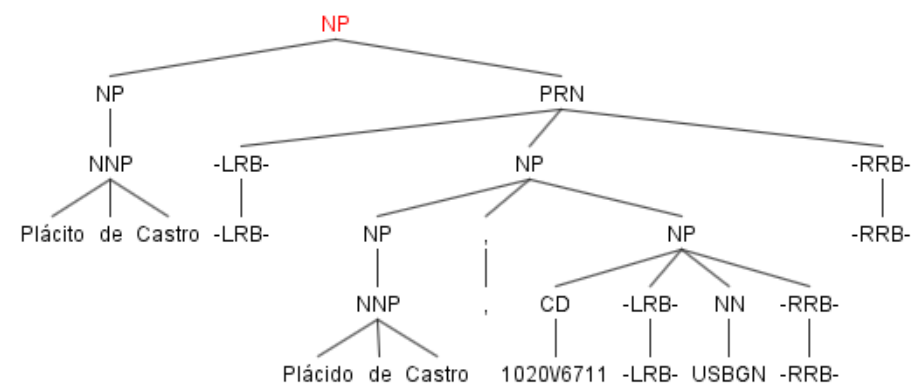

Figure 3.12 Subtree recognised by pattern P4 for hint 'placename geocode and organisation' with a mention of alternate spelling for place name

parse tree. The fourth node (PRN) further has three children of which we constraint the second child node so that it can only have a text string with capitalisation. In our corpus, some place names are mentioned along with their alternate names/spellings followed by their geocode and naming organisation. For example, Pláci to de Castro has variation in spelling as Plácido de Castro, as shown in Figure 3.12. We extract such place names using pattern $\mathrm{P} 4$.

Pattern P5 Example:1, is created by joining two subtrees. As shown in Figure 3.13, the first subtree (A) is for hint 'distance and direction' and the second subtree (B) for 'place name geocode and organisation'. The pattern used for subtree (A) is,

$$
\begin{aligned}
\mathrm{NP} & <((\mathrm{CD}<\mathrm{VAL}) ! \$-(/ \mathrm{NN} . ? /<\mathrm{APPROX})) \\
& <((/ \mathrm{NN} . ? /[\mathrm{CDIR}]) \$-(/ \mathrm{NN} . ? /[\mathrm{DUNI}]))
\end{aligned}
$$

which aims to find that node NP which has three children. The first child with node $\mathrm{CD}$, second child with node NN and the third child with any noun node represented by node expression /NN.?/. The first node 


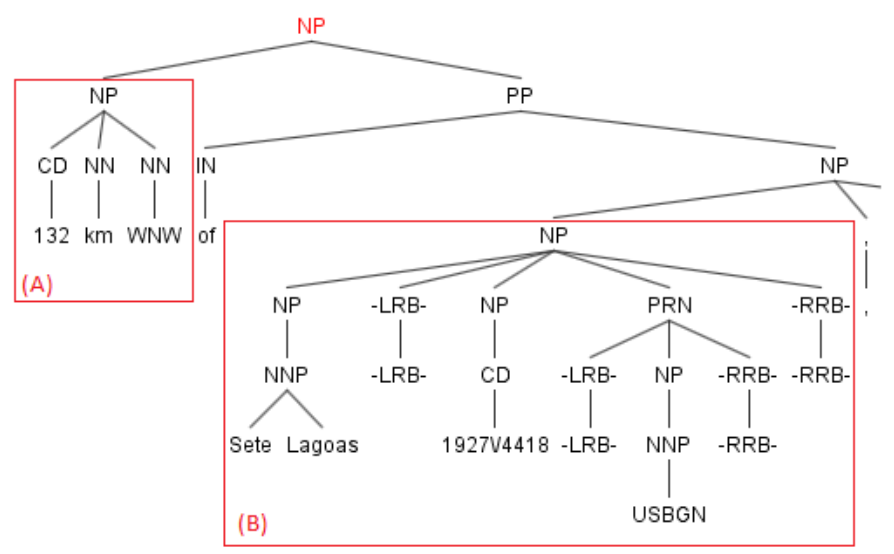

Figure 3.13 Subtree recognised by pattern P5 for hint 'distance direction from a reference point'

CD should not have any left sister with 'circa' as its value. But the third child must have a left sister that contains value of distance unit, i.e., ' $\mathrm{km}$ ' or ' $\mathrm{mi}$ '. This gives us a node NP for subtree (A) as shown in Figure 3.13. The second subtree pattern (B) is for placename geocode and organisation. To tie these two sub-patterns as a single pattern for hint type 'distance and direction from a reference point'. We use top node NP (in red color in Figure 3.13) which is parent to another node NP (of subtree (A)) and node PP. The pattern for these three nodes can be represented as

$$
N P<(N P \$+P P)
$$

where the child node NP (inside brackets) is a right sister of node PP. Here the left sister NP can be replaced by pattern we described for subtree (A) above. The node PP further has two children IN and NP and can be represented as,

$$
\begin{aligned}
\text { PP } & <1 \text { IN } \\
& <2 \mathrm{NP}
\end{aligned}
$$

Node IN can have value 'of' and node NP can further have two children, i.e., NP and comma (we have kept this second child as optional because in some trees there may be no second child). This NP node is a node that carries pattern for subtree (B). Putting pattern PP (3.4) inside NP (3.3) above, the pattern will now become,

$$
\begin{aligned}
N P<(N P \$+(P P & <1(I N<\text { of }) \\
& <2(N P<1 \mathrm{NP})))
\end{aligned}
$$


The bold NP node is a parent node for placename geocode and organisation. There is one exception to this pattern when compared to the pattern in P3, which is, that we constrain the first node carrying the placename using negation so that it does not have any adjective nodes as its left sister. The reason for this is to avoid overlap with other patterns. The placename geocode and organisation pattern now becomes

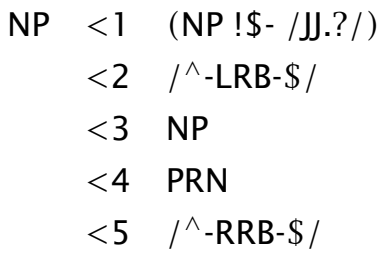

If we put pattern (3.2) and (3.6) in pattern (3.5) then we get the final pattern P5 for Example:1 as shown in Appendix C. Likewise all other patterns in Appendix C can be understood and interpreted.

As we move down the list of patterns they become more complex. There is more than one way of developing patterns to achieve desired results and it varies with personal expertise and choice. We took utmost care in developing patterns such that maximum variation in the descriptions can be covered and that patterns do not clash with each other. These patterns are input to the extraction engine for the identification of spatial hints in parse trees, and this is described in the following section.

\subsection{Information Extraction pipeline}

Information Extraction (IE) is a process of extracting domain-specific information from natural language text, and is considered to be a subfield of NLP (Jackson and Schilder, 2006). There exist many domains where IE can be applied, such as medical, current news, spatial information, crime and many others. In this project, we use IE for the spatial domain and call it Spatial Information Extraction (SIE). A concise definition of what IE covers is given by Moens (2006), based on the definitions by Riloff and Lorenzen (1999) and Cowie and Lehnert (1996) "Information extraction is the identification, and consequent or concurrent classification and structuring into semantic classes, of specific information found in unstructured data sources, such as natural language text, making the information more suitable for information processing tasks."

Usually, in any information extraction task, it is known in advance which kind of information is to be extracted and this information is stored in templates. The template consists of a collection of attributes, which may be filled by one or more values from the extracted information. A template is a table with attributes in a database. Generally, the extracted information adheres to some predefined category, e.g., person, places, organisation but this may vary with the domain of application (Moens, 
2006). For example, when extracting information for terrorist activities, one might be interested in type of incident, perpetrator, physical target, human target, instrument and location of incident (Jackson and Schilder, 2006). Similarly in our case, for example, with the hint type of distance direction from a reference point, we are interested in extracting distance from the reference point, direction from the reference point, name of the reference point and the geocode of the reference feature. This extracted information allows computations to be done on previously semi-structured texts. The typical components of an information extraction system are shown in Figure 3.2, which extends the system of NLP by adding two modules: extraction engine and template filling.

While NLP is more of a domain-independent task in which linguistic entities such as words, sentence boundaries, POS tags and syntactic analyses are recognized, the task of information extraction goes one step further, by identifying domain-dependent information using patterns. These patterns are developed based on a priori knowledge from domain experts and are used to extract important information from text documents (Moens, 2006).

\subsubsection{Extraction engine and Template filling}

In this section we discuss step-4 and 5 as indicated in Figure 3.2. The main task of the extraction engine is to identify and extract information of interest from gazetteer entries for their included various hint types. For this, it takes two inputs - the first is the parse tree and the second is a set of patterns which are used to identify nodes that carry information in the trees. The output from an extraction engine is a set of information units that can be stored in simple data structures by attribute and their value.

Before we move forward it is important to recall that given a gazetteer entry, its description may contain one or more hint types and each hint type contains number of information units that need to be extracted. To extract those information units we develop patterns per hint type.

The contained information units per hint vary. For example, in the direction offset hint, 'SE of Serra de Baturité [0415/3905 (USBCN)]' we want to extract four pieces of information, i.e., its direction 'SE', the placename with respect to which the direction is referred 'Serra de Baturité', its geocode '0415/3905' and organisation 'USBGN'. We call these information units. Sometimes, this hint uses a word for the part (upper, lower, middle) of a feature. An example is 'NE of upper Rio Ribeira de Iguape [2440/4724 (USBGN)]', and 'upper' also must be extracted, as it provides important information about the locality. Similarly, in the distance and direction from a reference point hint ('30 km NNW of Juquiá [2419/4738 (USBGN)]') we want to extract information about distance ('30 km'), direction ('NNW'), placename ('Juquiá'), geocode ('2419/4738') and organisation ('USBGN').

The extraction engine identifies information units in the parse tree, and stores their values in the dedicated hints table. Each hint type has a 


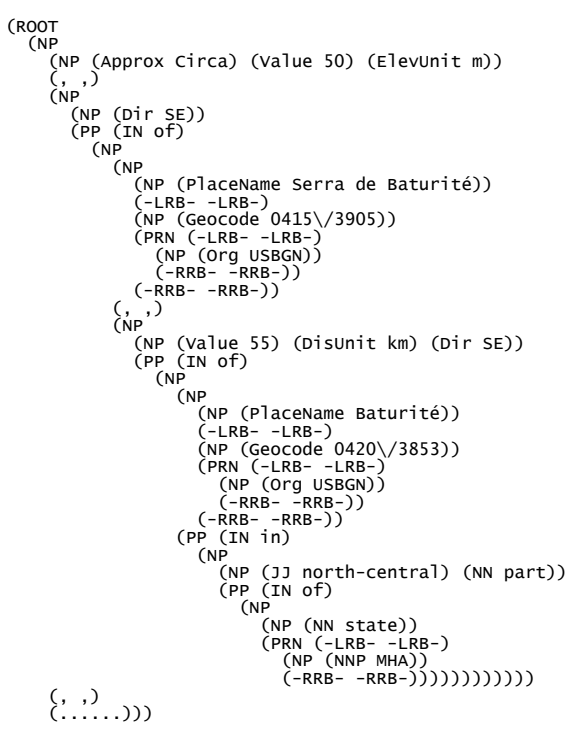

Figure 3.14 Excerpts from a tree with relabeled nodes

separate table in which the information units are stored by the extraction engine.

Information unit identification involves tree-pattern matching and node-manipulation. For each hint type, there is a specific dictionary, in which its patterns are stored. The dictionary is flexible, thus patterns can be updated, appended or deleted. The nodes carrying the information unit in the pattern are named (as discussed in Section 3.6.1), to identify which of them (carrying information units) are matched.

The developed patterns are expressed in the Tregex, and pattern matching is done with the Tregex engine. Subsequently, identification (labeling) happens with the Tsurgeon engine, which manipulates the parse trees. Once a matched node(s) is found in the parse tree, it is subsequently handled by an appropriate Tsurgeon operation, and it becomes a transformation node for Tsurgeon operations (Levy and Andrew, 2006). The two engines form a powerful tree manipulation language tool, which can operate on arbitrary tree structures.

For manipulation by Tsurgeon, certain operations (delete, relabel, insert, coindex, move, excise, prune, insert, replace and adjoin) are available for tree structure modification (Levy and Andrew, 2006). Since our interest is to extract information units from matched patterns, we only apply the relabel operation. This operation changes the label of the matched named nodes in the parse tree without affecting its structure, as shown in Figure 3.14. Matched nodes are relabeled for easy identification in the next phase, indicating their correspondence to attributes in the spatial hints table.

The parse tree is traversed once again, and all identified information units are extracted. Each information unit is a simple (attribute, value) 
pair, e.g., placename = Juquiá. Multiple information units form a spatial hint, and each spatial hint is inserted as one row in the spatial hints table. A parse tree of a single gazetteer description usually contains multiple spatial hints, thus triggering the insertion of multiple rows in the spatial hints table. For example the spatial hint type 'direction offset spatial hint' has the following attributes: compass_direction, part_of, place_name, geocode, organisation, and the information units will provide values for most, if not all, of these. Forming spatial hints from information units is done by traversing the parse tree in the order of the sentence as follows:

- Consecutive information units of the same spatial hint type are assigned to a single spatial hint of that type (e.g., distance = '12', unit $=$ ' $\mathrm{km}$ ', direction $=$ 'SE', placename $=$ 'Curitiba', geocode $=$ '2525/4915' will form a single spatial hint)

- When same pattern is matched more than once in a parse tree, this marks a new record in hint table and new informations units are inserted in it.

GlobalVariable: a set of information units that are gathered and consumed while the algorithm runs over the parse tree.

Input: a node of the modified parse tree

Procedure: the set of information units extracted from this node and all child nodes are gathered. However, the moment that an information unit is encountered that is already in the set, the information units gathered up to this point are considered to be a complete spatial hint, which is immediately stored as a record in the table. Then, the set of information units is re-set to an empty set, before adding the new information unit. Post condition: the set of information units that are gathered since the most recent 'store' are delivered. They form a spatial hint that is not yet stored in the hints table.

Function: readSpatialHints(node)

BEGIN

if (node.name is an information unit) then

if (node.name already in current spatial hint) then

write out spatial hint;

create a new empty spatial hint;

insert(node.label, node.value) in it;

else if (node.name in set of attributes that mark a new spatial hint)

then

write out spatial hint;

create a new empty spatial hint;

insert (node.name, node.value) in it;

else

I insert (node.name, node.value) in current spatial hint end

end

for each child node in node.children do

I readSpatialHints(node)

end

END

Algorithm 1: Hint extraction algorithm 
This extraction process can be translated in the following pseudocode (Algorithm 1), which describes a recursive function that traverses the tree and gathers all information units found into spatial hints. The function is to be called giving the root node of the relabeled parse tree, whereby the nodes that form information units are labeled appropriately.

Each hint type has a separate table in which information units are stored. For example, Table 3.5 and 3.6 shows the hint tables for 'direction offset' and for 'distance and direction from a reference point' hint type, respectively.

In Table 3.5 and 3.6, the count attribute represents the number of different matches of a specific extraction pattern in the same parse tree for a specific hint type, i.e., if the count is 2 for some gaz_id, then a pattern is matched twice in a parse tree. Attribute gaz_id identifies the gazetteer entry, dir indicates to cardinal direction, placename indicates the feature name, with reference to which dir or dist is used, geocode of the placename and org refers to the naming standardisation organisation used in this entry. In Table 3.6, attribute approx is used whenever the distance value is approximate.

Table 3.5 Direction offset hint table in our database.

\begin{tabular}{|c|l|l|l|l|l|l|}
\hline count & gaz_id & dir & partof & placename & geocode & org \\
\hline \hline 1 & 24 & SE & & $\begin{array}{l}\text { Serra de } \\
\text { Baturité }\end{array}$ & $0415 / 3905$ & USBGN \\
\hline 1 & 1117 & NE & upper & $\begin{array}{l}\text { Rio Ribeira } \\
\text { de Iguape }\end{array}$ & $2440 / 4724$ & USBGN \\
\hline
\end{tabular}

Table 3.6 Distance direction from a reference point hint table in our database.

\begin{tabular}{|c|l|l|l|l|l|l|l|}
\hline count & gaz_id & approx & dist & dir & placename & geocode & org \\
\hline \hline 1 & 24 & & $\begin{array}{l}55 \\
\mathrm{~km}\end{array}$ & SE & Baturité & $0420 / 3853$ & USBGN \\
\hline 1 & 933 & circa & $\begin{array}{l}30 \\
\mathrm{~km}\end{array}$ & NNW & Juquiá & $2419 / 4738$ & USBGN \\
\hline
\end{tabular}

The extraction of information units from gazetteer descriptions allows to subsequently deploy spatial computation machinery useful in other application domains. In this project, these information units are used as input parameters for interpretation of hints by developing spatial representations and functions that calculate uncertainty associated with each hint. Their utilization is discussed in Chapter 4 and 5.

\subsection{Evaluation and Results}

Once patterns for information extraction have been prepared, it remains to evaluate the performance of our patterns. Traditionally, the measures of recall and precision have been used to assess the performance of 
the information extraction systems (Grishman and Sundheim, 1996; Moens, 2006). Recall is the ratio of number of relevant entries retrieved for a specific hint type during the extraction process over the number of relevant entries containing that specific hint type in the database, whereas precision is defined as a ratio of relevant entries retrieved for a specific hint type over all the entries retrieved during the extraction process. For our extraction results it is important for our patterns to not just extract information with high recall but is also important that that information contains no or very few errors. To measure the recall and precision, following formula (van Rijsbergen, 1979) can be used:

$$
\text { Recall }=\frac{\text { relevant } \& \text { retrieved }}{\text { relevant }}, \quad \text { Precision }=\frac{\text { relevant } \& \text { retrieved }}{\text { retrieved }}
$$

We first calculated the recall scores for our patterns. For each hint type, we first ran a query over all the gazetteer entries in our database to arrive at an exhaustive list of descriptions that contain a specific hint type. This gives us the total number of 'relevant' entries. We also had the tables containing the extracted information in which it is possible to see which entries were retrieved. Then we matched the unique ids of retrieved entries with the relevant entries to look for those entries that may or may not be relevant. These entries were then painstakingly checked and appropriately kept or removed from the retrieved table. We then counted the total number of relevant and retrieved entries and further calculated the recall scores of patterns for each hint type. The results are shown in Table 3.7.

Table 3.7 Pattern evaluation results, in which $r$ \& $r$ represents relevant and retrieved entries

\begin{tabular}{|l|c|c|c|}
\hline Hint type & r \& r & relevant & recall \\
\hline \hline Elevation & 1454 & 1874 & 0.7758 \\
\hline Between two features & 96 & 201 & 0.4776 \\
\hline Direction offset & 374 & 492 & 0.7601 \\
\hline $\begin{array}{l}\text { Distance direction along/from a reference } \\
\text { line }\end{array}$ & 67 & 126 & 0.5317 \\
\hline Distance direction from a reference point & 1523 & 1739 & 0.8757 \\
\hline Direction part of region & 2053 & 2300 & 0.8926 \\
\hline
\end{tabular}

As mentioned previously in Section 3.6.1, to capture the variation in some hints, more than one pattern has been used to extract information units. However, in the results table above we only report the aggregate results for hint types respectively.

Since there is no threshold value of recall score, beyond which the scores may be considered good, we consider a recall score of 0.75 as a threshold and observe that most of the patterns for hint types have a good recall score. For cases with lower scores, we investigated and found that some factors could be responsible for a low recall scores including 
reasons like parser failing to generate the parse tree when the length of the description is too long and that some times wrong tags are assigned at word or sentence level, e.g., NN (noun tag) instead of JJ (adjective tag).

We then tried to evaluate the precision of our extracted results. However, for evaluating the precision an exhaustive list or compilation of the correct hint tables is required which becomes the base table against which the extracted results can be compared. For our corpus, we did not have such base tables and hence it was not possible to evaluate the precision in the results. During our extraction process however, we have ensured to make it as accurate as possible by putting sufficient and relevant constraints in the patterns such that accurate information units get extracted from the parse trees and stored in specific columns in hint tables as discussed in previous section. To further check our results we ran various queries over those tables to make sure that in those hint tables, each column has only the expected information. For example, we checked using queries that the distance column in the table contained distance information only. With these checks in place we expect negligible occurrence of error.

\subsection{Summary}

In last chapter we identified various types of spatial hints available in our gazetteer descriptions. To do any spatial computation or analyses with them we first needed to extract them and store them in a structured format, which was the main aim of this chapter. In this chapter, we explained our approach and implementation of extracting information from hints using techniques from Natural Language Processing (NLP) and Information Extraction (IE). We were aware that our descriptions have compound names, abbreviations and extra punctuations, which is why, they needed to be normalised in order to get correct parse trees.

After that we passed the descriptions to the NLP pipeline where various components of NLP worked on the input descriptions to produce the syntactic structure in the form of a parse tree. These parse trees were then analysed to identify hint specific subtrees that carry information. In order to extract this information we needed some patterns that can be matched against the parse tree. For this purpose we used tree query language (Tregex) to develop hint specific patterns.

The parse trees and the developed patterns were used by the extraction engine whose main task was to find matches in the parse tree against the hint pattern under consideration. Once the match was found, our simple hint extraction algorithm extracted the information and stored them in a predefined data structure in the database.

To evaluate the performance of our developed patterns, we used recall scores and found that patterns for most of the hint types have a good recall score. Finally, the extracted information is used in Chapter 4 to develop spatial extents for a specific hint type. 



\section{Spatial extent models for directional containment phrases}

\subsection{Introduction}

In Chapter 3, we extracted various spatial hint types of which 'directional containment' can be found in 2,300 entries. This hint type cover phrases like 'in southeastern part of state' or 'in southern central Minas Gerais' or 'extreme southern Rio Grande do Sul' as shown in Figure 4.1.

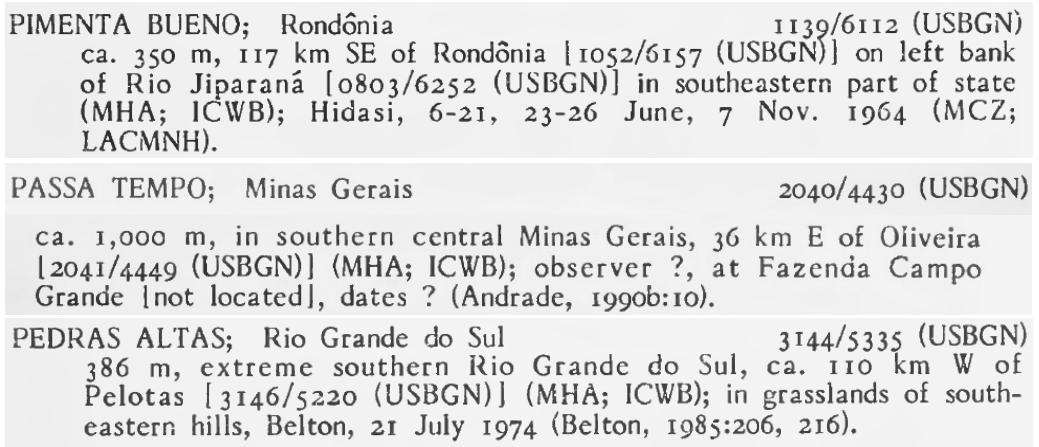

Figure 4.1 Example entries from the gazetteer (Paynter and Traylor, 1991b) showing directional containment hints

Since these phrases form more than half of the total descriptions we decided to construct spatial extents for this hint type so as to be able to locate as many localities as possible within those extents. To do so we need to further understand our descriptions and build our understanding on related work.

Within these directional containment hints, 25 directions were found. These directions can be categorized as cardinal direction, ordinal direction, half-direction, and extreme direction, and are further described in Section 4.3.2. The first two categories are standard compass directions, whereas half-directions and extreme directions are non-standard direction notions. We used entries containing directional containment hints 
extracted in previous chapter. In this chapter, we focus on constructing spatial extents for directional containment cases as mentioned above.

In Section 4.2, we discuss the work done by others using directional relationships, and Section 4.3 describes the rationale for our spatial extent models. The construction of spatial extent models is presented in Section 4.4. In Section 4.5, we look at the performance of these models, while Section 4.6 discusses our findings, followed by the summary of this chapter.

\subsection{Related work}

Location descriptions have been with us for a long time, and a large amount of such information has historically been collected. An important case is that of specimen labels in museum collections of biological specimens, and that of travel logs of expeditioners of the past. Such descriptions exhibit a variety of spatial relations between features (Wieczorek et al., 2004). In natural language, identification and understanding of spatial relations is important, and is fundamental to building geospatial semantics (Arpinar et al., 2006). The interpretation of spatial relations from natural language phrases is complex because of variation in human perception, and mapping to formal models is hard (Mark and Egenhofer, 1994; Schwering, 2007). Three types of spatial relations have been studied extensively: topological relations, direction relations and distance relations. In this work, we focus on direction relations.

The position of an object can be expressed using direction relations. Such a relation specifies how one object is placed with reference to another. Levinson (2003) distinguished two reference systems through which, direction relations can be expressed, namely a relative and absolute reference system. In the first, direction is expressed using the orientation of a reference object using words like right-of, left-of, frontof or back-of, whereas in the second system use is made of compass directions like east, west, northeast and others. Various approaches have been proposed for the interpretation of directions in both relative and absolute reference systems. For instance, the relative systems of double-cross calculus (Freksa, 1992; Zimmermann and Freksa, 1996) and rough graph approach (Werner et al., 2000) are extensively used in conducting orientation studies in robotic navigation tasks. Two absolute reference systems, are the cone-based (Haar, 1976) and projection-based systems (Frank, 1996; Papadias and Egenhofer, 1997; Theodoridis et al., 1996). In the first, a reference object is approximated as a point (centroid of an area) and angular lines originating from it help determine in which direction cone the target object falls. The cone-based approach is robust for objects but is imprecise when the target is not a point. This was later improved in the Minimum Bounding Rectangle (MBR) approach of (Peuquet and Zhang, 1987). That model proposed that instead of a point, its MBR should be used to generate the direction tiles. Subsequently, (Goyal and Egenhofer, 2000) proposed an envelope approach to 
represent cardinal direction relations between disjoint non-point objects. In that approach, partition lines of the envelope of the reference object are extended until they intersect the target object, or the target object falls in a direction sector of the reference object. This approach clearly gives unequal sectors that depend on shape of the reference object (see Figure 1 in (Goyal and Egenhofer, 2000)). Some other challenges of that model were discussed by (Skiadopoulos and Koubarakis, 2004), whose approach did not approximate a region to a point. Both approaches were later assessed by Frank (1992) who concluded that the MBR-based approach is superior.

Most research in direction relations between objects aim to provide formal methods. In contrast, Dehak et al. (2001) first proposed to evaluate the probability of a point of being in a specific direction from another point. Their approach provides the algebraic formulation of probabilistic point distribution through which positional probability can be calculated. Later, the probability approach was also used when positional information is incomplete or inaccurate (Dehak et al., 2005). Based on the above approach, Guo et al. (2008) suggested to include an uncertainty field to represent the target object, especially when positional information is imprecise. Likewise, Sun (2008), investigated the deficiencies of Goyal and Egenhofer's method of modeling cardinal direction relations between simple regions, and proposes to use fuzzy set theory in conjunction with mathematical morphology to refine the cardinal direction relations, which considers the shape of the reference region as well as its fuzziness. Bloch (2006); Lucas (2012), used knowledge representation and formal modeling structures to convert verbal spatial information into geographic representations. They used knowledge representation to understand domain-specific information, whereas formal modelling using fuzzy membership functions was used when the information presented is vague, e.g., ' $250 \mathrm{~m}$ east of $X$ '. Vagueness has been extensively researched in the geospatial domain and is used where basic crisp models cannot be applied. Some of the early models were proposed by Cohn and Gotts (1996), Clementini and Felice (1997) and Schneider (1996).

So far, all the approaches discussed above identify directional relationships between two objects. However, very few studies have addressed finding some part of a known region when that part is described using directional relations, e.g, ' $X$ in eastern part of 〈region〉' or ' $X$ in southeastern part of 〈region〉.' Though Region Connection Calculus (RCC) (Cohn et al., 1997) and 9-intersection (Egenhofer et al., 1994) models provided formal containment relations between two objects, they do not apply in expressing directional containment as defined here. Though that work has been very influential, we do not believe it to be so closely related to our work. We are not studying the relationship of two arbitrary regions, but rather the possible spatial interpretation of text phrases that describe a single region by mentioning another known region.

In contrast, our work is more related to the work by Liu et al. (2005) that addressed the problem of identifying position of an object within 
a region by first determining its directional sectors. They offered three approaches, one of which is similar to the model of (Goyal and Egenhofer, 2000). Aiming to delineate the central sector, in a cognitive experiment, subjects were asked to assign a direction and level of accuracy to a number of points presented on a map (Liu et al., 2005). They used a value $\rho$ as scaling factor by which the bounding box of a region would need to be down-scaled to obtain a central sector box for that region. Varying over values between $\frac{1}{2}$ and $\frac{2}{3}$, a value of $\rho=\frac{1}{3}$ was determined as best fitting with the experiment subjects' cognition.

The problem of determining direction-based sectors was also addressed in van Kreveld and Reinbacher (2004), who addressed only the four cardinal directions. They presented criteria for splitting the original region, and efficient algorithms for determining the extents of sectors, meeting those criteria. Our work is less theoretically algorithmic, but is an attempt to address more pragmatically the same problems for a more complete range of directions, while evaluating different models against a body of data, thus providing a complimentary view of the problem.

\subsection{Background for constructing spatial extent models}

\subsubsection{Terms explained}

It is imperative to understand some important terms used in this chapter before we go ahead. The term region identifies any area of arbitrary shape, having a known extent, and the area size of which can be calculated, such as Paraná, a state in Brazil. A sector (a.k.a. compass sector) is a part of a region and we have used two sector types namely: central sector and outer sector. The 'central sector' is the central part of the region and all other parts of the region are called 'outer sectors'. For example, 'northeastern Paraná' refers to the 'northeastern' part in the region of 'Paraná' and we call it 'outer sector' in this chapter. Similarly, 'central Paraná' is the 'central' part in the region and is referred as 'central sector'. The set of all sectors in the region constitutes a model for that region. The determination of such a set is the model construction for that region; model construction is explained in Section 4.4.

\subsubsection{Direction types}

We identified three types of direction phrases in our gazetteer corpus. The definitions and examples of each type are provided below.

Full directions are directions are phrases that make use of cardinal or ordinal directions. The cardinal directions form the main four directions of the compass rose (n, e, w, s), whereas the ordinal directions represent the other four directions of the compass rose (ne, nw, se, sw). We add 'central' (abbreviated as 'c') as a special 
case of cardinal directions, as many entries in our corpus refer to it.

Half-directions are those direction phrases that are a conjunction of cardinal directions and the phrase 'central'. There are two types of half-direction: central half-directions ('northern central) and noncentral half-directions ('central northern') Singh and de By (2012). We abbreviate these two types as ' $\star c$ ' for central half-directions and ' $\mathrm{C} \star$ ' for non-central half-directions.

Extreme directions are those direction phrases that use the phrase 'extreme' as a prefix to cardinal or ordinal direction, e.g., 'extreme northern Bahia', 'extreme southwestern Bahia'. We assume that the extreme direction represents that part of the cardinal (or ordinal) direction that is closer to the outer border of the sector.

Of all the entries containing directional containment phrases, $65 \%$ are full directions, $26 \%$ are half-directions and extreme directions are $8 \%$.

\subsubsection{Organisational principles for spatial extent models}

We have shown the different types of directions in our corpus and how we see them in a hierarchical tree in Figure 4.2. The first direction type is of cardinal directions. The second type addresses the type of ordinal directions. These are the directions that we use to interpret phrases such as 'southeastern Paraná.' As a subtype of the cardinal directions, we identify the half-directions because they are the conjunction of a cardinal direction and the phrase 'central', as in, 'central northern' or 'northern central.' Another subtype is called extreme directions, and these are derived from both cardinal and ordinal directions, as in, phrases such as 'extreme northern Paraná' and 'extreme northeastern Paraná' respectively. We want to spatially interpret these direction types and for this purpose, we create spatial extent models that are a conjunction of sectors in the region. To fully create the model in a region, it must have a choice of placement, specific shape, and size, the respective details of which are covered in Section 4.3.4.

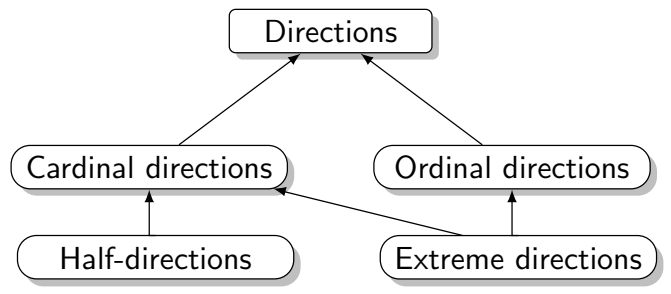

Figure 4.2 Directional containment phrase hierarchy used in this work

We identified principles that are valid for model construction in all three direction types. These are:

- every sector falls within its region, 
- the definition of a model is fully determined by the choice of a number of parameters, which are subsequently identically applied to all sectors of the same type, i.e., the determination of sector extents is deterministic,

- spatial extent models consist of choice of centre, central sector, and outer sectors,

- the determination of extents of outer sectors is done symmetrically, by using constant grid sizes or constant angles,

- the choice of centre (position) serves as an anchor for later choices of sector in the same model, and

- angle computations take place in a geographic reference system, while length and area computations take place in a (metric) projected reference system.

\subsubsection{Model characteristics}

Our model construction is built around three important characteristics. They are placement, shape, and size.

It is important for models to have a placement in any arbitrary region. We studied four different placement options. Each determines where to position the region's centre point. A choice here, functions as an anchor for placement of sectors.

region mass centre $(\mathbf{r m c})$ is the centre of mass of the region,

circle centre (cc) is the centre of the minimum bounding circle around the region,

envelope mass centre (emc) is the centre of mass of the region's envelope,

mass box centre (mbc) is the centre of the mass box which is obtained by determining the horizontal and vertical $\frac{1}{3}$ and $\frac{2}{3}$ mass cut lines of the region, which together give rise to a roughly one-ninth-of-region central sector, of which we determine the centre of mass.

Every model also needs to make two fundamental choices considering shape. These choices affect the fundamental shape of, the central sector as well as the outer sectors. The central sector can have one of a list shapes: hull, circle, and others discussed in Section 4.4.1.1 below. The outer sectors are shaped either using angular lines that originate from the choice of centre in the region, or using some kind of regular grid.

The size defines the spatial extent of the sectors in the region which have a bearing on the model performance. Construction of models for different direction types and different shapes leads to different sectoral sizes. The optimality of these sizes were chosen after detailed analyses explained in Section 4.4. 


\subsection{Construction of spatial extent models}

The actual models are constructed under the principles for model construction, as discussed in Section 4.3. In the following sections, we describe how we construct these models for different direction types. The general approach is that we choose a location for center, then we decide on the shape and size of the central sector and place it at the chosen center, and that we subsequently drape the outer sector around central. Shape and size of both half-direction and extreme direction sectors, though with variation as we shall see, follow naturally from those earlier choices.

\subsubsection{Models for full directions phrases}

\subsubsection{Models of central sector}

As discussed in Section 4.3.2, we include 'central' as a specific case of full direction since many entries in our corpus refer to such sectors. This is important to interpret phrases such as "central Mato Grosso."

The three characteristics discussed in Section 4.3.4 are used to construct the central sector. For the shape of the central sector, we use circle, clone, hull, oval and boxes, and for placing the central sector we can use the different placement options of Section 4.3.4. Since full directions are nine mutually exclusive cases, with 'central' as one of them, we initially create the size of the central sector in such a way that it occupies $\frac{1}{9}$ of the total mass of the region. Then we use backintersection, which is a process that preserves the common area between the scaled down sector and the region. With a number of options on shape as well as placement, it is possible to create different models from their combinations. We discuss below various shape models for central sectors, and these are illustrated in Figure 4.3:

Circle the central sector is determined by a circle ${ }^{1}$ enclosing the region. To obtain the circle central sector, the circle model is scaled down to exactly one ninth of its region size. This circle central sector is back-intersected with the region, giving the circle central model.

Clone the sector uses a clone of the region, and, like the circle sector, is scaled down to one ninth of the region size. The clone central model is obtained from back-intersection of the clone sector with the region.

Hull the sector uses the convex hull of the region, scaled down to one ninth of the size of the region. The hull central model is obtained by back-intersecting the hull sector with the region.

Oval the central sector constructs an ellipse at region mass centre that is aligned with the dominant direction in the region. The longest diagonal distance chord $(l d d c)$ as well as its perpendicular distance

\footnotetext{
${ }^{1}$ In essence by 'circle' we mean a disc, namely a 2-dimensional feature rather than a linestring. However for reasons of simplicity we use the word 'circle' in this chapter.
} 
chord $(a p d c)$ are both determined with region mass centre as origin. The ratios of their length determines the ratio of semi-major and semi-minor axes of an ellipse: $\frac{|l d d c|}{|a p d c|}=\frac{a}{b}$. This is used to obtain the oval central sector, which is one ninth the size of the region. By back-intersecting this sector we obtain the oval central model.

We have two more models that do not require scaling and have a fixed placement in the region. They are: envelope-based and mass-based models.

Envelope sector makes use of a rectangle that minimally encloses the region in it. The envelope is equally cut by lines at one third and two third of its height and width, which results in a $3 \times 3$ grid of equally sized rectangles. The central sector of the grid is back-intersected with the region, giving the envelope central model.

Mass sector is created by cutting latitudinally and longitudinally at one third and two thirds of the region's mass. Similar to the envelope model, the mass model also results in nine rectangles, of which the middle one is back-intersected with the original region, to obtain the mass central model.

(a)

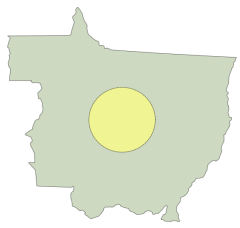

(d)

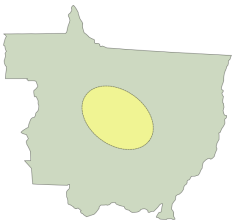

(b)

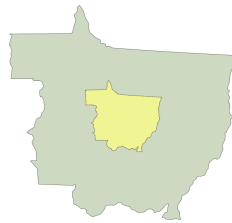

(e)

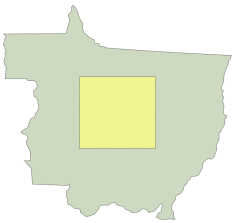

(c)

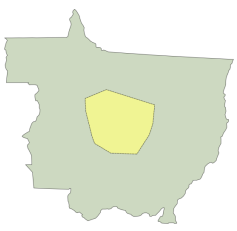

(f)

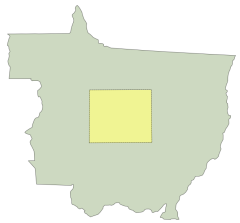

Figure 4.3 Different central sectors for Mato Grosso state, placed all at region mass centre. (a) circle; (b) clone; (c) hull; (d) oval; (e) envelope-box; (f) massbox.

Table 4.1 Placement options for centre/central sector combinations

\begin{tabular}{|lr|c|c|c|c|c|c|}
\hline center & $\begin{array}{r}\text { central } \\
\text { acronym }\end{array}$ & $\begin{array}{c}\text { circle } \\
\text { circ }\end{array}$ & $\begin{array}{c}\text { clone } \\
\text { clon }\end{array}$ & $\begin{array}{c}\text { hull } \\
\text { hull }\end{array}$ & $\begin{array}{c}\text { oval } \\
\text { oval }\end{array}$ & $\begin{array}{c}\text { envelope } \\
\text { ebox }\end{array}$ & $\begin{array}{c}\text { mass } \\
\text { mbox }\end{array}$ \\
\hline rmc & $\begin{array}{r}\text { mass } \\
\text { cc }\end{array}$ & $\square$ & $\square$ & $\square$ & $\square$ & & $\square$ \\
emc & $\begin{array}{r}\text { circle } \\
\text { envbox }\end{array}$ & $\square$ & $\square$ & $\square$ & $\nabla$ & & \\
mbc & massbox & $\square$ & $\square$ & $\square$ & $\square$ & $\square$ & \\
\hline
\end{tabular}

As per the constraints imposed in Section 4.3.3, we want the central sectors to fall inside the region. However, we identified that not all combinations of shape/placement/size give plausible combination especially 
with centre and central sector. We want all sectors i.e., central and outer sectors to fall inside the region, but with imposed constraints, not all combination of shape/placement/size accord with this condition. We have seen that certain combination extends the central sector beyond the region's extent, and for the same reason we had to drop them from our list. Table 4.1 shows those plausible combinations of shape/placement that were used for the construction of central models. Our algorithms ensure that after intersecting with the region the central sector is positioned and scaled in such a way that the size of the central sector is one ninth of the region.

\subsubsection{Models of outer sectors}

The models of outer sectors are used in interpreting directions, such as "eastern" or "southeastern." Similar to models of central sectors, here also, we apply the three model characteristics, shape, placement, and size.

For shape of the outer sectors, we use grid-based and angle-based outer sectors. The grid-based outer sectors are constructed using different methods, i.e., envelope mass distribution for the envelope-box model and region mass distribution for mass-box model. Both models combine naturally with the envelope central and mass central models. Placement of outer sectors makes a choice of one of the four centres identified in Section 4.3.4. The size characteristic is not chosen, but is fully determined by choice of outer sector model, and choice of centre. Outer sectors result from intersections of outer sector models with original region. We propose three outer sector models below.

Envelope grid outer sectors use the same technique as used in envelope central model above i.e., using cut lines at $\frac{1}{3}$ and $\frac{2}{3}$ of the envelope's height and width. Again, this gives us nine sectors of which the central one is the envelope central sector and the remaining eight are the envelope grid outer sectors (Figure 4.4(a)).

Mass grid outer sectors use the same technique as used in mass central model above, i.e., with cut lines at $\frac{1}{3}$ and $\frac{2}{3}$ of longitude and latitude of the region's mass distribution. Here too, we get nine sectors, of which the outer eight are the mass grid outer sectors (Figure 4.4(b)).

Angle model outer sectors form the natural cone shape of the direction compass, originating from some choice of centre. The angle model has eight directions with a $45^{\circ}$ angle for each sector respectively, and these gives us the angle outer sectors (Figure 4.4(c)).

Table 4.2 shows various combinations of centre and outer sectors. We ruled out certain combinations of shape/placement/size as in some cases we came across sectors without extent, caused due to the shape of the region. 
Table 4.2 Placement options for centre/outer sector combinations

\begin{tabular}{|lr|c|c|c|}
\hline centre & $\begin{array}{r}\text { outer } \\
\text { acronym }\end{array}$ & $\begin{array}{r}\text { envelope grid } \\
\text { egrid }\end{array}$ & $\begin{array}{c}\text { mass grid } \\
\text { mgrid }\end{array}$ & $\begin{array}{c}\text { angle } \\
\text { angle }\end{array}$ \\
\hline rmc & mass & & $\nabla$ & $\square$ \\
cc & circle & & & $\square$ \\
emc & envbox & $\square$ & & $\square$ \\
mbc & massbox & & $\nabla$ & $\square$ \\
\hline
\end{tabular}

(a)

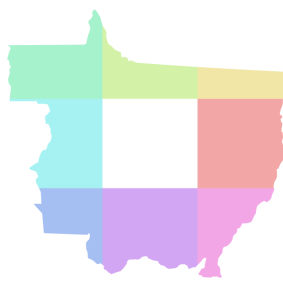

(b)

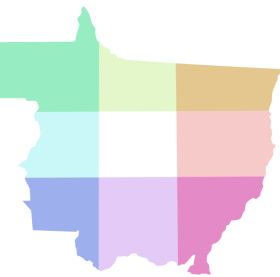

(c)

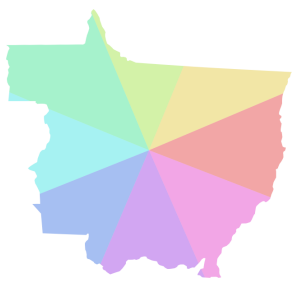

Figure 4.4 Two notions of outer sectors for Mato Grosso state, placed at region mass centre. (a) envelope grid; (b) mass grid; (c) angular sectors.

\subsubsection{Complete models of full directions}

As discussed in Section 4.3.2, full directions consist of cardinal/ordinal and central directions. Each complete spatial extent model for full directions consist of a central sector and some outer sectors. We use intersection of the central sector with the outer sectors, thereby giving us the complete model $\langle$ center $\rangle /\langle$ centralsector $\rangle /\langle$ outersectors $\rangle$. Figure 4.5 illustrates two such complete models. In all, we created 34 different models that we applied to all states in Brazil. The outcomes of model analysis and its evaluation are discussed in Section 4.5.

(a)

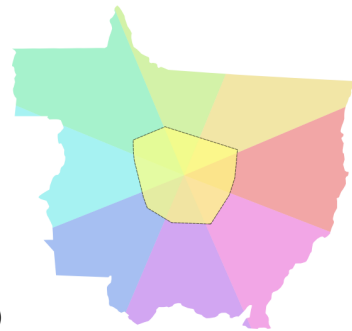

(b)

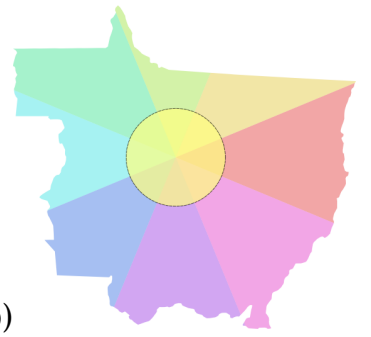

Figure 4.5 Two complete models for Mato Grosso state. (a) mass/hull/angle; (b) circle/circ/angle. 


\subsubsection{Models for half-direction phrases}

\subsubsection{Empirical data analysis}

Our models for half-directions, whether central or non-central, need to express that only a partial area of the underlying outer sector matches the half-direction phrase. While on the one hand, we want to make this partial area big enough to capture and characterize most entries for the phrase, we do, on the other hand, not want to make it overly big, as this would defy the intent of proper spatial characterization.

The approach that we take to this characterization makes use of an entry's distance-to-centre and azimuth-from-centre. Where the latter is a measure that perhaps depends on shape of region and its sectors, but not on their size, the first is size-dependent, and expressions of distance limits for half-direction entries must be relative to some size characteristic of the region or its sectors.

We thus developed a notion of average distance-to-centre over all points in a region, and it is defined in Section 4.6.2.2. This unique distance value for a region serves as a normalizing factor for distances, and we use it as a unit to express the distances-to-centre of half-direction entries. With appropriately chosen lower and upper bounds, this allows us to identify a part of a sector as half-direction characterization.

Our choices of bounds are themselves determined through a distanceto-centre analysis over all entries in both categories of half-direction, using the normalized distance for each region. This empirical study demonstrates that half-directions category ' $\mathrm{c} \star$ ' and ' $\star \mathrm{c}$ ' display a normally distributed normalized distance-to-centre, and we use the characteristics of that distribution to set appropriate symmetric, two-tailed distance limits. The details of both distributions are in Section 4.6.2.2.

\subsubsection{Models for central half-direction phrases}

Central half-directions (' $\star c$ ') are those entries in which the word central is used after the direction name, such as in "northern central Paraná." Once again, we use three important characteristics for model construction: shape, placement and size.

Since half-directions are a subtype of the cardinal directions as shown in Figure 4.2, we use an angle-based approach for constructing the ' $\star c$ ' sectors. For the overall shape of the ' $\star c$ ' model, we use circle, hull, and oval shapes for the central sector. For placement, we use region mass centre, mass box centre and circle centre for positioning the model in the region. Our base model is circle shape as they are equidistant from centre. We construct two circles at maximum and minimum distance from centre for ' $\star c$ ' entries. These distances are different for different confidence intervals around a chosen mean, which themselves are chosen to range from $65 \%$ to $95 \%$. The area bounded by circle shapes represents the area with the accumulated probability of the stated percentage of ' $\star c$ ' entries falling within it. Below, we explain the construction of circle, hull and oval shape models. 
Circle shape We first construct two circles, the smaller circle and the bigger circle, around the centroid of a region. The radii of these circles set distance limits between which the entries are considered to be part of the ' $\star c$ ' half-direction sector. Discs implied by both the circles are intersected with the region, after which the geometric difference of these two results gives the donut shaped circle model. This circular donut area is where the stated percentage of entries are expected to fall. As mentioned earlier, these distances differ for different percentage levels. Hence, to find out these radii, a two-tailed symmetric split of the normal distribution curve was created, for different percentage levels and the radiies were found. The circle donut, thus created is back-intersected with the angle sectors that gives us ' $\star c$ ' circle model as shown in Figure 4.6(a).

Hull shape We first construct a convex hull of the region. Then using our scaling algorithm we scale down this hull twice to the size of smaller and bigger circles above. This gives us the smaller and bigger hull of a size equal to that of the circles, to allow fair comparison. Like with circles, we do the intersection of the hulls with a region, and then the geometric difference of the bigger and smaller hulls, giving the hull-shaped donut model. The hull donut is then back-intersected with the angle sectors to give the ' $\star$ c' circle model as shown in Figure 4.6(b).

Oval shape Oval shapes are constructed using the approach as discussed in Section 4.4.1.1. We construct two ovals and scale them down to the size of smaller and bigger circles above, giving smaller and bigger ovals of a size equal to that of the circles. Like circles and hulls, we intersect the ovals with the region, after which the geometric difference of the bigger and smaller ovals, gives the oval-shaped donut. This donut is back-intersected with angle sectors, giving ‘ $\star c$ ' oval model (Figure 4.6(c)).

Section 4.6.2.2 discusses a detailed analysis of the choices taken in constructing the ' $\star c$ ' models.

(a)

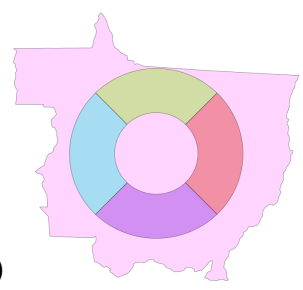

(b)

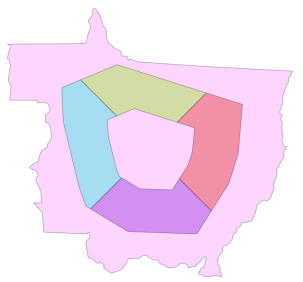

(c)

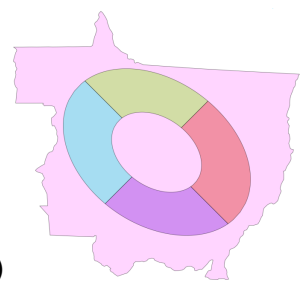

Figure 4.6 Two central half-direction models for Mato Grosso state, placed at region mass centre: (a) circle ' $\star c$ ' ; (b) hull ' $\star c$ ' ; (b) oval ' $\star c$ ' model. 


\subsubsection{Models for non-central half-direction phrases}

The direction phrases that use the word 'central' before the direction name are called non-central half-directions (' $c \star$ '). For example, "central eastern Minas Gerais." The model construction process for ' $c \star$ ' models is similar to that of ' $\star c$ ' models. We apply the same characteristics: shape, placement and size to them.

Here as well, we use circle, hull and oval shapes for construction of ' $c \star$ ' models. The size of the circle model is determined in a similar way as with the ' $\star c$ ' circle model above. The only variation is in the values chosen for minimum and maximum distance, which are derived by varying percentage levels representing the accumulated probability of the ' $c \star$ ' entries on the normal distribution curve. This results in bigger ' $\mathrm{C} \star$ ' circle model than the ' $\star \mathrm{c}$ ' circle model above. This is in line with the expectation that the non-central half-direction entries fall farther away from the centre than the central half-direction entries. Figure 4.7 shows the ' $c \star$ ' circle and hull models. It is important to note that the size of a ' $c \star$ ' hull model is equal to that of the ' $c \star$ ' circle model at the same percentage levels, as determined before back-intersection.

(a)

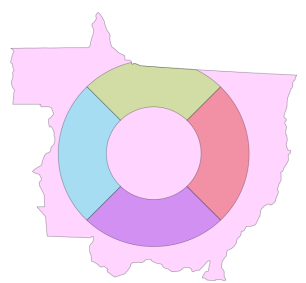

(b)

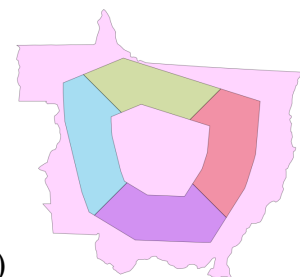

(c)

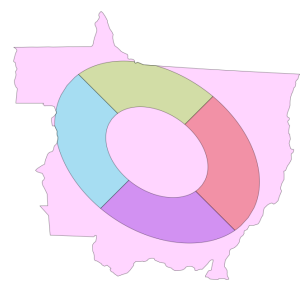

Figure 4.7 Two non-central half-direction models for Mato Grosso state, placed at region mass centre at $65 \%$ level: (a) circle ' $c \star$ ' ; (b) hull ' $c \star$ ' ; (c) oval 'c $\star \star$ ' model.

\subsubsection{Models for extreme direction phrases}

The extreme direction phrases that use the word 'extreme' before the direction name. It is important to note that the word extreme is relative to the direction sector in the region, and should not be interpreted as a single distance from centre for all sectors in the region. For example, "extreme northern Para" may be far away, while at the same time "extreme eastern Pará" may be (much) closer to the centre of the region.

As with other directions, there are three important characteristics of extreme direction models, shape, placement and size. For sectors, we use the angle sectors as detailed in Section 4.4.1.2. For placement, we use region mass centre, massbox centre and circle centre. For size, we use two approaches: distance-based and area-based, which are explained below. 
Distance-based approach gives us eight sectors. In each sector, we calculate the maximum distance-to-centroid for each point on the outer boundary of the sector. Then, we create circles with radii of $65 \%$ of that maximum distance for each sector in the region, giving us eight circles. The geometric difference of respective angle sector and the circle gives the distance-based extreme direction model. Likewise, we create models at varying distance with percentages from 65 to 95-percentage of the maximum distance for each sector. Figure 4.8(a) illustrates one such extreme direction model.

Area-based approach uses different shapes (circle, hull, oval) placed at region mass centre such that the size of the shape ranges from 65 to 95-percentage level of the size of the region. The geometric difference of these shapes (circle/hull/oval) with the region gives us extreme regions which is equivalent to 100 - percentage level. The extreme model, thus created is back-intersected with the angle sectors that gives extreme direction model. Figure 4.8(b) shows a circle shape extreme direction model.

(a)
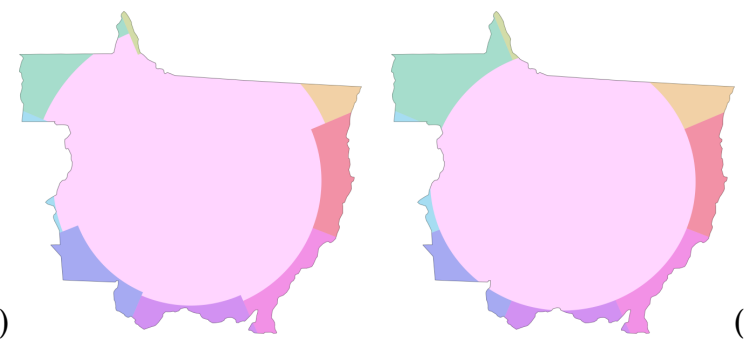

(b)

Figure 4.8 Two extreme direction models for Mato Grosso state, placed at region mass centre. (a) distance-based circle shape at $75 \%$; (b) area-based circle shape at $75 \%$.

Observe that the distance-based approach is naturally restricted to the circle shape, while the area-based approach allows use of different shapes such as circle, hull, and oval.

\subsection{Model performance and comparison}

\subsubsection{Preliminaries}

In the sections below, we study the performance of models from an information retrieval perspective, in full direction, half-direction, and extreme direction models. These models were constructed on a per-state basis for all of the 25 states of Brazil, and we subsequently assessed their performance with the use of all relevant entries in the gazetteer corpus. The corpus holds 1304 full direction entries, 532 half-direction entries, and 152 extreme direction entries. We constructed and evaluated 
34 full-direction spatial extent models, 126 half-direction models, and 95 extreme direction models.

We compare these spatial extent models on the basis of precision/recall scores, as used in information retrieval van Rijsbergen (1979). In context of this work, precision is a percentage measure of how many location entries that fall within a model sector are tagged as being in that sector. This measure is highly influenced by the entry's author cognition and work accuracy, which is a given in the data. Recall is a percentage measure that expresses how many of the entries tagged to be in a sector actually fall inside the sector constructed by the model. This measure is highly influenced by the model construction (position, size, shape), and in a way is more important for our work, as we can influence those choices. While optimally both scores must be high, we typically give preference to recall where we must balance one against the other.

Our spatial extent models apply various parameters (placement/shape/size), each of which comes with a mutually differing number of choices. To methodically evaluate the different model scores, we devised a score matrix per parameter, and a matrix comparison metric that (1) ranks choices per parameter by matrix transposition, and (2) helps to prioritize parameters by pairwise matrix comparison. Our evaluation aims to identify which choice to make for each parameter. The three model parameters of placement, shape and size may, for instance, have two, four and three possible choices. In this hypothetical case, we would thus have a maximum of $(2 \times 4 \times 3=) 24$ different spatial extent models.

For each parameter $p$, we define an $n \times n$ score matrix $M^{p}$, in which $n$ is the number of options for $p$. The matrix value $M^{p}[i, j]$ denotes the number of models with choice $i$ for parameter $p$ that outperform a model with choice $j$, all choices for other parameters of the two compared models being equal. To illustrate this further, Figure 4.9 shows two models at different centers $(n=2)$ but all other parameters, i.e., shape and size being same for central sector and outer sectors in them. So, for both models the shape and size of the central sector is hull and $\frac{1}{9}$ respectively, and for outer sectors shape/size is angles at $45^{\circ}$. Such matrices are anti-diagonal: values for $M^{p}[i, i]$ are always 0 . The resulting matrix is transposed in such a way that rows $i$ are ranked in descending order by $\sum_{j} M[i, j]$, bringing higher values towards the top of the matrix. Observe that the paired difference $M[i, j]-M[j, i]$ is a metric to prefer choice $i$ over choice $j$.

The top row of the transposed $M^{p}$ typically indicates the preferred choice for parameter $p$, but choices for different parameters are not independent from each other, so how do we prioritize the parameters, i.e., how to decide which parameter to evaluate first? We do so by a score metric $\mu$ that exploits the paired difference observation above, and which is defined as

$$
\mu\left(M^{p}\right)=\frac{1}{n-1} \sum_{i<j}\left(M^{p}[i, j]-M^{p}[j, i]\right) .
$$




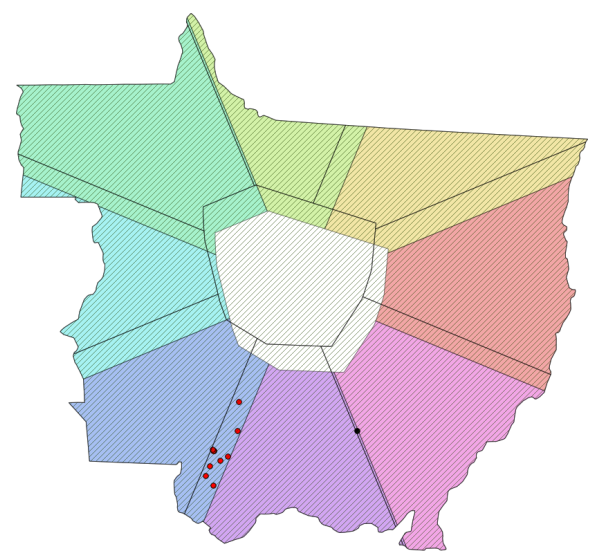

Figure 4.9 Comparison of two full direction models at circle center (hatched) and mass box center (in color) but with same shape and size parameters for central sector and outer sectors. Indicated as black points are entries correctly captured in the hatched model but not in the other; indicated as red points are those correct in the colored model but not in hatched. The color model performs better.

It can be shown easily that $\mu$ is a metric displaying no bias towards the size $n$ of matrix $M$, and this allows us to use it as a comparison metric between such matrices. Observe that a large $\mu$ value indicates a better discrimination between winning and loosing choices encoded with $M$. Comparing between matrices, the larger $\mu$, the higher priority its parameter should be given. We thus rank parameters by descending $\mu$ scores, and examples are found below.

Multiple parameters may be left to prioritize after a top priority parameter (and its choice) has been identified. By a matrix $M^{p \mid q}$ we mean the matrix obtained for parameter $p$, given the choice made for parameter $q$. Where we use this notation, it will be clear from context which value for parameter $q$ is chosen.

For some of our model constructions, we needed a notion of average distance to centroid for a region. It expresses how far, on average, a point $r$ within a region $R$ is to that region's centroid. Theoretically, the definition is

$$
\operatorname{avg} \text { dedist2centroid }(P)=\frac{1}{\operatorname{area}(R)} \int_{r \in R} \operatorname{dist}(\operatorname{centroid}(R), r) \delta r .
$$

We use this metric to normalise distances to centroid within different regions, so as to make them and derived notions such as unit discs around centroid comparable. Details of function implementation can be found in de By (2012). 
In the following sections, we study the performance of models, in full direction models, half-directions models, and extreme direction models.

\subsubsection{Full direction models}

To compare our 48 spatial extent models of interpretation, we computed precision/recall scores van Rijsbergen (1979) for all the models. The 1304 entries tagged to be in a regional sector were used to compute these scores. The resulting recall scores $R$ ranged between 0.50 and 0.59 , while precision scores ranged $P$ between 0.50 and 0.61 .

Score matrices and metrics for the parameters of center placement, central sector shape, and outer sector shape were as follows.

$$
M_{\text {full }}^{\text {center }}=\begin{aligned}
& \text { massbox } \\
& \text { mass } \\
& \text { circle }
\end{aligned}\left[\begin{array}{ccc}
0 & 14 & 8 \\
0 & 0 & 8 \\
0 & 0 & 0
\end{array}\right] \quad M_{\text {full }}^{\text {central }}=\begin{aligned}
& \text { hull } \\
& \text { oval } \\
& \text { circle } \\
& \text { clone } \\
& \text { mbox }
\end{aligned}\left[\begin{array}{lllll}
0 & 6 & 7 & 8 & 5 \\
1 & 0 & 5 & 7 & 5 \\
1 & 2 & 0 & 5 & 5 \\
0 & 1 & 3 & 0 & 5 \\
0 & 0 & 0 & 0 & 0
\end{array}\right]
$$

$$
M_{\text {full }}^{\text {outer }}=\underset{\text { grid }}{\text { angle }}\left[\begin{array}{ll}
0 & 6 \\
0 & 0
\end{array}\right]
$$

Just to illustrate once more, the ' 14 ' in the first matrix (i.e., $\mathrm{M}_{\text {full }}^{\text {center }}$ ) indicates that 14 massbox center models outperform 14 mass center models, all other model parameters being equal. The respective $\mu$ values for these matrices are $15,12.5$, and 6 , indicating that the choice of center deserves highest priority, and that it should be massbox center.

Subsequent determination of $M^{\text {central|center }}$ and $M^{\text {outer|center }}$, with hull as the choice for central sector, continues to identify the massbox center and angular outer sectors as optimal choices.

\subsubsection{Half-direction models}

Using precision and recall scores, all ' $c \star$ ' and ' $\star c$ ' models were compared. All 532 half-direction entries in the gazetteer were used to determine the precision $P$ and recall $R$ score for each model. The results are discussed in detail below.

\subsubsection{Comparison of central models}

We created 63 models for ' $\star c$ ' half-directions based on the choice of centre, shape and seven percentage levels ranging from $65 \%$ to $95 \%$ discussed in Section 4.4.2.2. We found that overall, the $P$ ranges between 0.73 and 0.91 and $R$ range between 0.46 and 0.79 for all ' $\star c$ ' models. An overview of model precision and recall scores is provided through Figure 4.10 . 

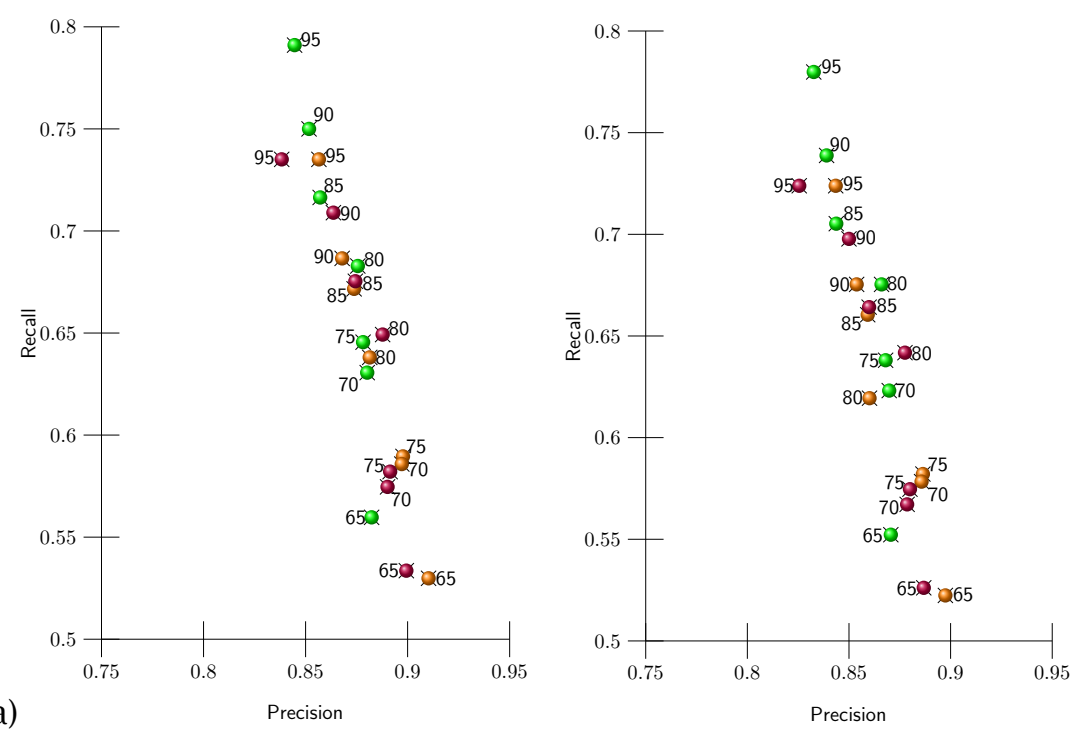

(b)

Figure 4.10 Comparison of performance as precision/recall of 63 ' $\star c$ ' models against gazetteer entries $(n=268)$ with choice of shape circle (green), oval (orange), hull (purple) at (a) region mass center, (b) mass box center.

With respect to recall, we found that models at mass centre in all shapes and at all percentage levels give higher recall scores and are closely followed by models at mbox centre with same shapes and at same percentage levels. When comparing the precision scores, we found that the top three higher precision models were also placed at mass centre. Among the three shapes, the hull models have higher precision $(P=0.91)$ closely followed by oval models $(P=0.89)$ and then circle models $(P=0.88)$. It is difficult to identify whether to choose models on the basis of choice of centre or choice of shape. To overcome this problem we used our prioritisation matrix to choose bet performing models. Score matrices were produced for choices of center and shape, in the understanding that a score matrix for size confidence interval naturally produces an optimal score matrix for highest confidence levels. The matrices found were:

$$
M_{\star c}^{\text {center }}=\begin{aligned}
& \text { mass } \\
& \text { circle }
\end{aligned}\left[\begin{array}{ccc}
0 & 21 & 21 \\
0 & 0 & 21 \\
0 & 0 & 0
\end{array}\right] \quad M_{\star c}^{\text {shape }}=\begin{aligned}
& \text { circle } \\
& \text { oval }
\end{aligned} \text { hull }\left[\begin{array}{ccc}
0 & 18 & 20 \\
3 & 0 & 12 \\
1 & 7 & 0
\end{array}\right]
$$

For score metrics $\mu$, we found that $\mu\left(M_{\star c}^{\text {center }}\right)=31.5$ and $\mu\left(M_{\star c}^{\text {shape }}\right)=$ 19.5, indicating that a choice of mass center deserves priority. We subsequently determine $M_{\star c}^{\text {shape|center }}$ giving: 


$$
M_{\star c}^{\text {shape|center }}=\begin{aligned}
& \text { circle } \\
& \text { oval } \\
& \text { hull }
\end{aligned}\left[\begin{array}{lll}
0 & 7 & 7 \\
0 & 0 & 4 \\
0 & 2 & 0
\end{array}\right]
$$

The conclusion is that the best central half-direction models are those that position their center at mass center, and apply a circular central sector.

\subsubsection{Comparison of non-central models}

Like in ' $\star c$ ' models, we created 63 models for ' $c \star$ ' half-directions. Overall, the $P$ scores range between 0.85 to 0.91 and $R$ scores from 0.55 to 0.85 for all ' $\mathrm{C} \star$ ' models. An overview of model precision and recall scores is provided through Figure 4.11 .

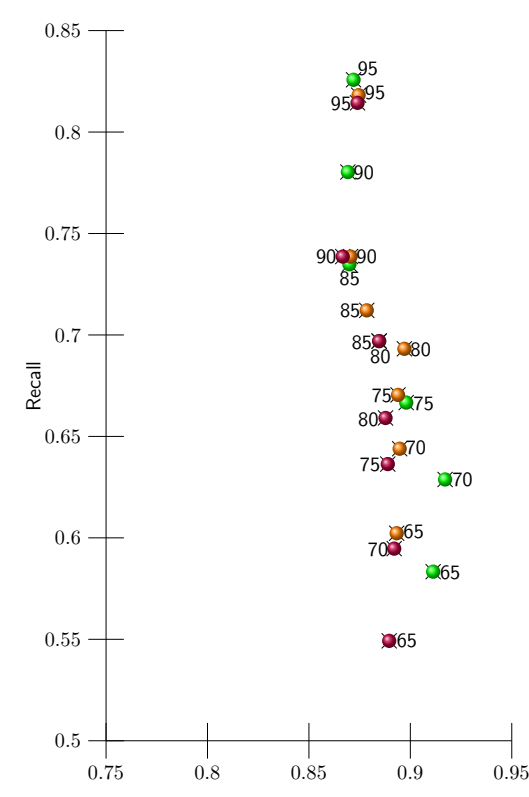

(a)

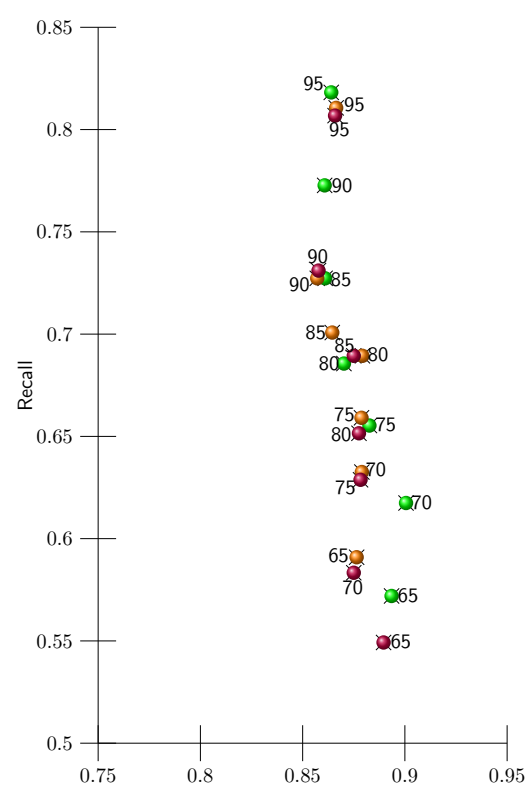

Precision

(b)

Figure 4.11 Comparison of performance as precision/recall of 63 ' $c \star$ ' models against gazetteer entries $(n=264)$ with choice of shape circle (green), oval (orange), hull (purple) at (a) region mass centre, (b) mass box centre.

To identify the best performing ' $c \star$ ' models, we again used the prioritisation matrix discussed in previous section. The matrices for the choice of centre and choice of shape are: 


$$
M_{c \star}^{\text {center }}=\begin{aligned}
& \text { mass } \\
& \text { circle }
\end{aligned}\left[\begin{array}{ccc}
0 & 20 & 20 \\
0 & 0 & 19 \\
1 & 2 & 0
\end{array}\right] \quad M_{c \star}^{\text {shape }}=\begin{aligned}
& \text { circle } \\
& \text { hull } \\
& \text { oval }
\end{aligned}\left[\begin{array}{ccc}
0 & 14 & 21 \\
7 & 0 & 16 \\
0 & 4 & 0
\end{array}\right],
$$

giving rise to the metric values $\mu(M)_{c \star}^{c e n t e r}=28$ and $\mu(M)_{c \star}^{\text {shape }}=20$, prioritizing the choice for circle center. Subsequently, we determined

$$
M_{\mathcal{C} \star}^{\text {shape|center }}=\begin{aligned}
& \text { circle } \\
& \text { hull } \\
& \text { oval }
\end{aligned}\left[\begin{array}{lll}
0 & 4 & 7 \\
3 & 0 & 6 \\
0 & 0 & 0
\end{array}\right]
$$

leading to the conclusion that for non-central half-directions as well, models using mass center and circular central sectors perform best. Findings on size confidence intervals were similar to those for central half-direction models.

\subsubsection{Extreme direction models}

Using the two approaches (Section 4.4.3) we created four model types, one using a distance-based approach (mass/circle/angle) and three using an area-based approach (mass/circle/angle, mass/hull/angle, mass/oval/angle), models were constructed in each approach with percentages ranging from $5 \%$ to $40 \%$ levels (discussed in Section 4.4.3). The resulting 95 spatial extent models were evaluated with 152 corpus entries. Precision values ranged between 0.52 and 0.75 , and recall values ranged between 0.07 and 0.63 . The latter variability was entirely caused by a wide range of size parameter values. An overview of model precision and recall scores is provided through Figure 4.12.

The score matrices found for extreme direction models were as follows:

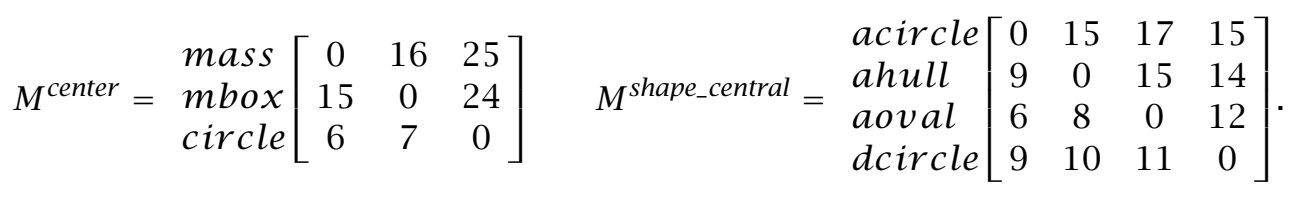

These matrices give us $\mu(M)^{\text {center }}=18.5$ and $\mu(M)^{\text {shape_central }}=11.7$, prioritizing the choice for mass center. We subsequently determined the conditional matrix for choice of central sector, given the mass center 

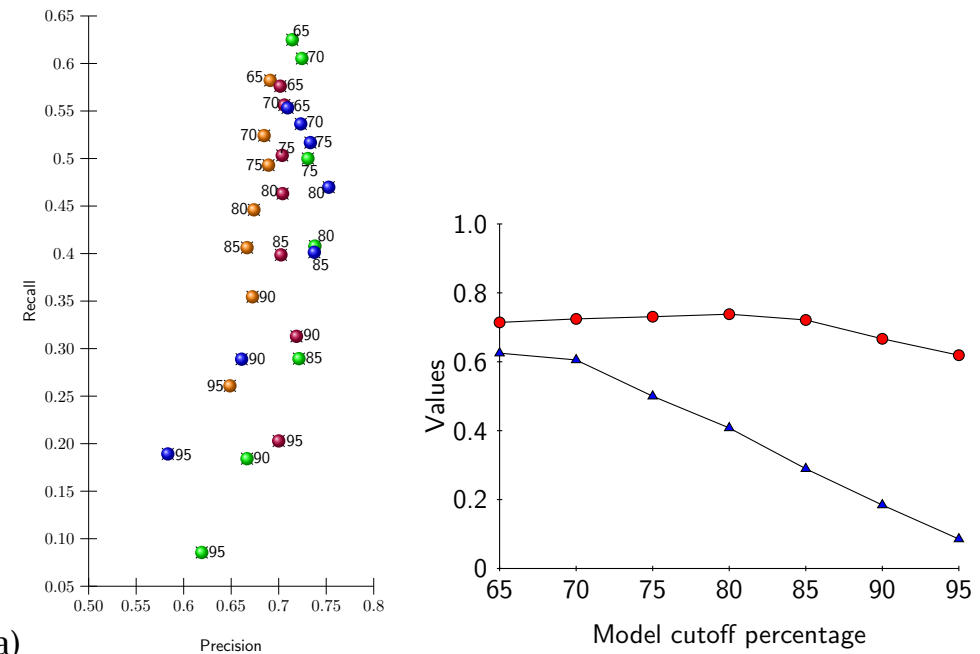

(b)

Figure 4.12 (a) Comparison of performance as precision/recall of 95 models against gazetteer entries $(n=152)$ with choice of shape dist/circle (green), area/circle (orange), area/oval (blue), area/hull (purple) at mass centre, (b) Trend of precision (red) and recall (blue) for dist/circle model at mass centre.

pick:

$$
M^{\text {shape_central|center }}=\begin{aligned}
& \text { acircle } \\
& \text { ahull } \\
& \text { aoval } \\
& \text { dcircle }
\end{aligned}\left[\begin{array}{llll}
0 & 5 & 5 & 4 \\
3 & 0 & 5 & 5 \\
3 & 3 & 0 & 5 \\
4 & 3 & 3 & 0
\end{array}\right],
$$

to find that we should opt for minimal bounding circle centers. Observe however that the $\mu$ value of 3.3 for this matrix is low, indicating only subtle differences between candidate choices.

\subsection{Data analyses}

\subsubsection{Centre of variable position for full directions}

The choice of centre affects the azimuth of entries from centre. For full directions, we studied this impact for all eight directions and all four notions of centre. To do so, we created boxplots (Abuzaid et al., 2011) with 10/25/50/75/90-percentiles for deviation from the main direction. All four cases, are provided in Figure 4.13.

We find that the two mass-based centres, i.e., region mass centre (a), and mass box centre (d) perform very similarly. The pair of bounding box-based centres, i.e., envelope mass centre (b), and circle centre (c) also perform quite like each other. The latter two display areas of overlap 
(a)

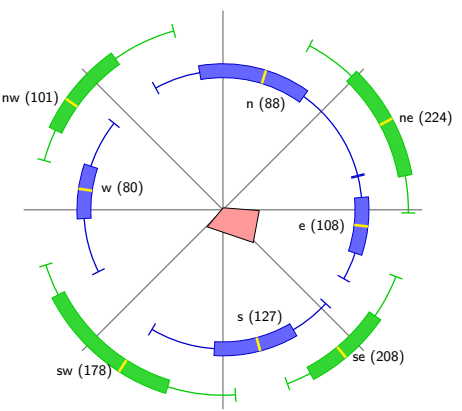

(c)

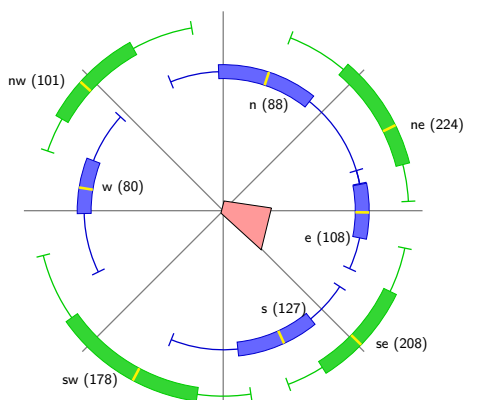

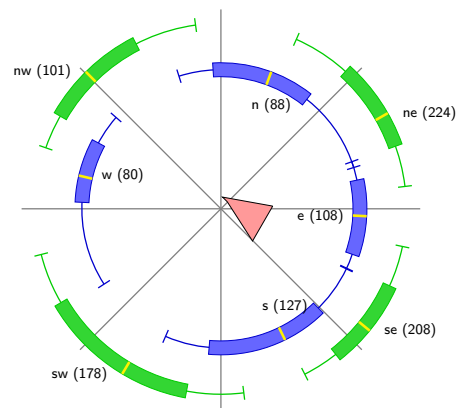

(b)

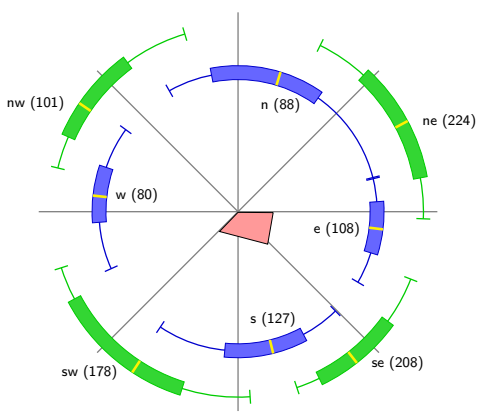

(d)

Figure 4.13 Azimuth spread for the full directions (cardinal in blue and ordinal in green), indicated as 10/25/50/75/90\% boxplots over the compass rose for four notions of centre: (a): region mass centre, (b): envelope mass centre, (c): circle mass centre, (d): mass box centre. In red: area of predicted optimal centre.

between cardinal and ordinal directions, and hence have lower model performance. The eastern skew of the data also creates a tendency in all models to 'hang towards east'. This is illustrated by the red areas in the graph above, which indicate the areas of predicted optional centre. It needs to be mentioned that since in our dataset, ordinal direction entries are more common than cardinal direction entries, we have considered wider angles around ordinals at the cost of cardinal directions. This has been detailed in Section 4.6.2.

\subsubsection{Sectors of variable size}

\subsubsection{Full directions}

Central sectors of variable size: In our dataset, an average of 140 entries exists for each of the eight compass directions. Interestingly, the 'central' entries are a total of 190 and this warrants a formation of a separate ninth group. Hence, our axiomatic $\kappa=\frac{1}{9}$ rule for the central sector seems natural. One may consider that choice arbitrary if not studied further. We therefore study the impact of 
varying size of central sector on model performance. We find that with limits, a bigger central sector improves model performance.

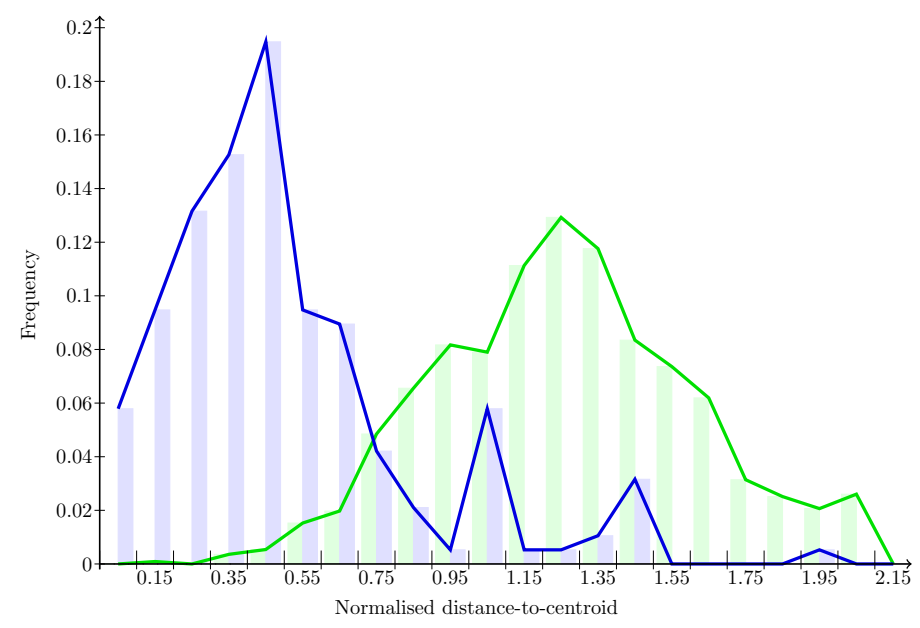

Figure 4.14 Frequency of normalised distance-to-centroid of full direction entries: (a): central (blue), (b): non-central entries (green) in the study.

We picked our best performing model mass/hull/angle, and optimised it against the data by varying the value of factor $\kappa$ for size of central sector. We find that its performance gradually increases to an optimal level at $\kappa=0.177$, meaning $\rho \approx 0.434$. With that factor there is a $5 \%$ better precision/recall score for the model for our dataset, as illustrated by Figure 4.15(a).

In search of an alternative corroboration, we devised a metric named avg_dist2centroid, per region $P$, defined as

$$
\operatorname{avg} \text { _dist2centroid }(P)=\frac{1}{\operatorname{area}(P)} \int_{p \in P} \operatorname{dist}(\operatorname{centroid}(P), p) \delta p .
$$

The metric determines the average distance to centroid over all points within a region $P$. We use this metric to normalise distances to centroid within different regions, so as to make them comparable (de By, 2012). We analysed the distribution of all normalised distances-to-centroid, for both central and non-central entries. From the two histograms (Figure 4.14), probability density functions are derived by fitting a standard normal distribution with the sample of non-central entries, giving $N[\mu=1.2628 ; \sigma=$ $0.3558 ; n=1114]$, and a Rayleigh distribution for the central entries, giving $R[\hat{\sigma}=0.4234 ; n=190]$.

The intersection point of distributions $N$ and $R$ lies at the normalised distance of 0.8470 , and marks the distance at which entries can be assigned with equally strong evidence to either the central 
sector or an outer sector. It defines an appropriate average distance of the central sector boundary to the region's centroid. Taking the argument a little further, if it would be a circle central sector, the normalised distance value found above translates to $\kappa$ values around 0.17 for larger states, and towards 0.30 for smaller states in our study. In other words, an optimal choice for $\kappa$ depends on size of the region.

Independently of these finds, we ran three of our best performing standard models with a range of $\kappa$ values, varying between 0.10 and 0.40. That model ran against our current dataset displayed optimal performance at $\kappa=0.19$ (giving $P=0.64$ ), well above our start position of $\kappa=0.11$.

(a)
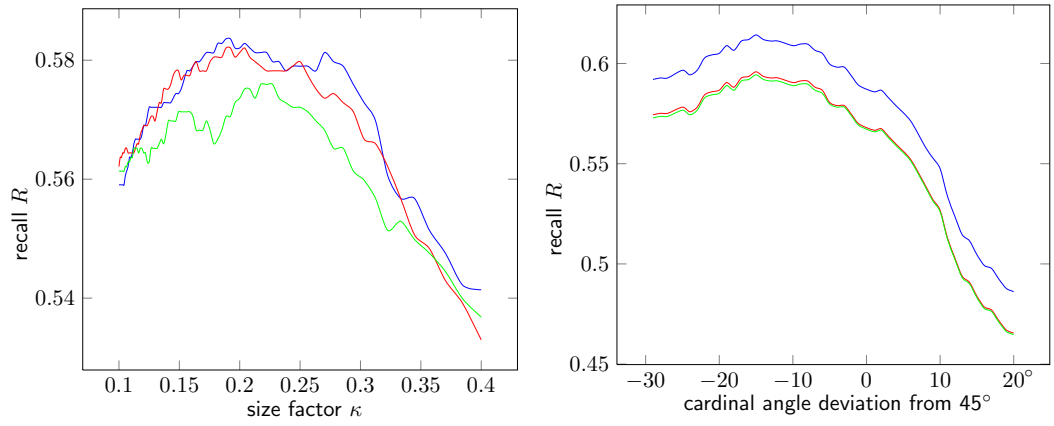

Figure 4.15 Optimisation of three models against size of central and angle of cardinals. (a): recall for three models with varying values for size factor $\kappa$; (b): recall with varying values for deviation from the $45^{\circ}$ angle for cardinal directions. mass/hull/angle (blue), mass/oval/angle (red), mass/clone/angle (green).

Outer sectors of variable size: Since it has already been established that angle outer sectors outperform other outer sectors, we also studied variation in the angle sizes associated with different compass directions. We initially expected models to do better with larger angles for cardinal directions, but we find opposite results. Keeping in mind the frequencies of cardinal and ordinal directions provided in Section 4.1, and the measures of angle deviation around the eight compass directions of Figure 4.13, we believe it is worth considering variable angle size per direction.

Hence, we varied the angles for ordinal directions by making them larger at the expense of those for cardinal directions. Angular spread around the mean per direction was essentially the same for both groups of directions. The data however, appeared to indicate that cardinal directions display a somewhat bigger angular spread $\left(\sigma_{\text {card }}=34^{\circ} ; \sigma_{\text {ord }}=27^{\circ}\right.$ when outliers were included, and $\sigma_{\text {card }}=25^{\circ} ; \sigma_{\text {ord }}=25^{\circ}$ when without outliers).

In spite of it all our models performed better, with wider angles associated with ordinal outer sectors. In fact, a $60^{\circ}$ wide spread 
for ordinals versus $30^{\circ}$ for cardinals came out as the best choice. Figure 4.15 illustrates the performance of three models on our dataset. This is due to the higher frequency of ordinal direction entries over cardinal direction entries.

\subsubsection{Half-directions}

Distance analysis: We discussed various placement centres in Section 4.6.1, based on which we carry out further analyses and model creation for half-directions. In our corpus, we have 25 regions (states) in Brazil and as can be expected, they are substantially different from each other in size. This makes the comparison of regions and derivation of generic rules of size and delineation of a central sector per region also challenging. So, to overcome this challenge, we use the same distance metric discussed in Section 4.6.2.1 to normalise the distance-to-centroid within different regions, to make them comparable (de By, 2012). We analysed the distribution of all normalised distances-to-centroid, for both ' $\star c$ ' and ' $c \star$ ' half-direction entries. It was our initial hypothesis that ' $c \star$ ' (e.g., central eastern) and ' $\star c$ ' (e.g., eastern central) entries are separate cases and that ' $\star c$ ' entries occur within the central sector and ' $c \star$ ' entries should be central to the ' $\star$ ' border sector. This is the reason for our separation of central and non-central half-direction entries. To test our hypothesis, we analysed the distribution of normalised distanceto-centroid for ' $\star c$ ' and ' $\mathrm{c} \star$ ' entries by analysing and comparing their histograms and probability density functions (Figure 4.16). Fitting the standard normal distribution curves for the ' $c \star$ ' entries, we derive $N[\mu=0.8539 ; \sigma=0.3049 ; n=264]$ and for ' $\star c$ ' entries gives $N[\mu=0.7436 ; \sigma=0.2850 ; n=268]$. Hence, $\mu$ and $\sigma$ are calculated with the respective regions average normalised distance as unit. This revealed that the $\mu$ values (or avg_dist2centroid) for ' $\star c$ ' and ' $c \star$ ' entries are rather close at 0.74 and 0.85 normalised unit distance, respectively. This should not have been the case if our hypothesis was true. The difference between these $\mu$ values should have been greater and the amount of overlap should also have been smaller. We also found that the intersection point of ' $\star c$ ' and ' $c \star$ ' distributions lies at the normalised distance of 0.83 which is close to the intersection point $N$ and $R$ distribution of full directions, i.e., 0.847 normalised distance. This provides strong evidence that indeed our initial hypothesis above is true. Here, we took our test of hypothesis to the next stage.

We applied two independent sample t-tests on the normalised distances on the groups of ' $\mathrm{c} \star$ ' and ' $\star \mathrm{c}$ ' entries. The null hypothesis for this test is:

$H_{O}$ : The mean of normalized distances to centroid is equal for both samples of the group, i.e., $\mu_{1}=\mu_{2}$. 


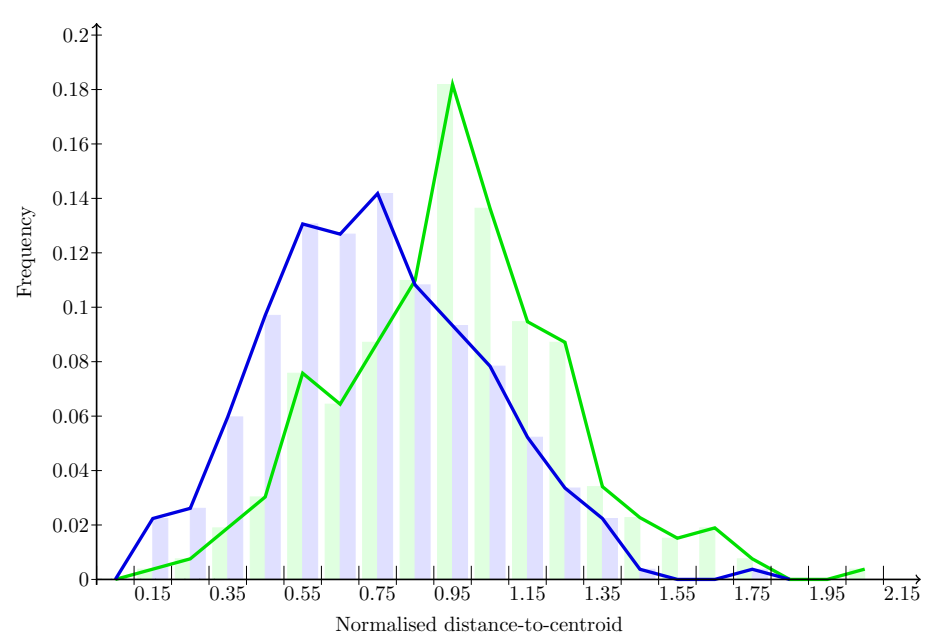

Figure 4.16 Frequency of normalised distance-to-centroid for central halfdirection (blue) and non-central half-direction (green) entries in the study.

We use four groups, reflecting cardinal compass directions, with two independent samples each to test our null hypothesis. The test is done with confidence of $95 \%$ and significance value of 0.05 . The results of each group is reported in Table 4.3 suggesting that our null hypothesis stay valid for only for two groups. As the test is still inconclusive, there is still room for more statistical analyses to test our hypothesis.

Table 4.3 Results of independent sample t-test with unequal variance on halfdirection entries based on normalized distance to centroid

\begin{tabular}{|c|c|c|c|l|}
\hline Notation & nccn & ecce & sccs & wccw \\
\hline Sample 1 & cn & ce & cs & cW \\
\hline $\mathbf{n}_{1}$ & 53 & 101 & 45 & 65 \\
\hline$\mu_{1}$ & 0.848 & 0.903 & 0.871 & 0.771 \\
\hline Sample 2 & nc & ec & sc & wc \\
\hline $\mathbf{n}_{2}$ & 65 & 50 & 120 & 33 \\
\hline$\mu_{2}$ & 0.761 & 0.739 & 0.740 & 0.732 \\
\hline$t$ & 1.374 & -3.704 & 2.862 & -0.583 \\
\hline$d f$ & 108.7 & 118.2 & 81.7 & 62.2 \\
\hline$p$ sig.(1-tailed) & 0.086 & 0.001 & 0.002 & 0.281 \\
\hline Results $\mathbf{( H}_{\boldsymbol{o}}$ ) & valid & rejected & rejected & valid \\
\hline
\end{tabular}

As our next step, we tested if there is any correlation between normalised distances from centroid of all half-direction entries in a region and the region sizes. This test gave a negative result since the correlation coefficient $R^{2}$ was 0.225 for smaller regions and 0.164 for larger regions. 
The correlation between normalised distances from region boundary and region sizes was also tested and the results were negative as $R^{2}=0.08$ was found for smaller regions and 0.004 for larger regions.

Azimuth analysis: We analysed angle spread for ' $\star c$ ' and ' $c \star$ ' entries in $\star$ direction. To do so, we calculated the azimuth of entries from mass region mass centre and azimuth difference for each entry from their respective cardinal direction. The azimuth difference of 'cn' and 'nc' entries, for example, was calculated from the ' $n$ ' direction. After a comprehensive analysis and percentile calculations, circular box plots (Abuzaid et al., 2011) were developed for all eight half-directions. The spread of all entries for $10 / 25 / 50 / 75 / 90$-percentiles as deviation from the main direction as shown in Figure 4.17. From the figure we infer that barring a few exceptions in 'nc, sc, cs' half-directions, entries typically fall within the boundaries of ordinal directions. We use this calculation for model construction in Section 4.4.2. We again tested our hypotheses using the azimuth analysis to see if the ' $\star c$ ' and ' $c \star$ ' entries are different sets.

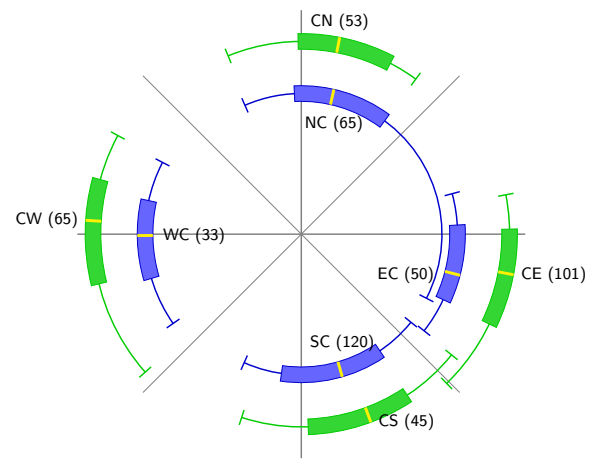

Figure 4.17 Azimuth spread indicated as 10/25/50/75/90\% boxplots from region mass centre over the compass rose for: for individual ' $\star c$ ' and ' $c \star$ ' entries.

A plot of polar coordinates of all half-direction entries using their azimuths from centroid was created and is shown in Figure 4.18.

We expected, while plotting the coordinates on $x-y$ plane, that ' $\mathrm{c} \star$ ' entries fall away from the centroid and ' $\star \mathrm{c}$ ' entries closer to it if they are two different sets. However, it was interesting to see that both sets of entries showed considerable overlap and were quite close to each other, as is illustrated in Figure 4.18.

We applied t-test on the azimuth difference of all half-direction entries using null hypothesis:

$H_{O}$ : The means of azimuth difference to centroid are equal for both samples of the group, i.e., $\mu_{1}=\mu_{2}$. 


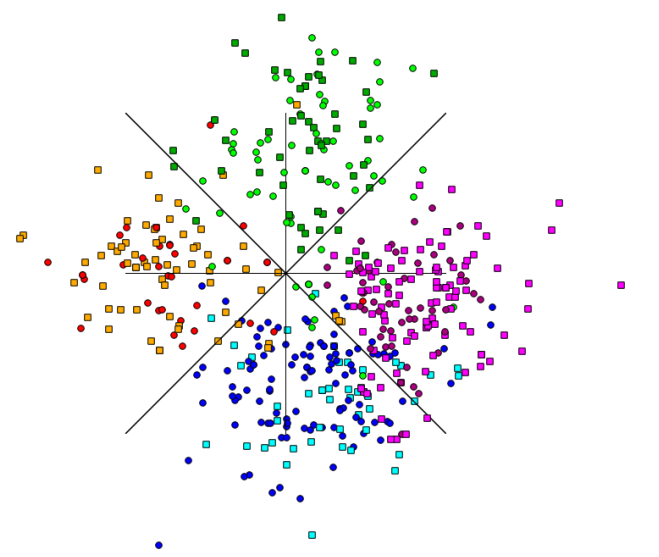

Figure 4.18 All half-direction entries shown in polar coordinates. Entries shown as boxes represent ' $c \star$ ' entries and with circles represent ' $\star c$ ' entries.

Table 4.4 Results of independent sample t-test with unequal variance on halfdirection entries based on azimuth differences

\begin{tabular}{|c|c|c|c|l|}
\hline Notation & nccn & ecce & sccs & wccw \\
\hline Sample 1 & cn & ce & cs & cW \\
\hline $\mathbf{n}_{1}$ & 53 & 101 & 45 & 65 \\
\hline$\mu_{1}$ & -11.343 & -13.292 & 17.740 & 5.329 \\
\hline Sample 2 & nc & ec & sc & Wc \\
\hline $\mathbf{n}_{2}$ & 65 & 50 & 120 & 33 \\
\hline$\mu_{2}$ & -25.026 & -11.735 & 12.054 & 6.367 \\
\hline$t$ & -1.865 & 0.431 & -1.179 & -0.124 \\
\hline$d f$ & 99.187 & 98.565 & 93.617 & 66.236 \\
\hline$p$ sig.(1-tailed) & 0.033 & 0.334 & 0.121 & 0.451 \\
\hline Results (H $\left.\mathbf{H}_{\boldsymbol{o}}\right)$ & valid & valid & valid & valid \\
\hline
\end{tabular}

As per results (Table 4.4) of this test, our null hypothesis is validated for all the four groups i.e., nccn, ecce, sccs, wccw. The circular boxplot of 10/25/50/75/90-percentiles for deviation from the main direction is shown in Figure 4.19.

The discussion around Section 4.6.1, on the e/s/se directional skew is also observed in half-direction entries. Of all 25 states in Brazil, eleven are coastal states and account for $61 \%$ of half-direction entries. We studied this directional skew by dividing the states into two groups: coastal and non-coastal states, based on their location on the e/s/se coast. A comprehensive percentile calculation was again carried out for four directional groups (nccn, ecce, sccs, wccw) separately in coastal and non-coastal states. Figure 4.20(a) shows that coastal states have an evident bias towards e/s/se directions 


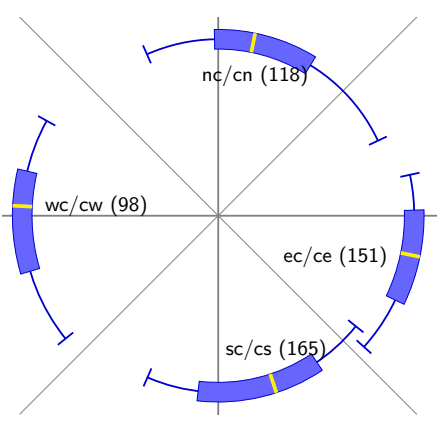

Figure 4.19 Azimuth spread indicated as 10/25/50/75/90\% boxplots over the compass rose for nccn, ecce, sccs, wccw.

(a)
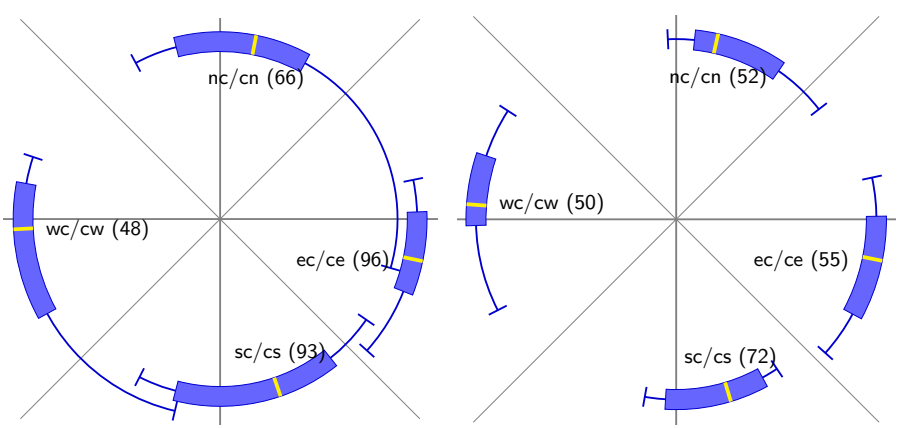

(b)

Figure 4.20 Azimuth spread indicated as 10/25/50/75/90\% boxplots over the compass rose for nccn, ecce, sccs, wccw: (a): for coastal states; (b): for non-coastal states.

supporting our observation above, whereas non-coastal states do not show any skew and have the entries nicely spread in all four directions (Figure 4.20(b)).

\subsubsection{Extreme directions}

Distance analysis To study the distance of extreme direction entries from centroid we calculated the normalised distance-to-centroid for all extreme direction. Once again, we use the distance metric (Section 4.6.2.1) to calculate these normalised distances from centroid. We analysed the histogram and derived the probability density function (Figure 4.21) by fitting the standard normal distribution with the extreme direction entries, giving $N[\sigma=0.3706 ; \mu=$ $1.5577 ; n=152$ ]. We observe that the mean normalised distance of extreme directions ( $\mu=1.5577)$ is farther away from that of noncentral full directions ( $\mu=1.2628$ ) thereby supporting our assumption (Section 4.3.2) that extreme directions should fall closer to the outer border of the sector. This also confirms the need to treat these entries as a different category of directions. 


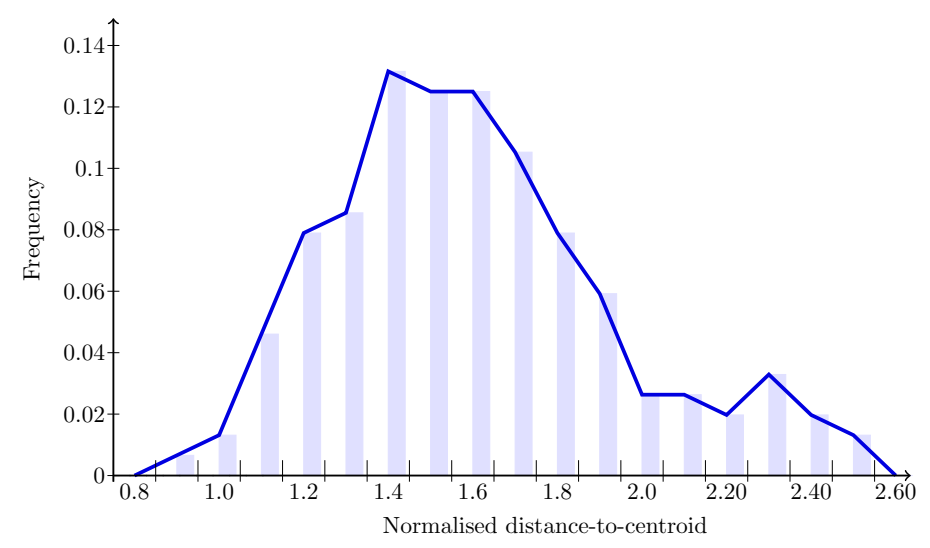

Figure 4.21 Frequency of normalised distance-to-centroid for extreme direction entries.

To check our data for normality we use a Kolmogorov-Smirnov test, which suggest that the data indeed displays a normal distribution. We also used '68-95-99.7' rule of normal distribution Moore et al. (2012) which suggests that, 68-percentage of total extreme direction entries can be expected to fall within $\sigma$ of the $\mu$. Since the $\sigma$ is very small it can be inferred that all the entries are concentrated near the $\mu$ and are not spread out. This could be a possible reason for a sudden drop in the performance of the models from 70-percentage level onwards (Section 4.5.4). It is possible that the $\mu$ distance falls within the range of 60 to 70-percentage level for most regions and hence results in best recall scores for these values.

Azimuth spread Like with full directions and half-direction we calculated the azimuth of entries from region mass centre with their respective cardinal/ordinal directions. The circular box plots were developed for all eight extreme directions for 10/25/50/75/90percentiles as deviation from respective direction angles Figure 4.22. We infer from the figure that all cardinal/ordinal entries show minimal deviation from their respective main angle. However, we observed that two of four ordinal directions i.e., 'xne' and 'xse' show a substantial deviation from their main angles compared to others.

\subsection{Summary}

Directional containment phrases are the most frequently occurring (54\%) spatial hints in our gazetteer descriptions. In this chapter, we created spatial extents models that help to interpret directional containment phrases for each category of direction types, i.e., full directions, half-directions, and extreme directions. We looked at the role played by various charac- 
(a)

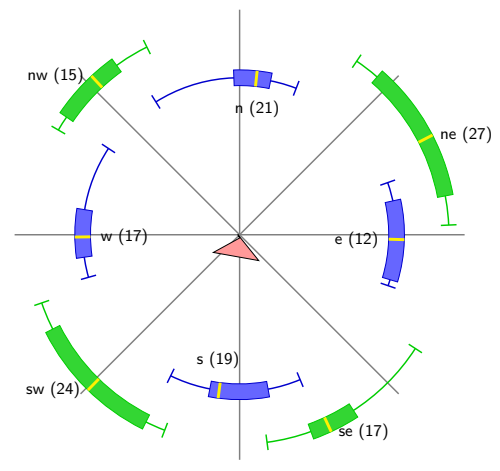

Figure 4.22 Azimuth spread for extreme directions (cardinal in blue and ordinal in green), indicated as 10/25/50/75/90\% boxplots over the compass rose at region mass centre.

teristics, i.e., placement, shape, size in developing spatial extent models. For full directions, different combinations of $\langle$ center $\rangle /\langle$ centralsector $\rangle$ / 〈outersectors〉 give us complete spatial extent models for their interpretation. Apart form various characteristics we also studied the role of average distance-to-centre and azimuth of entries in the region. These were especially helpful in deciding the size of the central sector and of the angular outer sectors. The average distance-to-centroid plays significant role in constructing the models for half-directions and extreme directions.

A significant number of entries for coastal states were found to fall in east/southeast/south part of the states. This directional bias can be understood from the fact that entries represent historic collection sites in Brazil and many expeditions started from the east coast, and such sites are common in the corpus. For instance, we found a large number of entries described as in south of the state but were actually on the south of the coast. Indeed, a study of entries in non-coastal states revealed no such directional bias at all.

Since spatial extent models help in mapping descriptions and to verify description consistency, we evaluated our models on performance, using notions of recall and precision. We found that for full directions, spatial extent modes with 'mass box centre/hull central sector/angular outer sector' is the optimal choice. For both half-directions, 'region mass centre/circle central/angular outer sector' is the optimal choice. For extreme directions, 'region mass centre/area-based circle' approach with angular outer sector is the optimal choice.

Since each description in our gazetteer contains more than one hint, it is important to also look at other hint types in the descriptions. Having spatial representations of more than one hint will be useful in crossvalidating them all and in the end, derive a most plausible estimate of the actual location's coordinates. Thus, in the next chapter, we create spatial representations of other hint types, taking into consideration the 
uncertainty attached to those hints and derive areas of certainty where the locality could be located. 


\section{Spatial representation of hints using probability rasters}

\subsection{Introduction}

Places have always been of interest to humans, and play a fundamental role in day-to-day communications. Often places are described with reference to other places using some spatial relation expressed in natural language, which represents the cognitive conceptualisation of the reporting person. Since neither human cognition nor natural language is free from ambiguity (Longley et al., 2005), it often causes uncertainty in the description.

Uncertainty arises when the available information is not precise. It is important to assess and consider uncertainty while doing spatial analysis or computations because without it, such analysis is of limited use (Fisher, 1999). This has been shown by Rowe (2005), who obtained misleading results while studying the impact of species distribution when uncertainty associated with localities was not considered. Since our gazetteer is a natural language text, many of its spatial hints come with uncertainty. A study conducted by Wieczorek et al. (2004) provides insight into different types of uncertainty associated with textual descriptions in the collection of a natural history museum and it provides methods to address them.

In this chapter, we aim to understand the various uncertainties associated with spatial hints, and to incorporate spatial representations using probability-based raster approaches for such hints. We then wish to computationally combine these probability rasters to derive an area with associated uncertainty for the location of the target locality. These areas with associated uncertainty will also be overlaid with the vector models that we created in previous chapter and the results will be evaluated.

In the following section, we discuss a theoretical framework of uncertainty and in Section 5.3 we discuss related work. In Section 5.4, we discuss various factors that lead to uncertainty and how they can be modeled. In Section 5.5, we discuss the general approach used for creating rasters of probability distribution. In Section 5.6, we discuss raster creation based on parameters derived from different hint types, 
while in Section 5.7, we discuss optimisation of these parameters. In Section 5.8, we overlay the rasters of different hints in a sample of entries to identify common areas with associated uncertainty. These areas are then intersected with the vector models in Section 5.9 and in Section 5.10, we conclude the chapter.

\subsection{Background on uncertainty}

\subsubsection{General definitions}

The limitations in measuring any observable quantity accurately, causes uncertainty in data. The presence of uncertainty in data leads to incorrect predictions that can be fatal and can lead to lowered confidence in the system or the operator (Fisher, 1999). When discussing uncertainty, different terms are frequently used and sometimes considered as components of uncertainty. They are accuracy, precision, error, vagueness, and ambiguity. Accuracy is related to how close the measured value is to its true value or accepted value. Precision is defined as how close are the measured values for a quantity under the same conditions when the same thing is measured several times. Error is defined as the presence of known inaccuracy or problem in the data (Gahegan and Ehlers, 2000; Hunter and Goodchild, 1993). Vagueness occurs when an object is poorly defined, e.g., 'the church is near the river', where the notion of near is subjective. Finally, ambiguity can refer to a situation in which an object can be defined clearly but cannot be placed without doubt in a single class (Comber et al., 2006). There are various theoretical methods to model these types of uncertainty, such as probability theory, fuzzy set theory, and rough sets as shown in Figure 5.1.

In Figure 5.1, we see that when the object is well-defined, the only type of uncertainty can be an error', as could be the case in state boundaries. Vagueness and ambiguity arise when the object is poorly defined. For example, vagueness comes when there is no crisp definition of an object like a hill or mountain and river or stream. Ambiguity can be divided into two parts, discord and non-specificity. Discord arises when there is lack of agreement on the classification of the object. This can be resolved by expert opinion and applying Dempster Schafer's theory (Zadeh, 1986). Similarly, non-specificity comes when an object is assigned to a class which is open to interpretation. For example, in our gazetteer, 'S of Rio de Janeiro' in reality may be interpreted as SE or SW of Rio de Janeiro.

All components of uncertainty in Figure 5.1 are influenced by two main causes, i.e., conceptualisation and measurement. Conceptualisation is attributed to the process of translating real-world entities into map objects and measurement leads to 'imprecision in the measurement' of an entity. The effect of these two causes on uncertainty can be seen in Figure 5.2.

As mentioned above, there are various approaches to model uncertainty. In this research project, we have chosen probability theory and 


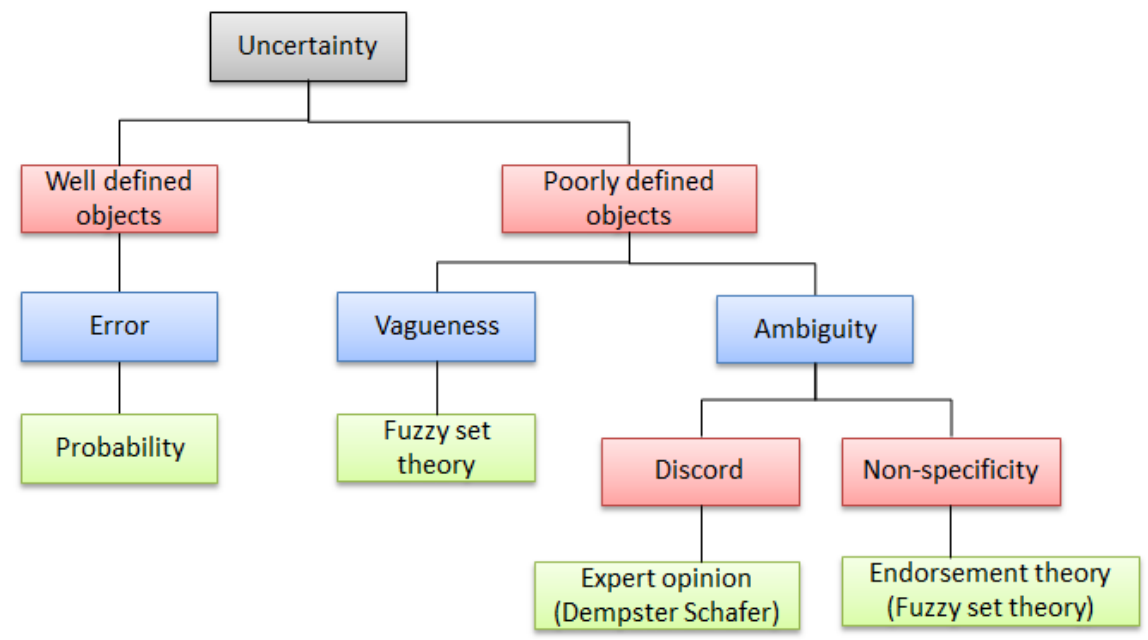

Figure 5.1 A conceptual model of uncertainty in spatial data and methods to handle various types of uncertainty (Comber et al., 2006; Fisher et al., 2006)

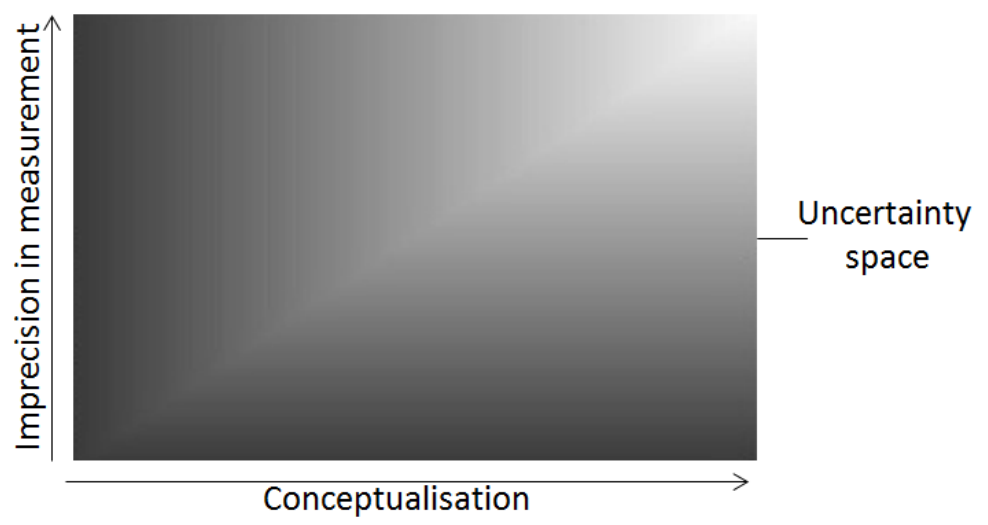

Figure 5.2 The effect on uncertainty by 'measurement' and 'conceptualisation' process. The lighter the space, higher the uncertainty (adapted from (Voudouris et al., 2006))

model uncertainty by it, since probability theory is commonly used for handling uncertainty in geographic entities (Goodchild and Zhang, 2002).

In probability theory, the probability of occurrence of a phenomenon is estimated, e.g., in weather and flood forecasting (Fisher, 1999). These phenomena can be considered as random continuous phenomena, where continuous phenomena are those for which events can occur in continuous space. The probability distribution a random continuous variable can be defined using a probability density function (pdf). Let there be a continuous random phenomena represented by variate $X$. The probability density function (pdf) of $X$ can be represented as $f(x)$ and 
the probability of $x$ falling between an interval $a, b$ can be calculated as: $P(a \leq x \leq b)=\int_{b}^{a} f(x) d x$. When the range is between $-\infty$ and $\infty$, the probability is equal to 1 , i.e., $\int_{-\infty}^{\infty} f(x) d x=1$. There are various pdfs, such as Gaussian distribution, Cauchy distribution and von Mises distribution. In the following sections, we discuss those pdfs that were used in this project.

\subsubsection{Gaussian distribution}

The Gaussian or normal distribution is a commonly used probability model (Moore et al., 2012). The Gaussian pdf to calculate the probability density at any given point $x$ in normal distribution, is as follows:

$$
f(x)=\frac{1}{\sqrt{\left(2 \pi \sigma^{2}\right)}} e^{\frac{-(x-\mu)^{2}}{2 \sigma^{2}}},
$$

where $\mu$ is the mean of data and $\sigma$ is standard deviation. The graph of $f(x)$, as shown in Figure 5.3, is often referred to as density curve and the area under this density curve must be 1 .

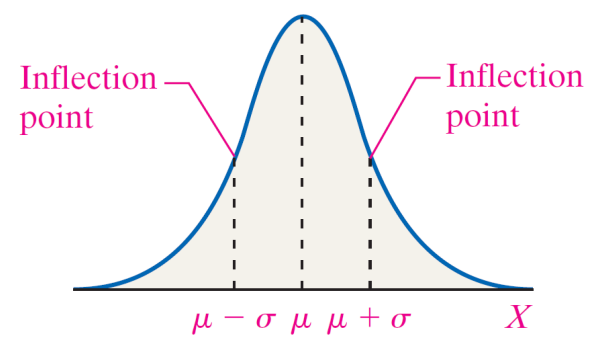

Figure 5.3 The Normal distribution at different $\sigma$ values (adopted from (Sullivan, 2008))

The uncertainty attached to distance information in our hints can be modeled using Gaussian pdf because it behaves similarly to Gaussian distribution. We expect the probability density to be highest at a mean point and as we go away from this mean point, the probability densities are also expected to reduce in continuous manner, like that of a Gaussian distribution. Liu et al. (2009) also mentioned that uncertainty in distance measurement can be considered to be normally distributed.

\subsubsection{Von Mises distribution}

The von Mises distribution is a circular probability model (Gaile and Burt, 1980) and is considered a close approximation of normal distribution. It can be used to model probability of directional information. For example, wind current modeling and modeling geomagnetism require a directional model (Dhillon and Sra, 2003; Mardia and Jupp, 2000). The von Mises distribution uses two parameters: a mean direction $\mu$ and a concentration 
parameter $\kappa$ (Gaile and Burt, 1980). The von Mises pdf for computing probability density for a direction at an angle $\theta$ is as follows:

$$
f(\theta \mid \mu, \kappa)=\frac{e^{\kappa \cos ^{(\theta-\mu)}}}{2 \pi I_{0}(\kappa)},
$$

such that $0 \leq \theta \leq 2 \pi ; 0<\mu<2 \pi ; \kappa>0$, and where

$$
I_{0}(\kappa)=\sum_{r=0}^{\infty}\left(\frac{1}{r}\right)^{2}\left(\frac{\kappa}{2}\right)^{2 r} .
$$

In the Function 5.2, $\mu$ is the mean direction and $I_{0}(\kappa)$ is a particular function of $\kappa$ called the modified Bessel function of the first kind and order zero. Its inclusion affects the scaling of the distribution. The von Mises distribution has properties similar to that of the Gaussian distribution. When $\theta$ equals $\mu$, probability density is maximal. The $\kappa$ is called concentration parameter because of its effect on $f(\theta)$. As the $\kappa$ approaches zero, the exponential in Function 5.2 reaches unity and so does $I_{0}(\kappa)$. At this value, the function $f(\theta)$ equals $\frac{1}{2 \pi}$ indicating a uniform distribution of probability density at smaller values of $\kappa$. At higher value of $\kappa$, the distribution becomes concentrated around $\mu$ as shown in Figure 5.4. Due to these effects, $\kappa$ is called the concentration parameter.

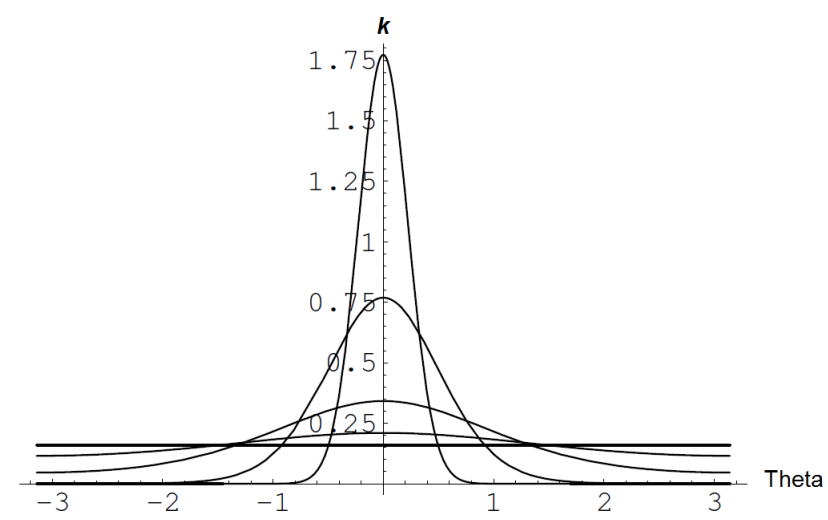

Figure 5.4 The von Mises distribution with $\kappa$ values on $y$-axis and $\theta$ values on $x$-axis (adopted from (Dhillon and Sra, 2003))

As per Dhillon and Sra (2003), to calculate probability density using a von Mises distribution there is a need to have an approximation of the concentration parameter $\kappa$. The estimation function is provided by Forbes et al. (2011) based on circular variance $\left(\sigma^{2}\right)$, concentration parameter $\kappa$ and modified Bessel function of first and zero order i.e., $I_{1}(\kappa), I_{0}(\kappa)$ respectively. The estimation function is,

$$
\sigma^{2}=1-\frac{I_{1}(\kappa)}{I_{0}(\kappa)}
$$


The von Mises distribution can be used to model uncertainty in directional information in our hints. The circular variance $(\sigma)$ can be understood as the directional uncertainty and upon specifying its value, it is possible to estimate the $\kappa$ value using the above Equation 5.4. The probability densities can thus be calculated using Function 5.2.

\subsection{Related work}

Considerable research has been done in the field of uncertainty in the geographic information domain. Fisher (1999) has presented different sources of uncertainty when working with well-defined and poorly defined objects and how they can be modeled using a probabilistic approach or fuzzy set theory. He even argued that any spatial analysis without considering the presence of uncertainty in the data is of limited use and can affect the value of the analysis results. Fisher's argument was later demonstrated by Rowe (2005) who got misleading results while studying the impact of species distribution when uncertainties associated with localities were not considered. Hunter (1999) provided a general strategy to manage uncertainty when working with geographic information. Hunter and Goodchild (1996) further provided the methodology for reporting uncertainty for spatial databases and communicating uncertainty to users using new visual methods instead of hard-copy maps with cartographic symbols (Hunter and Goodchild, 1996).

The research on uncertainty also addresses the representation and reasoning with uncertain spatial objects qualitatively, with an objective to translate them to other data types and apply on them operators that can be used in database or GIS systems. Often the research is dedicated to uncertain regions. Indeterminate boundary regions (Beaubouef et al., 2007; Bittner and Stell, 2000; Clementini and di Felice, 2001; Cohn and Gotts, 1996; Roy and Stell, 2001) describe an uncertain region as a pair of crisp regions, one enclosing the other. The inner region gives the certain part while the outer region is the broad boundary which delineates limits of the range of uncertainty. Reasoning with topological relations using region-connection calculus is discussed in (Bittner and Stell, 2000; Cohn and Gotts, 1996; Randell et al., 1992; Roy and Stell, 2001). Distance-based reasoning when working with positional information using cardinal directions is discussed in (Frank, 1996; Goyal and Egenhofer, 2000; Ligozat, 1998), whereas direction-based reasoning based on the orientation of the reference object is discussed in (Freksa, 1992; Moratz et al., 2003, 2005; Wallgrün et al., 2007; Zimmermann and Freksa, 1996).

Spatial relations play an important role when describing an object in natural-language. Any spatial relation may give different meaning when used in connection with different types of object. Mark and Egenhofer (1994), found that spatial relations, such as 'enter', 'cross', 'across', 'through' used by humans to describe objects in natural language make use of all details of the 9-intersection model. Their study was extended by Shariff et al. (1998) by using 59 terms used as spatial relations in natu- 
ral language to bridge the gap between them and computational models and their work became the basis of exploiting natural language in query processing. Their work was later extended by Xu (2007), Xu and Mark (2007) in formalizing the natural-language relations used in connections between linear objects with topological and metric properties.

In (Kiiveri, 1997), an analytical error model for positional uncertainty is proposed which can be applied to both vector or raster datasets. In identifying positional uncertainty, Ni et al. (2003) propose probabilistic models and perform spatial queries working on errors arising from a data acquisition process. Every vertex of a polygon is associated with a normal probability distribution giving the positional error for the vertex, and the uncertain polygon is given by possible realizations constructed from vertex locations at a certain probability level. Their work focuses on efficient ways of answering spatial queries, e.g., probabilistic range query (i.e., vertices of an uncertain polygon within a given region) above a certain probability level, and probabilistic distance.

Some works also address modeling the uncertainty of a place location that has been found from information extracted from the web (Jones et al., 2008; Ma et al., 2008). The main problem tackled in (Jones et al., 2008 ) is the representation of regions described by vernacular names, where neither have an official name nor a crisply defined spatial extent. The names of such vague places are often used while doing a search on the web. The paper proposes a method to model such vague places using knowledge harvested from the web. Names of vague places are also found in many web documents, frequently accompanied in text by the names of more precise co-located places that lie within the extent of the target vague place. The authors explain how to construct a density surface to represent a vague place based on the frequencies of co-occurrence of named places.

The authors of (Wieczorek et al., 2004), (Guo et al., 2008) and (Liu et al., 2009) model locations and their uncertainty from textual descriptions of locality in natural history collections. In (Wieczorek et al., 2004) they propose a point-radius method to model location and associated uncertainty, driven by an objective of efficiency and simplicity for storing and maintaining uncertain locations. An uncertain location is represented by a circle, with a radius that is calculated as the sum of errors from the different sources of uncertainty. They compile a list of most common types of textual description, consequently derive the sources of uncertainty in these descriptions, and via examples, provide ways to estimate the maximum error (uncertainty) for each source. The point, i.e., centre of the circle, is the target location and is given or easily calculated from the text description.

Guo et al. (2008), first proposed to develop models of uncertainty based on probability distribution. For a single case of 'distance in a specific direction', he demonstrated how various factors contributing to uncertainty w.r.t. the reference point and the target locality can be modeled. Further, Liu et al. (2009) proposed various probability operations that can be applied on probability rasters to further refine the location of a target 
locality. If the uncertainty fields of the target locality are derived from two independent sources, then the product of those two fields can be used to refine and represent its probability distribution. Ma et al. (2008), provides a probability distribution approach, to model uncertain event locations in application that requires situational awareness. They used normal distribution and similar refinement operation as proposed by Liu et al. (2009) to combine multiple expressions from textual descriptions.

Our approach, described below, applies an analytical approach for computing uncertainties from the spatial hints. We define a probability density function for each type and overlay rasters created using those functions for integrating hints of a gazetteer entry to derive the area of certainty where the target locality is expected to be.

\subsection{Uncertainty in spatial hints}

We identified different types of spatial hints present in our gazetteer in Section 2.4. These spatial hints contain information that may affect the geocoding process. There are various factors that can influence uncertainty and are inherent in locality descriptions (Wieczorek et al., 2004). The most commonly occurring information in hints is given in terms of distance and direction in reference to a line or point(s). For these, we consider two factors of uncertainty, namely uncertainty in distance measurements and uncertainty in direction measurements. These are explained in the following sections. There are other hints some of which describe a locality between a point and a line feature or at a certain elevation or between-two-points but do not carry direction or distance information. Depending on the hint type, the uncertainties need to be defined. In this research project, we study uncertainties in three hint types that occur most frequently in our corpus and are described in Section 5.6.

\subsubsection{Uncertainty due to distance measurement}

While taking any measurement the accuracy of distances or the calibration of the measurement instrument are hardly ever recorded. Hence, finding the uncertainty in distance measurement is difficult. To overcome this problem, Wieczorek et al. (2004) suggested two methods. In the first method, when the distance is mentioned as non-integer number e.g., $5.75 \mathrm{~km}$, it should be converted to a mixed number with simplest fraction i.e., $5 \frac{3}{4}$. The uncertainty should be then calculated as $1 /$ denominator of the fraction part. Thus, for distance of $5.75 \mathrm{~km}$, uncertainty would be one divided by the denominator i.e., 4 resulting in $0.25 \mathrm{~km}$ of uncertainty. In the second method, when distances are mentioned as integer in powers of 10 , e.g., $120 \mathrm{~km}$ or $100 \mathrm{~km}$, then uncertainty is postulated to be $0.5 \times 10^{x}$, where $x$ is the number zeroes are used. For example, if the distance is $150 \mathrm{~km}$, then $x=1$; which gives uncertainty $=$ 
$0.5 \times 10^{1}$, so uncertainty for $150 \mathrm{~km}$ is $5 \mathrm{~km}$. Similarly when the distance is $200 \mathrm{~km}$ then the uncertainty is $0.5 \times 10^{2}=50 \mathrm{~km}$.

In our gazetteer descriptions, the distances are mentioned as integer numbers and they do not always come as integers in powers of ten. Hence to estimate the uncertainty reported in our hints, we adopted a different approach. We believe that when a person reports a distance like $57 \mathrm{~km}$ or $63 \mathrm{~km}$ it is the unit value of distance that carries most uncertainty and that the distance in tens value comes with a higher level of confidence. Thus, as a rule the maximum uncertainty that we associate with a given distance is equal to the unit distance measurement. The two exceptions to this rule are distances in perfect tens and distances above 100. The maximum uncertainty that we associate with these distances is $10 \mathrm{~km}$. These rules of uncertainty will be used to create probability rasters for spatial hints. In the following section, we describe our approach for creating probability rasters.

\subsubsection{Uncertainty due to direction measurement}

Directions from a reference point are oftentimes expressed using compass directions, for example, '131 km N of Lorena'. It is difficult however, to know whether the authors notion of north is true north or some deviation from it. This leads to uncertainty in directional information and it remains to identify how much deviation around the given direction should be considered as uncertainty. To calculate directional uncertainty from compass directions, Wieczorek et al. (2004) suggested to use $45^{\circ}$ of angular uncertainty (each side of main direction) for cardinal directions i.e., E, $\mathrm{W}, \mathrm{S}$ and $\mathrm{N}$, and $22.5^{\circ}$ of angular uncertainty (each side of the main direction) for ordinal directions, i.e., NE, SE, NW, SW and $11.25^{\circ}$ (each side of main direction) for inter-ordinal directions, such as ENE, NNE, ESE, SSE and others.

We believe that this approach gives too wide angles of uncertainty and that for cardinal directions. For example, uncertainty angle for cardinal directions, say North, covers half of the compass rose between East and West. Also, in this approach cardinal and ordinal directions have been grouped separately with different uncertainties. In our corpus, the authors have used cardinal and ordinal directional terms as a part of the same linguistic group and that has also been our motivation to categories them in one group of 'full directions' in Section 4.3.2. From the same point of view we believe that uncertainties should also be similar for cardinal and ordinal directions. Further, we believe that since the author uses $\mathrm{N}$ and $\mathrm{NE}$ (say), his notion of $\mathrm{N}$ must be more restricted that does not go beyond NE and NW. We have hence considered smaller angles of uncertainty for cardinal direction. For both cardinal and ordinal directions we consider a maximum angular uncertainty of $45^{\circ}$ (i.e., $22.5^{\circ}$ on each side of the main direction). For inter-ordinal directions such as ENE, NNE, we consider even smaller angles of uncertainties since we believe that the use of these very specific directions must be in a more restricted sense. Hence, a maximum angular uncertainty of 
$22.5^{\circ}\left(11.25^{\circ}\right.$ on each side of the main direction) was adopted for interordinal directions. These uncertainties will be used to create rasters in Section 5.6.1.

\subsection{General approach to probability raster creation}

In this section, we discuss the general approach to raster creation in which we create probability rasters for different hint types. This approach is customised for each hint type, depending on the information available in the hints.

\subsubsection{Creation of raster extent}

For each type of spatial hint, there are reference geometries with respect to which the target locality is described at a particular distance and/or in some direction. As a first step, we confirm that the reference geometry is represented in WGS84. The other components of the hint text, like distance, direction are used to determine the extents of the raster. The distance and/or direction calculations are carried out in a metric projection system (UTM) based on which the extent of the raster is determined. The raster extent is then converted to WGS84 representation to determine its width and height. In this projection system, we then identify the upper left corner of the raster extent as its origin. Within the specified extents, an empty raster is created with a specification that all cells are of equal size and square shape. We have also fixed the raster cell size to 7.5" $\times$ 7.5." Thus, we can identify here some organizational principles that are required for creating raster extents. These are:

- all reference geometries are provided in WGS84 representation,

- the extents of the probability raster are fully determined by the choice of a number of parameters, like distance (in meters), direction (in radians) and they depend on the specific hint type,

- distances and directions are provided in meters and radians respectively,

- all distance and direction calculations are carried out in a local metric projection system and are then converted to WGS84 representation,

- all cells must be square and equal in size (7.5" $\times 7.5$ "),

- the raster grid should be fixed at 0.0 latitude and longitude.

The next step of assigning the cell value to each cell in the raster, is elaborated in the following section.

\subsubsection{Assigning cell values}

The cell value in a raster represents the probability of the target locality being inside that cell. As discussed in Section 5.2, we identified probability density functions assign probability over the raster space. For hints 
containing distance components, a probability density function (pdf) based on a Gaussian distribution is used, and for direction components a von Mises distribution is used. Depending on the hint type, its pdf is customized. These distribution functions determine the probability density at any point within a raster. In the implementation of our raster creation functions, we use the function to determine pdf values for the cell's corners and its midpoint. Each point's probability density is interpreted as an elevation, and we determine the accumulated probability per raster cell as a volume under the so constructed 5-points polyhedral surface, which can be seen as a tiny TIN over the cell space. We illustrate these computations in Figure 5.5, in which $P, Q, R, S$ are the corner points of a raster cell and $p, q, r, s$ are the points representing probability density for $P, Q, R, S$ respectively. By $C$ we denote the midpoint of the raster cell, and ' $c$ ' is its probability density value. On joining the five-points $p, q, r, s$ and $c$, a solid object is formed and the shape of this solid object is an enneahedron, i.e., a nine-sided volume object with the raster cell as its base.
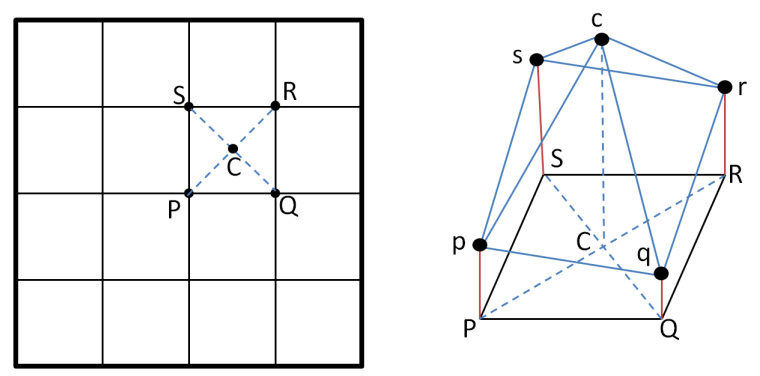

Figure 5.5 Illustration of the five points of a raster cell used in determining the cell probability value

The probability value assigned to the cell is the total volume of this enneahedron and to calculate it, the volumes under each of the four triangles (cpq, $c s p, c q r$ and $c s r$ ) are summed up. We call this process the five-point triangulation approach. Observe that we stated that $\mathrm{C}$ is the midpoint of the raster cell; our default choice for it is that is the cell centroid. But occasionally, we can opt for a better choice, as long as an enneahedron results. Special cases arise when reference geometries (especially points or linestrings) intersect the raster cell, and affect the accumulated probability by their presence. We return to this issue in Section 5.6.2. After the cell values are determined for all cells in the raster, the complete raster is normalized by summing up the probability values of all cells, and dividing every cell value by this sum, to ensure that the new summation equals 1.0. This ensures that our raster probability computations do indeed result in a true probability assignation. In the following section, we explain how the rasters are created for each specific hint type. 


\subsection{Raster creation for spatial hints}

The probability rasters explained above quantify the probabilities at points locations. For different hint types, the general approach of raster creation is the same, but depending on input parameters related to distance and direction, the raster extent calculations are customised. Similarly, the pdf used for probability calculation also needs to be customised depending on the hint type. In the following sections, we elaborate on how the process of raster creation varies per hint type.

\subsubsection{Distance-direction from a reference point}

In this section, we describe probability raster creation for a hint type in which a target locality is described to be at a particular distance and in a particular direction from a reference point. For example, in '25 km SE of Jundiaí', we create a probability raster for a point that is estimated to be ' $25 \mathrm{~km}$ ' 'SE' of 'Jundiaí'.

As discussed in Section 5.5, the extent of a raster depends on the parameters that are textually extracted from the hint. In this hint, we have three known components: a reference point $(r)$, distance $(d)$ and a direction from a reference point $(\theta)$ indicative of the position of a target locality $(q)$. We developed a function that determines the point geometry of the target point assuming that the provided distance, direction and reference point are exact (Figure 5.6). Next, with $r$ as centre, two circles ${ }^{1}$ are created namely an outer $\left(C_{1}\right)$ and inner circle $\left(C_{2}\right)$. The dimensions of the outer circle and inner circles are determined by distance $d$ and distance uncertainty $\sigma_{\text {dist }}$ that we choose, such that the radius of the outer circle from the reference point is equal to $d+\sigma_{\text {dist }}$ and the radius for the inner circle is $d-\sigma_{\text {dist }}$ as shown in Figure 5.6.

The geometric difference of these outer and inner circles gives us the circle donut shape. Our target locality is expected to be somewhere in this donut shape. This area still needs to be further restricted by the direction hint. We created another function that uses direction information $\theta$ and direction uncertainty $\left(\sigma_{d i r}\right)$ as its input to create a direction sector emanating from the reference point. The two sides of the direction sector represent the angles of $\theta-\sigma_{d i r}$ and $\theta+\sigma_{d i r}$ and are shown in Figure 5.6. This direction sector covers both sides of the main direction angle and the length of this sector is $2\left(d+\sigma_{\text {dist }}\right)$ to ensure that the sector sufficiently stretches beyond the donut. The intersection of this sector with the circle donut gives us the area where we expect the target locality to fall. The minimum bounding rectangle that envelopes this intersected area forms the extent of the raster (Figure 5.6).

It is worthwhile to mention how the distance and direction uncertainty values are chosen. As discussed in Section 5.4.1, for a distance of $25 \mathrm{~km}$ (say) we associate a maximum uncertainty equal to the unit distance, i.e.,

\footnotetext{
${ }^{1}$ In essence by 'circle' we mean a disc, namely a 2-dimensional feature rather than a linestring. However, for reasons of simplicity we use the word 'circle' in this chapter.
} 


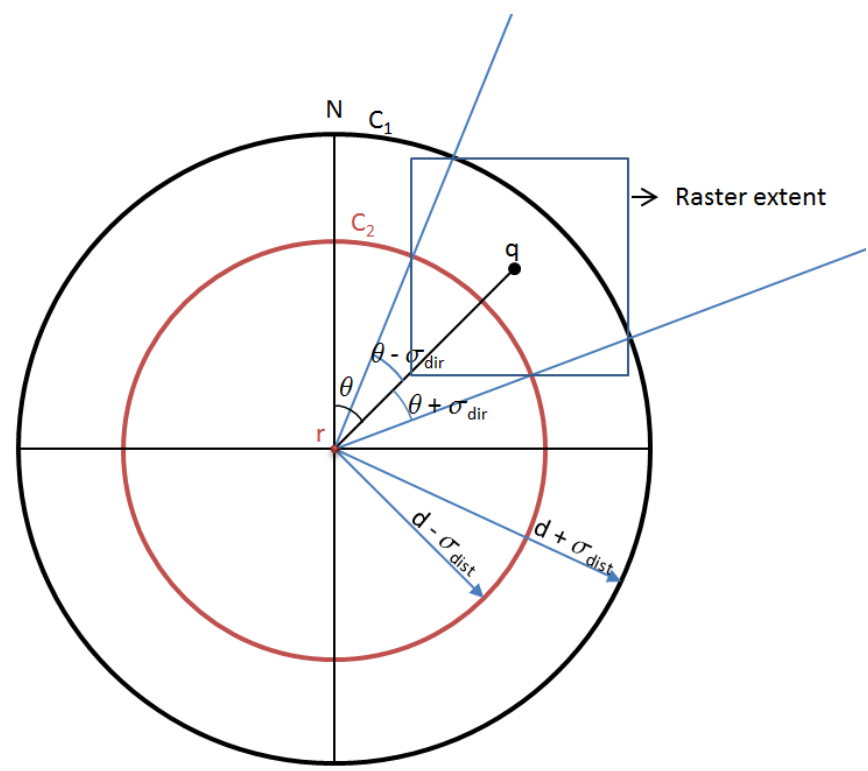

Figure 5.6 Creation of raster extents for hint type distance-direction from a reference point

$5 \mathrm{~km}$ for this hint. Within the $5 \mathrm{~km}$ distance, we use the 68/95/99.7\% rule of standard deviation (Moore et al., 2012) and create three points of distance uncertainties $\left(\sigma_{\text {dist }}\right)$. Similarly, as discussed in Section 5.4.2, for a cardinal or ordinal direction (say), the maximum direction uncertainty is considered to be $22.5^{\circ}$ on each side of the direction angle. Here too, we used a 68/95/99.7\% rule of standard deviation and created three points of direction uncertainties $\left(\sigma_{d i r}\right)$ for raster creation. For entries that have both distance and direction hint, e.g., $25 \mathrm{~km} \mathrm{SE}$ of a reference point, we created nine rasters using the combinations of three $\sigma_{\text {dist }}$ and three $\sigma_{d i r}$ values. While Wieczorek et al. (2004) used a single distance and direction value to estimate the uncertainty associated with a hint, we used nine combinations of distance and direction uncertainties because we do not know which combination of $\left(\sigma_{\text {dist }}, \sigma_{\text {dir }}\right)$ is the best performing parameter for raster creation. In Section 5.7, we discuss our approach to find the best performing parameter.

Once the raster extents are defined, then in line with the explanation provided in Section 5.5, the width and height of the rasters are determined. An empty raster is created and the cell values are computed.

Computing the cell values in these rasters is not a straight-forward process since we have two components: distance and direction. As mentioned in Section 5.2, the probability density of these two components can be separately expressed using a normal distribution and a von Mises distribution, for distance and direction respectively. However, for this particular hint type we need to consider both of these distributions in a combined way to calculate the probability values since they together 
describe the location of a target locality. Thus, there is a need for a probability function that combines both distribution functions. As per Liu et al. (2009), when the hint has two components that are not conflicting with each other and can be expressed using two distributions then the combined distribution can be taken as product of those two distributions. In this hint type, the distance hint is independent from the direction hint and hence a combined pdf for a point $x$ in a direction $\theta$ (from $r$ ) can be computed as the product of normal and von Mises distribution as follows:

$$
f(x, \theta)=\left(\frac{e^{\kappa \cos \left(\theta-\mu_{\text {dir }}\right)}}{2 \pi I_{0}(\kappa)}\right)\left(\frac{1}{\sigma_{\text {dist }} \sqrt{(2 \pi)}} e^{\frac{-\left(m-\mu_{\text {dist }}\right)^{2}}{2 \sigma_{\text {dist }}}}\right),
$$

where $m$ is distance of $x$ from $r, \mu_{d i r}$ is the mean direction in radians, $\mu_{\text {dist }}$ is mean distance, $\sigma_{\text {dist }}$ is the distance uncertainty, $\kappa$ is the concentration parameter calculated from $\sigma_{d i r}^{2}=\left(1-\frac{I_{1}(\kappa)}{I_{0}(\kappa)}\right)$ (Equation 5.4). Here, $\sigma_{d i r}$ is the direction uncertainty, $I_{0}(\kappa)$ and $I_{1}(\kappa)$ are modified Besseli functions of zero and first order respectively and are used for scaling and probability distribution. We consider the value of $\mu_{\text {dist }}$ as 0.0 .

Figure 5.7 Illustration of a raster for the case of ' $25 \mathrm{~km} \mathrm{SE}$ of Jundiaí'

Probability density function of Equation 5.5 is used to calculate the probability values for each of the five-points in a raster cell. The cell values over the whole raster are then computed using the five-point triangulation approach detailed in Section 5.5.2. In the end, the raster is normalised. Figure 5.7 shows a raster that was created for the hint ' $25 \mathrm{~km}$ SE of Jundiaí'. The inner red portion indicates the highest probability and within it lies a cell with maximum probability density. As we go outwards from this red portion the probability reduces continuously. 
The light pink area of the raster is an area of very low probability values. Any location outside of the raster is associated with probability 0 for this hint.

For this research project, we made the rasters for 108 such hints containing the combination of distance and direction information. Our sample for all hint types was carefully picked to ensure that we have a sufficient number of entries containing more than one hint of the same or different type. This was necessary for the overlay of rasters in Section 5.8 .

\subsubsection{Distance along/from some linear feature}

In this section, we describe probability raster creation for a hint type in which a target point is described to be located from a reference point at a distance along a line feature. For example, 'on north bank of Rio Amazonas [0010/4900 (USBGN)], $90 \mathrm{~km}$ downstream from Santarém [0226/5442 (USBGN) ]'. In this hint, the reference point is the city of 'Santarém' on the banks of 'Rio Amazonas' (which is considered a line feature) and our target locality is ' $90 \mathrm{~km}$ ' away from the reference point in downstream direction of the river (Figure 5.8).

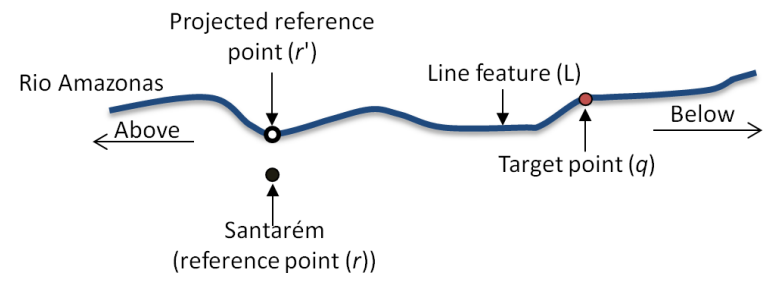

Figure 5.8 Illustration of along the line example showing position of reference line $(L)$, reference point $(r)$, its projection $\left(r^{\prime}\right)$ on line and target point $(q)$

To define the raster extents, we first verify our input geometries. Since the reference point can only be located on the banks of the river, we take the projection of the reference point $(r)$ on a line feature to create a projected reference point $\left(r^{\prime}\right)$ on line feature.

As a next step, we verify whether the available length of the line feature is more than the stated distance (upstream or downstream) or not. The raster will only be created if there is sufficient length. For every stated distance $d$, like $90 \mathrm{~km}$ in the example hint, there is a distance uncertainty $\sigma_{\text {dist_along }}$ which is used to identify that section of the line feature where the target locality may be located. This selected section of the line feature is located between two points, plotted from $r^{\prime}$ and the computation of those two points is given below,

$$
\begin{aligned}
& P_{1}=d+\sigma_{\text {dist_along }}, \\
& P_{2}=d-\sigma_{\text {dist_along. }} .
\end{aligned}
$$


The selected line feature (totally spatially embedded within $L$ ) between these points can be called $P_{1} P_{2}$. The plotting of points and identification of the line section is demonstrated in Figure 5.9. In some cases, it is possible that the length of the line feature falls short for plotting $P_{2}$. In such cases, we extend the line, upto the $P_{2}$. Once the $P_{1} P_{2}$ has been identified, an envelope $\left(E_{1}\right)$ is created around it.

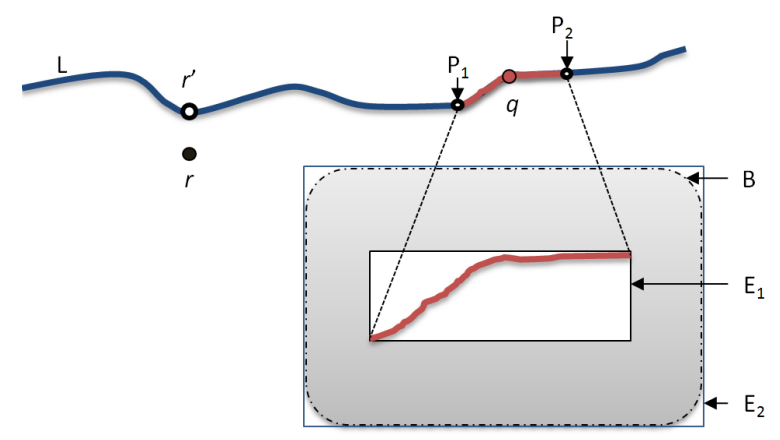

Figure 5.9 Creation of raster extents for hint type along/from the line feature

Since the target locality can only be beyond the bank of the river and we do not know how far beyond, thus, we consider another distance uncertainty on each side of the line feature, within which the target locality may be located. We call this distance uncertainty $\sigma_{\text {dist_from }}$ and a buffer $(B)$ is created at a distance of $\sigma_{\text {dist }}$ from around the envelope $\left(E_{1}\right)$. Once the buffer has been formed around the selected line feature, an envelope $\left(E_{2}\right)$ is created around the buffer $(B)$ (Figure 5.9). This envelope $\left(E_{2}\right)$ defines the extent of the raster. The width and the height of the raster are then determined and an empty raster is created. For this hint type, we need to specify two distance uncertainties: $\sigma_{\text {dist_along }}$ and $\sigma_{\text {dist_from }}$. To specify $\sigma_{\text {dist_along }}$ we assume the maximum distance uncertainty equal to the unit distance in the given distance as explained in Section 5.4.1. Within that maximum distance uncertainty, three points of uncertainty are specified using the $68-95-99.7 \%$ rule of standard deviation as mentioned in Section 5.6.1. To specify $\sigma_{\text {dist_from }}$, we assume a maximum distance uncertainty of $10 \mathrm{~km}$ (each side of the line feature) since this distance is not given in the hint and we consider the fact that rivers in Brazil can be as wide as $15 \mathrm{kms}$ like the Rio Amazonas. Within this maximum distance uncertainty of $10 \mathrm{~km}$, three points of uncertainty were specified using the 68-95-99.7\% rule of standard deviation. Using the combinations of three $\sigma_{\text {dist_along }}$ and three $\sigma_{\text {dist_from }}$, nine rasters are created for each hint of this type.

To distribute the probabilities within an empty raster we need to consider two pdfs-one for distributing the probabilities along the line and the other for distributing the probability away from the line. For both we use a normal probability distribution function as we expect probability densities to be highest at point $q$ and reduce continuously as we move away from it. Thus, the combined pdf for calculating probability 
density at point $x$ in a raster is given as follows,

$$
\begin{aligned}
f(x)= & \left(\frac{1}{\sigma_{\text {dist_along } \sqrt{(2 \pi)}}} e^{\frac{-\left(m-\mu_{\text {dist_along })^{2}}\right.}{2 \sigma_{\text {dist_along }}}}\right) \\
& \left(\frac{1}{\sigma_{\text {dist_from } \sqrt{(2 \pi)}}} e^{\frac{-\left(n-\mu_{\text {dist_from })^{2}}\right.}{2 \sigma_{\text {dist_from }}^{2}}}\right),
\end{aligned}
$$

where $m$ is distance of $x$ from $r^{\prime}, n$ is distance of $x$ from $q, \mu_{\text {dist_along }}$ is mean distance along the line feature and $\mu_{\text {dist_from }}$ is the mean distance away from the line feature. We consider the value of both $\mu$ as 0.0 .

Figure 5.10 Illustration of a raster for the case 'on north bank of Rio Amazonas [0010/4900 (USBGN)], 90 km downstream from Santarém [0226/5442 (USBGN) ]'

The cell value is then computed using the five-point triangulation approach detailed in Section 5.5. Under that approach the probabilities at the four corners of the cell and the cell centroid are used for cell value computations. In this hint type, there will also be those cells through which the line passes. For those cells the fifth point will not be the cell centroid, but the midpoint of the line segment that passes through that cell. The raster can be normalised after the cell values have been computed in it. Figure 5.10 shows an example raster that was created for hint 'on north bank of Rio Amazonas [0010/4900 (USBGN)], $90 \mathrm{~km}$ downstream from Santarém [0226/5442 (USBGN)]'. The highest probability values are along the line. For this research project, we made nine rasters each for 36 such hints. From these nine rasters, we will choose a best performing raster, the process for which is discussed in Section 5.7. 


\subsubsection{Between-two-points}

In this section, we describe probability raster creation for a hint type in which a target locality $q$ is mentioned between-two-reference points $r_{1}$ and $r_{2}$ as shown in Figure 5.11. For example, in 'between Laje [1310/3925 (USBGN)] and Nazaré [1302/3900 (USBGN)]', the target locality is described to be located between the two towns of 'Laje' and 'Nazaré'.

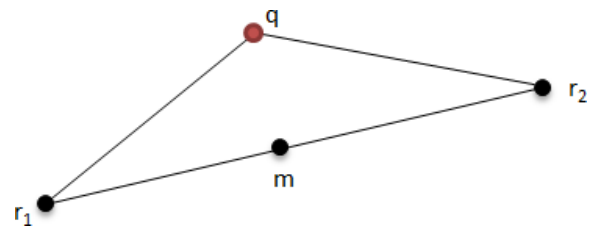

Figure 5.11 Illustration of a between-two-points example $\left(r_{1}\right.$ and $r_{2}$ are two reference points, $q$ is the target point and $m$ is the midpoint of line $r_{1} r_{2}$ )

For defining the raster extents, we need to define parameters such as distance or direction. However, in this hint type, we have two reference points $r_{1}$ and $r_{2}$ only and no distance or direction component. As shown in Figure 5.12, the distance between the two points is represented as $\left|r_{1} r_{2}\right|$ and a midpoint $m$, on that line is indicated. From this midpoint $m$ we construct a circle with radius $h$ equal to the distance between the two points, i.e., $\left|r_{1} r_{2}\right|$ (Figure 5.12). This circle gives the bounded area of probability distribution. The circumscribing envelope to this circle forms the extent of the raster. The shape of this envelope is always a square such that each side is twice the distance between the two reference points.

Once the raster extent is defined, its width and height are determined as discussed in Section 5.5, an empty raster is created before the cell values are computed.

In this type of hint, only the two reference points are provided and the target point $q$ may be located either on the line $r_{1} r_{2}$ or away from it. To represent the probability distribution for this hint type, we need two distributions, one that expresses probability distribution along the line $r_{1} r_{2}$, with highest probability around its midpoint and another distribution around the same midpoint but away from the line in perpendicular direction. Thus, the midpoint on the line between two reference points becomes the center of our raster.

To model the probability density distribution along the line $r_{1} r_{2}$, we use a normal distribution function as we expect the probability density for this hint to be highest at a mean point, i.e., the midpoint $m$ and the probability density will reduce continuously as we go away from the point $m$, towards either $r_{1}$ or $r_{2}$. Thus the pdf at any point $q$ is:

$$
f(q)=\frac{1}{\sigma \sqrt{2 \pi}} e^{\frac{-\left(\left|r_{1} q\right|-\mu\right)^{2}}{2 \sigma^{2}}},
$$




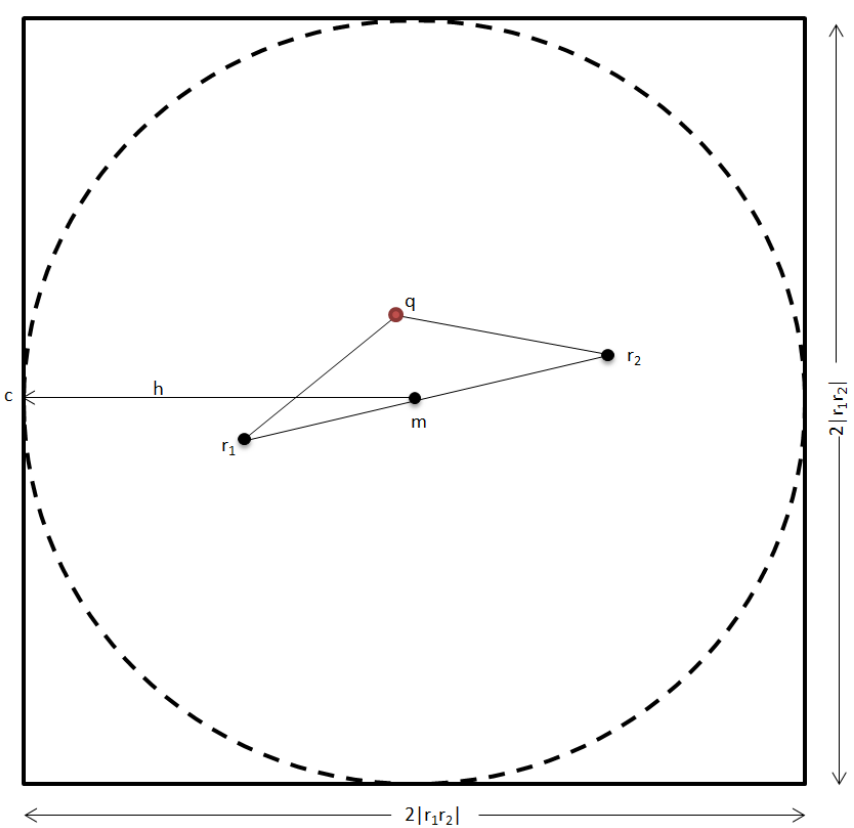

Figure 5.12 Illustration for the raster extent in the between-two-points case

where $\left|r_{1} q\right|$ is the distance between $q$ and $r_{1}$ and $\left|r_{2} q\right|$ is the distance between $q$ and $r_{2}, \mu=\frac{\left|r_{1} q\right|+\left|r_{2} q\right|}{2}$ and $\sigma=\frac{\left|r_{1} q\right|+\left|r_{2} q\right|}{2 z}$. The $\sigma$ represents a distance deviation that is affected by the chosen value of $z$. The $z$ value affects the speed at which probability density reduces as the point goes away from the midpoint, along the line.

When $q$ falls anywhere on $r_{1} r_{2}$, the $\mu$ and $\sigma$ calculations can be simplified as $\mu=\frac{\left|r_{1} r_{2}\right|}{2}$ and $\sigma=\frac{\left|r_{1} r_{2}\right|}{2 z}$. The equation shall return the maximum probability when $q$ is at $m$, i.e., the point lies in the center or midpoint of $r_{1} r_{2}$.

We still need to identify an appropriate function to model probability distribution away from $r_{1} r_{2}$. It should be such that the probability is highest when the point is on the line $r_{1} r_{2}$ and that it monotonically reduces as we move away from the line. Thus, the following function was adopted to model this behavior:

$$
g(\mathrm{q})= \begin{cases}\left(\frac{\left|r_{1} r_{2}\right|}{\left|r_{1} q\right|+\left|r_{2} q\right|}\right)^{t} & \text { if }\left|r_{1} r_{2}\right|>\max \left(\left|r_{1} q\right|,\left|r_{2} q\right|\right) \\ 0.0 & \text { otherwise }\end{cases}
$$

In the above equation, $t$ determines the rate at which probability diminishes away from the line. Using the above function, when $q$ is on $r_{1} r_{2}$, then independent of the value for $t$, the function $g(q)$ equals 1.0 . The function also carries the condition that, both $\left|r_{1} q\right|$ and $\left|r_{2} q\right|$ should be less than $\left|r_{1} r_{2}\right|$ or the probability equals zero. This implies that when the target point is farther than the distance between the two 
reference points, we consider it to fall beyond the bounded area of probability.

Finally, to model the probability density distribution for the complete raster, we apply the product of probability density function $f(q)$ and function $g(q)$, as defined in Equation 5.9 and 5.10. Thus, we use the following compound function to compute the probability density at any point $q$ in our raster.

$$
f\left(q_{b t w}\right)=\left(\frac{1}{\sigma \sqrt{2 \pi}} e^{\frac{-\left(\left|r_{1} q\right|-\mu\right)^{2}}{2 \sigma^{2}}}\right)\left(\frac{\left|r_{1} r_{2}\right|}{\left|r_{1} q\right|+\left|r_{2} q\right|}\right)^{t}
$$

In the above function, the $z$ value (used in computing $\sigma$ ) affects the distribution of probability along the line and $t$ affects the distribution of probability away from the line. Hence, to use the above function we want to use optimal values for $z$ and $t$. Unlike other hints in Section 5.6.1 and 5.6.2, we do not have given distance or direction component to estimate the uncertainty. Hence, we depend on $z$ and $t$ values for creation of rasters. Since there is infinite number of $(z, t)$ combinations, it is

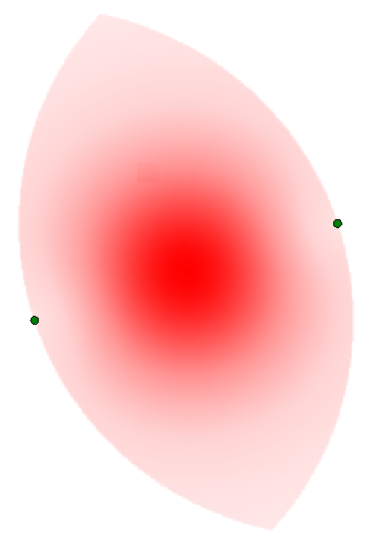

Figure 5.13 Illustration of a raster for the case 'between Laje [1310/3925 (USBGN)] and Nazaré [1302/3900 (USBGN)]' with $z=2.0$ and $t=2.5$

practically impossible to create a raster for each combination and find the best combination by comparing the rasters. Thus, it becomes necessary to find the optimal $z$ and $t$ values first before the raster creation. This process of optimization of $z$ and $t$ is discussed in Section 5.7.3. Once the probabilities are calculated for all points in raster using the optimised $z$ and $t$ values, probability densities can be calculated using the pdf of Equation 5.11. The cell values of a raster can be computed using the five-point triangulation approach detailed in Section 5.5. In the end, the raster can be normalised. As an example, Figure 5.13 shows a raster for one of the entries with assumed values of $z=2.0$ and $t=2.5$. 


\subsection{Optimisation of input parameters}

The basic purpose of creating probability rasters for each hint is to allow the overlay of rasters of different hints for the same entry and identify common areas of certainty. Thus, we need one raster per hint. In the previous section, we explained how multiple rasters can be created for a hint using different parameter values and a choice must be made from these resulting rasters for overlay analysis. To make that choice, we need to find parameter values under which the raster optimally forecasts the location of the described target location. Clearly, this must be done under a single fixed choice of parameter values for all rasters created for the hint type at hand. In the following sections, we discuss how these values are identified for each hint type.

We define optimality in the context of the creation of probability rasters for some hint type $h t$ as follows. Let $H_{h t}$ be that set of hints for type $h t$ as extracted from our gazetteer corpus, for which the described target locality is actually known. Many, but not all, hints happen to be of this type, and this allows us instant verification of our raster creation procedures. Also, let $\bar{p}$ denote input parameters for the raster creation function. For any hint $h \in H_{h t}$, our probability raster creation procedure creates a raster rast $_{\bar{p}}(h)$, by construction with fixed cell size but variable raster size size $\left(\right.$ rast $\left._{\bar{p}}(h)\right)$, expressed as the number of raster cells. If $c$ is a cell in the raster, in which the target locality is found, then $\operatorname{rast}_{\bar{p}}(h)(c)$ denotes the value of that cell $c$.

As larger rasters spread their probability over more cells, the comparison of probabilities between cells of different rasters must be done carefully. Specifically, our formula to determine optimal input parameter choices sums up probabilities over all rasters for some hint type for different entries; for comparability reasons, this must be done in a weighted setting such that different raster sizes do not have impact over the optimization process. In brief, we will be summing up weighted probabilities, accounting for raster size, and use the expression

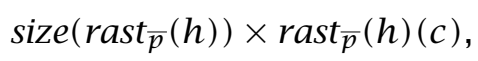

for some cell $c$. It is easily seen that the size of the raster is the weight that we must apply to make cell values of different rasters comparable.

In fact, for optimization, the cell $c$ will be the cell in which the described target location falls. For a number of hints, we happen to know that location, namely, and so we denote with target $(h)$ the raster cell in which the (described) target location for hint $h$ is known to be located.

We define a choice of values $\bar{v}$ for the parameters $\bar{p}$ for hint type $h t$ to be optimal if and only if the sum of all weighted probabilities $(w)$ is highest amongst all possible choices for $\bar{v}$. The equation for $w$ can be given as follows:

$$
\left.w=\sum_{h \in H_{h t}} \operatorname{size} \operatorname{rast}_{\bar{v}}(h)\right) \times \operatorname{rast}_{\bar{v}}(h)(\operatorname{target}(h)),
$$




\subsubsection{Optimal parameters for distance-direction from the reference point}

For this hint type, we generated probability rasters using the function discussed in Section 5.6.1, with three uncertainty values for distance $\sigma_{\text {dist }}$ and direction $\sigma_{\text {dir }}$ each, to create nine rasters. Thus, our input parameters $\bar{p}$ are the nine combinations of $\sigma_{\text {dist }}$ and $\sigma_{\text {dir }}$. To do overlay analysis using those rasters we must choose one raster per hint by finding optimal value of $\bar{p}$.

Using Equation 5.13, we find the sum of weighted probabilities of all $\bar{p}$ over all hints. Finally, $\left(\sigma_{d i s t 2}, \sigma_{d i r 3}\right)$ was found to be the most optimal combination of $\bar{p}$ for raster creation for this hint type since the sum of its weighted probabilities ( $w$ ) over all hints is highest as shown in Figure 5.14

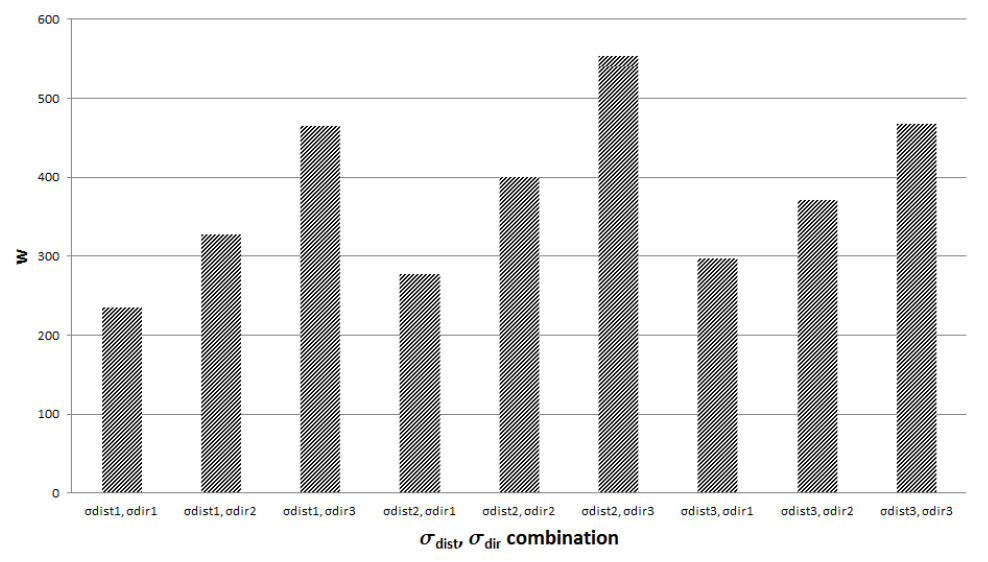

Figure 5.14 Graph of weighted probabilities vs. $\sigma_{d i s t}, \sigma_{d i r}$ combinations

Using the optimal parameters for this hint type, the rasters for overlay analysis (in Section 5.8) can be chosen.

\subsubsection{Optimal parameters for distance along/from the reference object}

As discussed in Section 5.6.2, we generated nine probability rasters for each hint, using three uncertainty values for distance along the line feature $\sigma_{\text {dist_along }}$ and distance away from the line feature $\sigma_{\text {dist_from }}$. For the overlay analysis, one of those nine rasters must be chosen by finding the optimal values of $\bar{p}$ i.e., optimal combination of $\sigma_{\text {dist_along }}$ and $\sigma_{\text {dist_from. }}$. As in the previous section, using Equation 5.13, we found the sum of weighted probabilities of all $\bar{p}$ over all hints for all nine choices of $\bar{p}$. The $\left(\sigma_{\text {dist_along2 }}, \sigma_{\text {dist_from } 3}\right)$ combination was again found to have the highest sum and was thus selected as optimal as shown in Figure 5.15. 


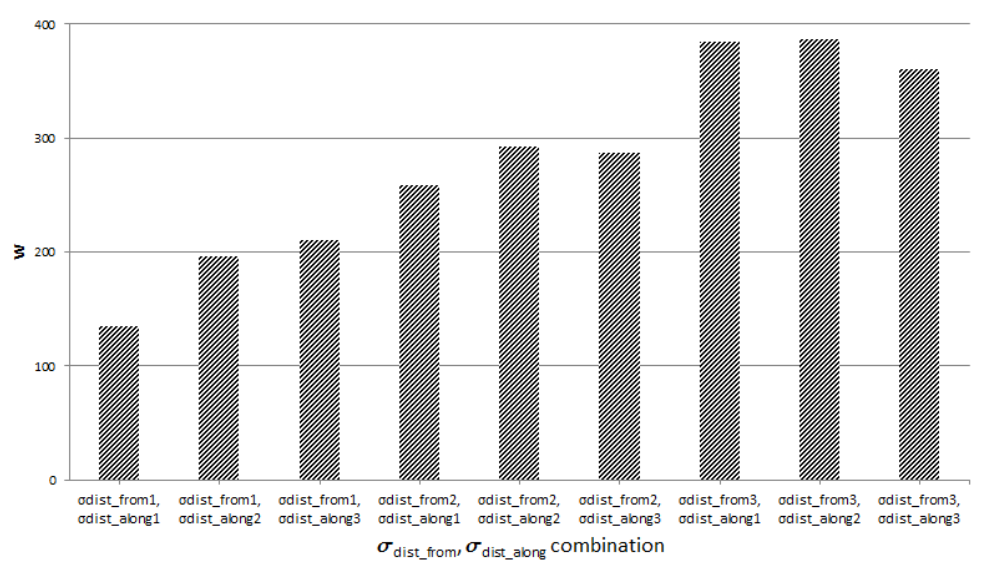

Figure 5.15 Graph of weighted probabilities vs. $\sigma_{\text {dist_along }}, \sigma_{\text {dist_from }}$ combinations

Using the optimal parameters for this hint type, the rasters for overlay analysis (in Section 5.8) can be chosen.

\subsubsection{Optimal parameters for between-two-points}

For rasters of this hint type, we use the pdf of Section 5.6.3. The input parameters are $z$ and $t$; raster creation for this type is of high time-complexity, making it practically impossible to create rasters for many different $(z, t)$ combinations. As a consequence, we developed an approximation function $\widetilde{\text { rast }}_{z, t}(h, c)$ that does not create a full raster, but computes the approximate value of a single raster cell $c$ only. This new function is of much lower time-complexity because it does not rely on computing a full raster, and as it does not perform the final normalization step.

The question then arises what is the relationship between rast $_{z, t}(h)(c)$ and $\widetilde{\text { rast }}_{z, t}(h, c)$. For the sake of simplicity, let us call these two $p(c)$ and $a(c)$, respectively, as shorthands for the precise, and approximate cell value for cell $c$. We also use $s$ as a shorthand for size $\left(\right.$ rast $\left._{\bar{p}}(h)\right)$.

We studied the correlation between $p(c)$ and $a(c)$ and the role of $z$ and $t$ in that correlation. To do so, we calculated $p(c), a(c)$ and $s$ of some 35 hints with varying $z$ and $t$ values. For each pair $(z, t)$ we determined the ratio $\frac{a(c)}{p(c)}$. Upon plotting the $s$ and this ratio, we found that a parabolic relationship exists between them which can be expressed as:

$$
s=d(z, t)\left[\frac{a(c)}{p(c)}\right]^{2},
$$

To determine the precise nature of the dependency of $d(z, t)$ on values for $z$ and $t$, we determined a range of sensible values for both 
parameters: $z \in[1.5,3.5]$ and $t \in[1.5,9.5]$. Subsequently, we found that $d$ correlates with $t$ linearly, as expressed in Equation 5.15, and that the coefficients in that equation depend on $z$ through two third-order polynomials, provided in Equations 5.16 and 5.17. For the first linear correlation, we found a correlation coefficient of 0.99 , while for the two polynomial correlations the correlation coefficient was 0.98 and 1.0 respectively.

$$
\begin{aligned}
& d(z, t)=\alpha_{z} t+\beta_{z} \\
& \alpha_{z}=\left(-0.0001 z^{3}+0.0006 z^{2}-0.0015 z+0.0015\right) \\
& \beta_{z}=\left(-0.000003 z^{3}+0.00004 z^{2}-0.0002 z+0.0003\right)
\end{aligned}
$$

Equation 5.14 can also be written as

$$
p(c)=a(c) \sqrt{\frac{d(z, t)}{s}},
$$

which allows to determine a value for $p(c)$ from $a(c), d(z, t)$ and $s$, given a choice for $z$ and $t$ values. Also note that $p(c)$ is our shorthand for rast $z, t(c)$ and that $c$ can play the role of target $(h)$ in Equation 5.13 above.

Substituting Equation 5.18 into Equation 5.13, with appropriate parameter substitution for $c$ by $\operatorname{target}(h)$ we obtain the following optimization target:

$$
\sum_{h \in H_{h t}} \operatorname{size}\left(\operatorname{rast}_{z, t}(h) \times \operatorname{a(target}(h)\right) \sqrt{\frac{d(z, t)}{\operatorname{size}_{\left(\text {rast }_{z, t}(h)\right.}}},
$$

which simplifies to

$$
\sum_{h \in H_{h t}} a(\operatorname{target}(h)) \sqrt{d(z, t) \operatorname{size}_{\left(\text {rast }_{z, t}(h)\right.}} .
$$

For different $z, t$ combinations, Equation 5.20 gives the summation of weighted probabilities. This will help in determining at which combination of $z, t$ the sum of weighted probabilities is highest. Since we optimize for known target points, our function implementation is such that when the target point $q$ is on $r_{1} r_{2}$, then independent of the value of $t, g(q)$ equals 1.0 (Section 5.6.3). When optimizing using approximation function $\widetilde{\text { rast }}_{z, t}(h, c)$, we noticed that, when the point $q$ is on or close to $r_{1} r_{2}$, i.e., when fractional part $\frac{\left|r_{1} r_{2}\right|}{\left|r_{1} q\right|+\left|r_{2} q\right|}$ in $g(q)$ is higher than 0.975 , our approximation function was not performing well since the value of $t$ for such cases was very high and beyond any sensible value. The reason is that $d(z, t)$ is an approximation that was devised with relatively low numbers for $t$, and thus it is not valid for high $t$ values. Thus, we used 


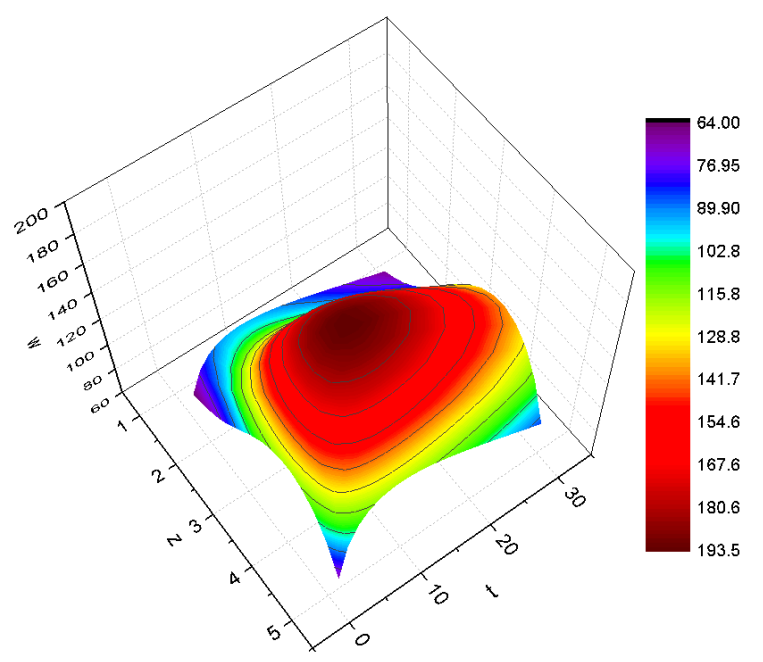

Figure 5.16 3D surface plot of showing weighted probability $(w)$ peaking at $z=4.0$ and $t=10.5$

those entries whose fractional part of $g(q)$ is 0.975 or less, to find the optimal values of $z$ and $t$. We calculated the sum of weighted probabilities for a number of $z, t$ combinations using Equation 5.20. Figure 5.16 shows a three-dimensional surface plot of $w$ peaking at $z=4$ and $t=10.5$. At finer levels of optimization, a true peak of weighted probabilities was found at $z=3.9180$ and $t=9.9505$. Using these as optimal values of $z$ and $t$ we created rasters for 35 such hints. These were then used for raster overlay and other analyses, as discussed in later sections.

\subsection{Raster overlay}

So far, we have created probability rasters for different spatial hints describing the position of the same locality as obtained from entry description. By overlaying the rasters of different hints of the same entry we cross-validate them and find the common area of certainty where the target locality is expected to fall. Thus, in this section we will overlay the rasters of hints available in an entry. The process of raster overlay was done in PostGIS environment using the built-in raster ST_MapAlgebraExpr function. The process of overlay within that function is described here.

By construction, our rasters are always overlay-compatible because they are registered in the same spatial reference system, and they have apply an aligned standard cell grid. Upon overlaying them, the area where the two rasters overlap is called the 'area of intersection' and a new 'resultant raster' is created within the dimensions of the 'area of intersection'. The resolution of the resultant raster is the same as the raster that is used as the first argument in overlaying. Since the resolution 
of all our rasters is identical, the resolution of the resultant raster is also the same as both overlaying rasters. In ST_MapAlgebraExpr function, the probability values of cells in the resultant raster can be calculated by either adding the probabilities of overlapping rasters, or taking their average or taking their product. We chose to compute the probabilities of resultant rasters by multiplying the individual probability values of overlapping rasters in view of the 'multiplication rule for independent events' from probability theory (Moore et al., 2012). The rule states that when the occurrence of two events $A$ and $B$ is independent of each other and the two events overlap (Figure 5.17), then their combined probability in the overlapping area can be calculated by multiplying their individual probabilities. This operation of computing the combined probability can be expressed as follows,

$$
P(A \cap B)=P(A) \times P(B) .
$$

where, $P(A)$ is the probability of event $A, P(B)$ is the probability of event $B$ and $P(A \cap B)$ is the combined probability of cell common to both $A$ and $B$.

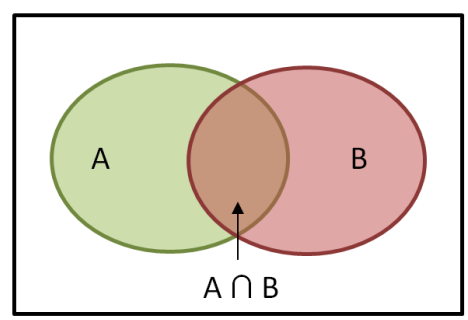

Figure 5.17 Intersection of two events $A$ and $B$

In our entries, various hints describe the same locality but each of those hints is independent of others, i.e., the occurrence of one, does not change the probability of occurrence of the other hint(s). Thus, this rule can be applied to our rasters' overlay. Since the total probability of the individual rasters is 1 , the total probability of the resultant raster also does not exceed 1 . This process of carrying out an operation between two sets, each having values in the range of 0 and 1 , and arriving at a resultant set of values, also ranging between 0 and 1 is also called Triangular norm or T-norm (Klement et al., 2004) and expressed as $T:[0,1] \times[0,1] \rightarrow[0,1]$. Multiplication of the two such sets is called 'Product T-norm' and can be expressed as follows: $T(a, b)=a b$.

The raster overlay process can be carried out using only two rasters at a time in ST_MapAlgebraExpr function. Hence, for entries that have more than two hints, the overlay process has to be carried out more than once. In the first instance, two hints' rasters can be overlaid and in the second instance, the resultant raster is overlaid with the third hint's raster to produce a 'resulting raster 2'. This process can be repeated until all the hints' rasters are overlaid to produce a 'final resulting raster'. 
For any hint $h \in e$, where $e$ is the gazetteer entry, the resulting raster for the entry is generated by taking the product two hint rasters and can be represented as

$$
\operatorname{rast}(e)=\prod_{h \in e} \operatorname{rast}(h)
$$

where rast $(e)$ is the resulting raster of an entry $e$ and $\operatorname{rast}(h)$ is the hint raster for any hint $h \in e$.

Figure 5.18 shows how a rast(e) was created by overlapping two rast $(h)$. Using the rasters chosen for overlay in Section 5.7 for different hint types i.e., distance-direction from the reference point, distance along/from the reference object and between-two-points, we carried out raster overlay for 48 entries using 96 rast $(h)$ covering different hint types.

\subsubsection{Evaluation of results}

For each of our sample entries, we overlaid all the relevant rast $(h)$ to create rast(e). In our analysis, we found that of the total 48 entries, only two entries had an inconsistent hint each and hence no rast(e) could be created for those entries. Thus, it can be said that $96 \%$ of the sample entries had consistent hints. We further analysed if the locality was found to be located in all resulting rasters or not. We found that 39 of 46 i.e., $85 \%$ rast (e) carried the target locality. For the rest of seven rast (e), the overlay was possible but target locality did not fall in their intersection area.

\subsubsection{Accuracy of location}

We also evaluated our rast (e) to gauge how accurately they have been able to predict the location of a target locality. We work here for a number of entries for which the location of target locality is known. Thus, in rast(e) the cell vale in which the target locality is located is denoted by target $(e)$ and its value is denoted by rast $(e)(\operatorname{target}(e))$. For simplicity sake we denote it by $\operatorname{tcv}(e)$ which gives us the target cell's value for an entry. Now, for the same rast(e) there exists a cell with maximum probability value, which is also the predicted location of the target locality for that entry. We denote the value of the maximum cell value by $\operatorname{mcv}(e)$. Since, for seven entries the target locality fell outside the rast (e), we could only do this analysis for 39 of 46 entries.

For best results, $\operatorname{tcv}(e)$ should be close to $\operatorname{mcv}(e)$. Thus, we analyse for our sample entries, the deviation of $t c v(e)$ from $\operatorname{mcv}(e)$. A smaller deviation implies that the actual location is close to the predicted location. We measured this deviation as 'percent deviation' for each rast (e). The percent deviation is a statistical measure often used to test the accuracy of results in reference to a point in the data. Most often, this reference point is the mean value. In our case however, we wish to measure the 


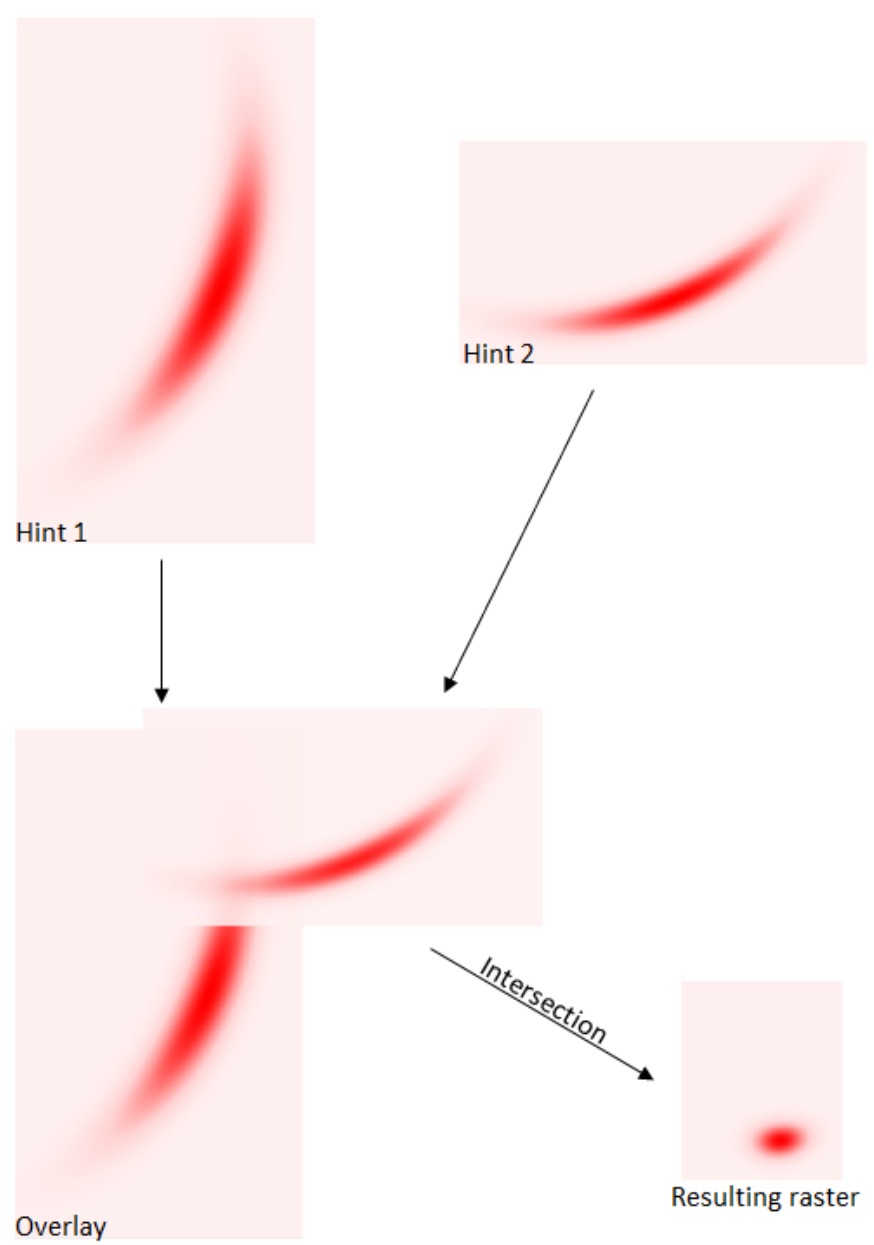

Figure 5.18 Illustration to show creation of resulting raster after intersection of two overlapping hint rasters

percent deviation of the actual location from the predicted location in the resultant raster and it is calculated as follows,

$$
p d(e)=100\left(\frac{\operatorname{tcv}(e)-m c v(e)}{m c v(e)}\right),
$$

where $p d(e)$ is percent deviation in rast (e). In Figure 5.19, it can be seen that for $31 \%$ of the sample entries, the $p d(e)$ of $\operatorname{tcv}(e)$ from $\operatorname{mcv}(e)$ was less than $10 \%$. Further, for $64 \%$ of our sample entries, the $p d(e)$ was less than $50 \%$.

Figure 5.20, shows three resulting rasters for which $p d(e)$ values are $3 \%, 46 \%$ and $100 \%$, respectively.

However, it is also important to notice that $36 \%$ of the entries have a higher $p d(e)$ between $50 \%-100 \%$. This prompted us to look further 


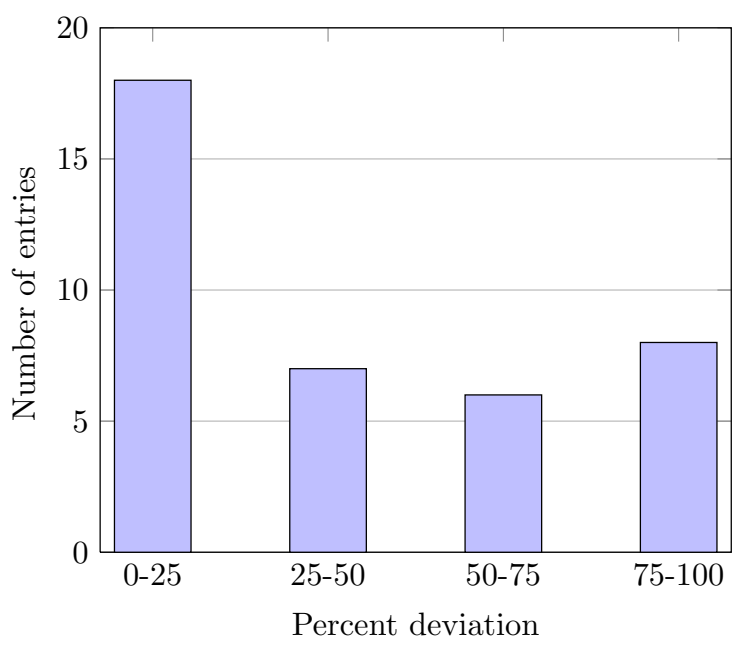

Figure 5.19 Percent deviation in probability values over 39 of 46 resulting rasters

(a)

(b)

(c)

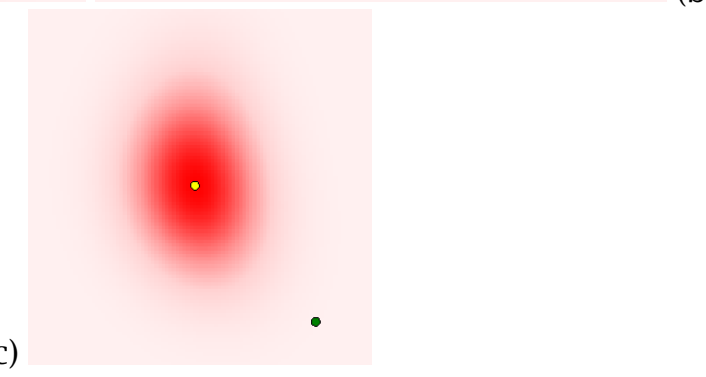

Figure 5.20 Percent deviation of $\operatorname{tcv}(e)$ (in green) from $m c v(e)$ (in yellow) (a) minimum deviation (3\%), (b) mid-level deviation (46\%), (c) maximum deviation (100\%)

into our resultant rasters and we found that the $p d(e)$ scores were particularly high for those entries that also had hints of the type 'along the line feature'. A closer examination of the rasters made for this hint type revealed that in these rasters, high probability density was assigned throughout the line feature and as soon as the point moves away from line feature, the probability densities fell sharply. This pattern of probability distribution affected the probability calculation in rast $(e)$ as 


\section{o}

Figure 5.21 Percent deviation of $\operatorname{tcv}(e)$ (in green) from $m c v(e)$ (in yellow) is $51 \%$

the absolute probability densities of the overlaying rast $(h)$ are multiplied in rast (e). As an example, Figure 5.21 shows one such rast $(h)$ in which even though the $t c v(e)$ is close to $m c v(e)$ and closer to the line feature, the $p d(e)$ is about $52 \%$. Thus, it can be inferred that there is a skew in $p d(e)$ scores. To get a better understanding on location accuracy we also measured the distances between the $\operatorname{tcv}(e)$ and $m c v(e)$ for all relevant rast (e). For this analysis, all 46 rast(e) were studied since this does not

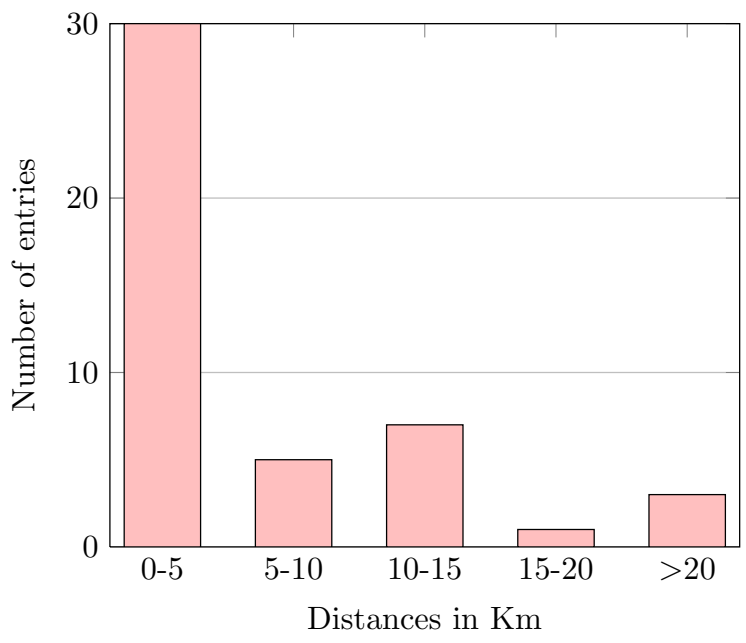

Figure 5.22 Distances between $\operatorname{tcv}(e)$ and $m c v(e)$ over 46 resulting rasters

require the presence of target locality within the raster. The following graph (Figure 5.22), shows distances between actual location $(\operatorname{tcv}(e))$ from the predicted location $(m c v(e))$. As shown in the graph, for nearly $65 \%$ of the 46 entries the predicted location is within $5 \mathrm{~km}$ of the actual location. As expected for the seven rast $(e)$ for which the target locality is outside the rast $(e)$, the distance deviation was found to be very high. In fact all the entries with a distance deviation of more than $14 \mathrm{~km}$ are 
the ones for which the target locality is outside the rast(e). Thus, of the remaining 39 rast(e), 30 entries have a distance deviation of less than $5 \mathrm{~km}$ and maximum deviation is $14 \mathrm{~km}$.

\subsection{Overlaying rasters with vector model}

So far, in this chapter, we have created raster representations of hints and overlaid them to create resultant rasters. We also evaluated hint consistency and accuracy of predicting location of the target locality. For the same entries, in addition to hint represented by rasters, there often exists a directional containment hint. Those hints are represented in vector models, which were discussed in Chapter 4. Hence, in this section, we overlay the raster representation of hints in an entry with the vector models of hint in the same entry. This allows us to verify the consistency of hints represented in different spatial formats, i.e., raster and vector.

\subsubsection{Resultant raster with vector model}

We consider entries that have more than one hint represented in raster format and also have the directional containment hint. Thus, for these entries, the resultant raster will be overlaid with the vector model of its directional containment hint.

On doing so, we found that for almost $85 \%$ of entries, resultant rasters successfully overlaid with vector models, implying that all the hints in those entries are mutually consistent. For the other entries, one of the two representations represents inconsistency. Since our resultant rasters have already been verified for consistency in Section 5.8.1, hence only the hints represented by vector models can be inconsistent for these entries. This was also confirmed after a visual verification. We further verified if the point of predicted location, in the raster, falls within the vector models of directional containment or not. Of the entries, for which resultant rasters could be overlaid with their vector models, $71 \%$ of the rasters had points of predicted location falling within the directional containment models. This is not to say that the other overlays are inaccurate, but just not as accurate as other $71 \%$. That is because their point of predicted location falls just outside the vector models and we do not expect the target locality to fall exactly at predicted location. In Section 5.8.1.1, we also analysed the levels of distance deviation of predicted locations from observed locations. Figure 5.23, shows a successful overlay of a resultant raster with the directional sector of the vector model, which is described in an entry to be in 'southern central Rio de Janeiro.' It also shows the predicted location of target locality as a point. 


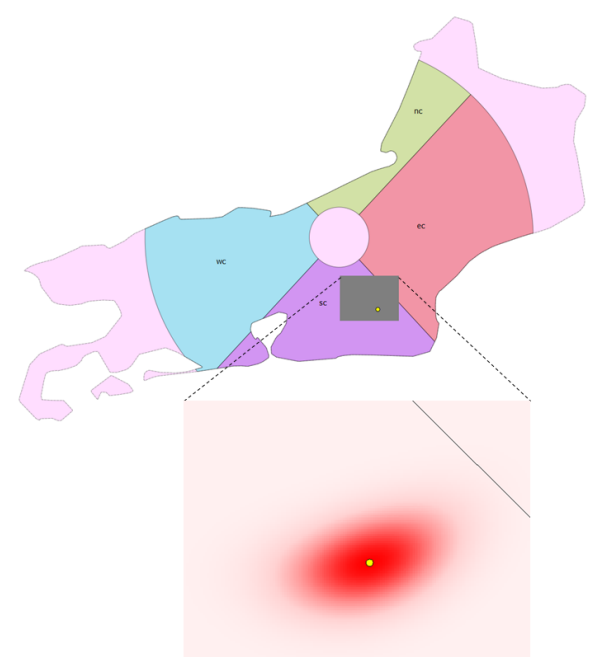

Figure 5.23 A successful overlay of a resulting raster with vector model for a single entry which described a locality to be in southern central Rio de Janeiro'

\subsubsection{Single rasters with vector models}

There are also entries that have only one hint that can be represented using a raster but they also have the directional containment hint represented using vector-based models. For those entries, we do not have resultant raster, so we overlay the single rasters of those hints with the vector models. The results indicate that $86 \%$ of entries have mutually consistent hints as their respective spatial representations could be successfully intersected. These results are slightly better than the results of previous section because in the current case, the extents of the rasters are larger than the resultant rasters. However, the area of certainty in the resultant rasters is much better defined and comes with a high level of confidence because the hints in an entry are cross-validated in the process of overlaying. Again, the cell with the predicted location of target locality was converted to a point geometry and the same was used to verify if it falls within the directional containment sector of the vector model or not. Of the successful overlays, $63 \%$ of the rasters' points of predicted location fell within the respective directional sector of the vector model. For a single entry, which is described to be 'in eastern Amazonas', Figure 5.24 shows a successful overlay of a raster with the respective direction sector.

\subsection{Summary}

In this chapter, we studied the uncertainty that exists in spatial hints and we also provided the approach to model these hints using probabilitybased rasters. The raster creation for different hint types was customised 


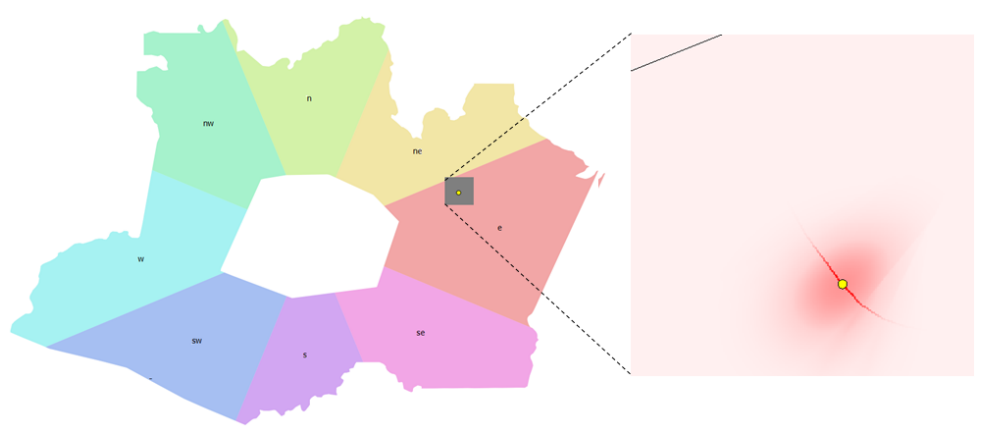

Figure 5.24 A successful overlay of a single hint raster with its vector model for an entry

because of different information available in the hint types. We discussed how rasters were created and how their parameters were optimised for most optimal rasters. These rasters were then overlaid to create resultant rasters that have a much better defined area of associated uncertainty within which the target locality can be expected to fall.

To evaluate the performance of these resultant rasters, we measured the deviation of observed location of the target locality from its predicted location. For $64 \%$ of the resultant rasters, this deviation was less than $50 \%$. As a next step, the resultant rasters were overlaid over the vector models that were created for the directional containment hints. For $85 \%$ of the cases, the hints represented by the resultant rasters and the vector models were found to be consistent and for $71 \%$ of cases, the predicted location of target locality also fell within the vector models. We also overlaid the single rasters with vector models for entries, where only one hint could be represented with rasters. For those entries, $86 \%$ cases had mutually consistent hints and for $63 \%$ entries, the predicted location of target locality fell within the vector models.

Having come so far, we believe that our analytical approach of modeling uncertainty using probability-based rasters can be successfully used to identify that area in space where the target locality is expected to fall.

In the next chapter, we provide a synthesis of this thesis, which includes conclusion, limitations and recommendations for future work. 



\section{Synthesis}

This chapter provides conclusions of the work presented in this thesis. Like any other research project, that for this thesis also has limitations and they are discussed here. We end this chapter by recommending future areas of work and include suggestions to improve the current work and suggest additional research ideas where our analytical approach can be applied.

\subsection{Conclusions}

In the past (i.e., in the pre-GPS days), when the technique to collect and share information were limited, locations and their names were recorded as textual descriptions. It is believed that natural history expeditions over 250 years have collected over a billion biological specimens, all with ancillary information about the locality where the specimen was collected. However, this information does not always provide coordinates and this is one of the barriers in any spatial, statistical or historical analysis (Beaman et al., 2004) of those sources. Today, most of this locational information on specimens is available in the form of printed gazetteers. We studied one such gazetteer known as the 'Ornithological gazetteer of Brazil', a two-volume publication from 1991. We propose an approach to find geocodes of sites mentioned in the gazetteer such that the information from it can be properly utilised. The gazetteer, authored by the renowned ornithologists Paynter and Traylor - contains 8000 entry descriptions covering roughly 6000 sites in Brazil. Since Brazil contributes significantly to the Global Biodiversity Information Facility (GBIF), any investigation into geocoding entries in this gazetteer can be helpful in GBIF database enrichment. Paynter and Traylor also authored ten more such gazetteers for South America. It may be possible to investigate these gazetteers using our approach and tools because they are expected to have similar textual structure and style of writing.

As a starting point to investigation of the gazetteer, we scanned and stored it in a digital form in the database. The textual structure of the descriptions in the gazetteers is easier to understand by humans but is difficult to exploit by machines, yet it still allows, for various spatial analyses or computations to be carried out. However, before any 
computation can be carried out, an extraction of information from these hints into structured entities is required. Since these descriptions are represented as natural language text, they also carry uncertainty that need to be identified and understood. With this in mind, we laid down the following objectives as part of this research project:

1. To understand the textual structure of gazetteer entries and identify hints and their components that can be exploited spatially,

2. To identify and exploit syntactic structure of hints by developing patterns for extraction and storage of hints in a suitable data structure for efficient storage and retrieval,

3. To identify types of uncertainty that occur in our spatial hints,

4. To develop vector-based or raster-based spatial representations for spatial hints,

5. To predict area of certainty by combining raster-based and vectorbased spatial representations for various entry descriptions to derive an area of certainty in which the locality is expected to fall.

In the process of understanding gazetteer entries, typical contents of an entry were identified, such as place name, state name, geocode (if mentioned) and a description. Each description further carries various spatial hints in the form of elevation, place name, reference features, distance, direction and others. In Chapter 2, we identified various such types of spatial hint present in gazetteer descriptions. These spatial hints carry important information and to do any spatial computation or analysis they need to be extracted and stored in a structured format. This was discussed in Chapter 3. In that chapter, we also explained implementation of extracting information from hints using techniques from Natural Language Processing (NLP) and Information Extraction (IE). Since our descriptions have compound names, abbreviations and extra punctuation, they were normalised to derive correct parse trees. After that, the descriptions were parsed through the NLP pipeline where the syntactic structure was produced in the form of a parse tree. These parse trees were then analysed to identify hint-specific subtrees carrying information. To extract this information, we used the tree query language (Tregex) to develop hint-specific patterns. The parse trees and the developed patterns were then used to find matches in the parse tree against the hint patterns under consideration. Once a match was found, our hint extraction algorithm extracted the information and stored it in a predefined data structure in the database.

To evaluate the performance of our developed patterns, recall scores were used and we found that patterns for most of the hint types had a good recall score of 0.72 on average. Finally, the extracted information was used to create the vector-based and raster-based spatial representation of the hints in further chapters.

In Chapter 4, we worked on 'directional containment' hints for spatial representation as these are the most frequently occurring (54\%) spatial hints in our gazetteer descriptions. In this chapter, we created 
vector-based spatial extent models that help to interpret directional containment phrases for each category of direction types, i.e., full directions, half-directions, and extreme directions. We studied how different spatial extent models can be created based on model characteristics, i.e., placement, shape and size. Then we created models for all the direction types, namely, full directions, half-directions and extreme directions. Since these models are used for mapping descriptions, their performance was evaluated by using recall and precision scores. We found that for full directions, spatial extent models with 'mass box centre/hull central sector/angular outer sector' are the optimal choice of representation. In half-directions, 'region mass centre/circle-central/angular outer sector' were found optimal for central half-directions, whereas for non-central half-directions, 'circle-centre/circle-central/angular outer sectors' were found to be the optimal choice. For extreme directions, the 'region mass centre/area-based circle' approach with angular outer sector as optimal choice. In Chapter 5, we looked at some other hint types that were not covered in Chapter 4 and created spatial representations for them.

In Chapter 5, we identified the types of uncertainty in our spatial hints and how these can be modeled using probability models. For different hint types, raster-based spatial representations were created at different levels of uncertainty. The best performing rasters with optimised parameters for raster creation were also identified per hint type. Using the rasters created with optimised parameters, raster-overlay was carried out for hints within an entry to create resultant rasters that define the area of associated uncertainty, within which the target locality of an entry is expected to fall. These resultant rasters were further evaluated on their accuracy in predicting the location of the target locality. For $64 \%$ of the resultant rasters the probability value at the observed location of the locality was within a percent deviation score of $50 \%$ from the predicted location. Further, nearly, $60 \%$ of entries predicted location is within $5 \mathrm{~km}$ distance of observed location. The two points i.e., the predicted and observed location, were closest at about 500 meters and farthest at $41 \mathrm{~km}$. The resultant rasters were further overlaid with vector models of representation for directional containment hints, as developed in Chapter 4. It was found that for 15\% of the cases, the directional containment hint was inconsistent and the rasters could not be successfully overlaid with the vector models. Furthermore, it was found that for $71 \%$ of the rasters, the predicted location of the target locality fell within the vector model. We also studied the entries with only single hint other than the directional containment hint. Thus, the raster of the former hint was overlaid with the vector model of the latter hint. This time, for $86 \%$ of entries, rasters were successfully overlaid with vector models. Further, for $63 \%$ of those entries, the predicted location of target locality fell within the vector model.

Using the results of our extraction process and vector and rasterbased spatial representations, one can identify the areas of associated uncertainty where the target localities, as described in their entries, can be located. The approach adopted in this thesis is generic and can be 
applied to other similar text sources. However, it must be mentioned that there are limitations to this work and that there are topics deserving future work in the future. In the following sections, we are pointing out those limitations and recommendations for future work.

\subsection{Limitations}

The following are some of the limitations of this research project.

- The process of extracting spatial hints from our corpus is a semiautomated process since the patterns had to be constructed for each hint type. It can be faster if an annotated corpus in the geographic domain is available to train the parser. Alternatively, a parser from the geographic domain can also be useful for automating the extraction process.

- Generally, prominent geographic features such as coastlines, large rivers, and mountain ranges appear to affect entry descriptions, e.g., 'northwestern Amazonian Pará' in Brazil is typically associated with that part of state that is northwest of the Rio Amazonas. Similarly, 'coastal central Maranhão' is associated with the central part of coast line of that state. Our work observed these (cognitive) effects but did not study or address then in detail.

- The river data available to us was primarily on the major rivers in Brazil while our entries also had hints in reference to smaller rivers, streams and tributaries for which we did not have enough line geometries. This limited our sample of analysis for 'along the line feature' hint type.

- The function developed for modeling along the line feature hint type for distribution of probability, can be improved. As we found in Chapter 5, the probability is very high on the line feature and drops sharply as soon as the point moves away from the line. The reduction in these probabilities should be more gradual. This affected the probabilities in the resultant rasters, thereby bringing down the percent deviation score. However, even as they are, these rasters are perfectly capable of determining the area of certainty.

- Since raster creation is a highly time consuming process, our research had to work with a sample of entries and we could work with only nine rasters per hint for some spatial hint types. More choice of parameters would help in better optimization.

- In this project, we identified eight types of spatial hints that exist in our entry descriptions. However, we studied five of them and created spatial representations of four. Even so, the hints we covered are the most frequently occurring and most important types of hints in our corpus. 


\subsection{Recommendations for future work}

In this section, we identify those aspects of the project that still need further study and those topics to which, our project approach can be applied.

- An extraction tool specific to the geographic domain should be developed that not only covers place names, as available in existing NER, but also covers the spatial relations between two places. For example, there are many extraction tools available in the medicine and crime domains.

- We created spatial representations for some hints, but not all. Other hints, such as between a point and a line, or between line and another line feature, can be studied to model their uncertainty.

- Using our analytical approach of creating spatial representation of spatial hints, all the entries in our gazetteer can be modeled and geocodes of the target locality can be predicted for entries with unknown geocode.

- Another extension of our work can be to work on ten other gazetteers for South America, written by the same authors, since the style of reporting the entries is similar in all those gazetteers. The information thus created, can be contributed to GBIF or the Avian Knowledge Network (AKN).

- The approach of our work can find application in some other domains. For example, major events or natural disasters are often reported as '64km WSW of Panguna, Papua New Guinea' or '113km SE of Amsterdam Island' as mentioned on USGS website ${ }^{1}$. Similarly, various news agencies often report place(s) where event(s) have occurred like 'riots in southern part of state'.

\footnotetext{
${ }^{1}$ http://earthquake.usgs.gov/ (last accessed on Oct. 17, 2013).
} 



\section{Bibliography}

Abascal-Mena, R. and López-Ornelas, E. Geo information extraction and processing from travel narratives. In Proceedings of ELPUB, pages 363-373. 2010.

Abuzaid, A. H., Mohamed, I. B., and Hussin, A. G. Boxplot for circular variables. Computational Statistics, pages 1-12, 2011.

Adeva, J. J. Translating vernacular terms into geographical locations. In Sample, J., Shaw, K., Tu, S., and Abdelguerfi, M., editors, Geospatial Services and Applications for the Internet, volume 3693 of Springer, pages 135-153. Springer, US, 2008.

Aho, A. V. and Ullman, J. D. The theory of parsing, translation, and compiling. Prentice-Hall, Inc., Upper Saddle River, NJ, USA, 1972. ISBN 0-13-914556-7.

AOU, 2002. Elliott Coues Award, 2001: Raymond A. Paynter, Jr., and Melvin A. Traylor, Jr. The Auk, The American Ornithologists' Union, 119(1):281-282, 2002.

Appelt, D. E., Hobbs, J. R., Bear, J., Israel, D., and Tyson, M. Fastus: A finite-state processor for information extraction from real-world text. In Proceedings of the International Joint Conference on Artificial Intelligence (IJCAI), pages 1172-1178, 1993.

Arpinar, I. B., Sheth, A. P., Ramakrishnan, C., Usery, E. L., Azami, M., and Kwan, M.-P. Geospatial ontology development and semantic analytics. Transactions in GIS, 10(4):551-575, 2006.

Batista, D. S., Silva, M. J., Couto, F. M., and Behera, B. Geographic signatures for semantic retrieval. In Proceedings of the 6th Workshop on Geographic Information Retrieval, GIR '10, pages 19:1-19:8, New York, NY, USA, 2010. ACM. ISBN 978-1-60558-826-1.

Beaman, R., Wieczorek, J., and Blum, S. Determining space from place for natural history collections. D-lib Magazine, 10(5):1082-1090, 2004.

Beaubouef, T., Petry, F. E., and Ladner, R. Spatial data methods and vague regions: A rough set approach. Applied Soft Computing, 7(1):425-440, 2007.

Bies, A., Ferguson, M., Katz, K., and MacIntyre, R. Bracketing guidelines for treebank ii style penn treebank project. Technical report, 
1995. URL http://www.1dc.upenn.edu/Catalog/docs/LDC99T42/ prsguid1.pdf.

Bittner, T. and Stell, J. G. Rough sets in approximate spatial reasoning. In Second International Conference on Rough Sets and Current Trends in Computing, volume 2005 of Lecture Notes in Computer Science (LNCS), pages 445-453. Springer-Verlag, 2000.

Bloch, I. Spatial reasoning under imprecision using fuzzy set theory, formal logics and mathematical morphology. International Journal of Approximate Reasoning, 41(2):77 - 95, 2006.

Booth, T. L. and Thompson, R. A. Applying probability measures to abstract languages. IEEE Transactions on Computers, C-22(5):442-450, 1973.

Clementini, E. and di Felice, P. A spatial model for complex objects with a broad boundary supporting queries on uncertain data. Data \& Knowledge Engineering, 37(3):285-305, June 2001.

Clementini, E. and Felice, P. D. Approximate topological relations. International Journal of Approximate Reasoning, 16(2):173-204, 1997.

Cohn, A. G. and Gotts, N. M. Geographic objects with indeterminate boundaries, chapter The 'egg-yolk' representation of regions with indeterminate boundaries, pages 171-187. Number 2 in GISDATA. Taylor \& Francis, London, 1996.

Cohn, A. G., Bennett, B., Gooday, J., and Gotts, N. M. Qualitative spatial representation and reasoning with the region connection calculus. GeoInformatica, 1(3):275-316, 1997.

Collier, N., Park, H. S., Ogata, N., Tateishi, Y., Nobata, C., Ohta, T., Sekimizu, T., Imai, H., Ibushi, K., and Tsujii, J. The genia project: corpusbased knowledge acquisition and information extraction from genome research papers. In In Ninth Conference of the European Chapter of the Association for Computational Linguistics (EACL-99, pages 271-272, 1999.

Comber, A. J., Fisher, P. F., Harvey, F., Gahegan, M., and Wadsworth, R. Using metadata to link uncertainty and data quality assessments. In Riedl, A., Kainz, W., and Elmes, G. A., editors, Progress in Spatial Data Handling, pages 279-292. Springer Berlin Heidelberg, 2006. ISBN 978-3-540-35588-5.

Cowie, J. and Lehnert, W. Information extraction. Commun. ACM, 39(1): 80-91, January 1996. ISSN 0001-0782.

de By, R. A. A funny distance computation and ... the power of spatial SQL. Technical report, Faculty of Geo-information Science \& Earth Observation (ITC), University of Twente, 2012. URL http://gip.itc. n1/defau1t/sites/default/files/average_distance.pdf.

Dehak, S. M. R., Bloch, I., and Maitre, H. Inference of directional spatial relationship between points: a probabilistic approach. In Image Processing, 2001. Proceedings. 2001 International Conference on, volume 3, pages 704-707, 2001. 
Dehak, S. M. R., Bloch, I., and Maitre, H. Spatial reasoning with incomplete information on relative positioning. Pattern Analysis and Machine Intelligence, IEEE Transactions on, 27(9):1473-1484, 2005.

Dhillon, I. S. and Sra, S. Modeling data using directional distributions. Technical report, 2003.

Drymonas, E. and Pfoser, D. Geospatial route extraction from texts. In Proceedings of the 1st ACM SIGSPATIAL International Workshop on Data Mining for Geoinformatics, DMG '10, pages 29-37, New York, NY, USA, 2010. ACM. ISBN 978-1-4503-0430-6.

Egenhofer, M. J., Mark, D. M., and Herring, J. The 9-intersection: Formalism and its use for natural-language spatial predicates. Technical Report 94-1, National Center for Geographic Information \& Analysis (NCGIA), 1994.

Feldman, R. Information extraction, theory and practice, 2006. URL http://pluto. huji .ac.i1/ rfe1dman/ICML2006.pdf.

Fisher, P., Comber, A., and Wadsworth, R. Approaches to Uncertainty in Spatial Data, pages 43-59. ISTE, 2006. ISBN 9780470612156.

Fisher, P. F. Models of uncertainty in spatial data. In Longley, P. A., Goodchild, M. F., and Maguire, D. J., editors, Geographic Information Systems: Principles and Technical Issues 2nd edition, pages 191-205, New York, U.S.A, 1999. John Wiley.

Forbes, C., Evans, M., Hastings, N., and Peacock, B. Statistical Distributions. John Wiley \& Sons, Inc, U.S.A, 4th edition, 2011. ISBN 978-0-470-390634.

Frank, A. U. Qualitative spatial reasoning about distances and directions in geographic space. Journal of Visual Languages \& Computing, 3(4): 343-371, 1992.

Frank, A. U. Qualitative spatial reasoning: Cardinal directions as an example. International Journal of Geographical Information Systems, 10(3):269-290, 1996.

Freksa, C. Using orientation information for qualitative spatial reasoning. In Frank, A., Campari, I., and Formentini, U., editors, Theories and Methods of Spatio-Temporal Reasoning in Geographic Space, LNCS \#639, pages 162-178, 1992.

Friedman, C. Towards a comprehensive medical language processing system: methods and issues. In In Proceedings of AMIA Annu Fall Symp, 1997.

Fu, P. and Sun, J. Web GIS Principles and Applications. ESRI Press, Redlands, California, U.S.A., 2011.

Gahegan, M. and Ehlers, M. A framework for the modelling of uncertainty between remote sensing and geographic information systems. \{ISPRS\} Journal of Photogrammetry and Remote Sensing, 55(3):176 - 188, 2000. ISSN 0924-2716. 
Gaile, G. L. and Burt, J. E. Directional statistics. Concepts and techniques in modern geography; no. 25. Geo Abstracts Ltd., Norwich, U.K, 1980. ISBN 0860940322.

Gelernter, J. and Mushegian, N. Geo-parsing messages from microtext. Transactions in GIS, 15(6):753-773, 2011.

Godoy, J., Atkinson, J., and Rodriguez, A. Geo-referencing with semiautomatic gazetteer expansion using lexico-syntactical patterns and coreference analysis. International Journal of Geographical Information Science, 25(1):149-170, 2011.

Goldberg, D. W., Wilson, J. P., and Knoblock, C. A. From text to geographic coordinates: the current state of geocoding. URISA Journal, 19(1):3347, 2007.

Goodchild, M. F. and Zhang, J. Uncertainty in Geographical Information Research monographs in geographic information systems. Taylor \& Francis, 2002. ISBN 9780203471326.

Goyal, R. K. and Egenhofer, M. J. Consistent queries over cardinal directions across different levels of detail. In Proceedings 11th International Workshop on Database and Expert Systems Applications, Greenwich, U.K., pages 876-880, 2000.

Grishman, R. and Sundheim, B. Message understanding conference-6: a brief history. In Proceedings of the 16th conference on Computational linguistics - Volume 1, COLING '96, pages 466-471, Stroudsburg, PA, USA, 1996. Association for Computational Linguistics.

Guo, Q., Liu, Y., and Wieczorek, J. Georeferencing locality descriptions and computing associated uncertainty using a probabilistic approach. International Journal of Geographical Information Science, 22:10671090, January 2008.

Haar, R. Computational models of spatial relations. Computer Science technical report series. Computer Science Center, University of Maryland, 1976.

Habib, M. B. and van Keulen, M. Named entity extraction and disambiguation: The reinforcement effect. In Proceedings of the 5th International Workshop on Management of Uncertain Data, MUD 2011, Seatle, USA, 2011.

Hagège, C., Sándor, A., and Schiller, A. Linguistic processing of biomedical texts. In Ranchhod, E. and Mamede, N., editors, Advances in Natural Language Processing, volume 2389 of Lecture Notes in Computer Science, pages 197-207. Springer Berlin Heidelberg, 2002. ISBN 978-3-540-43829-8.

Hill, L. L. Georeferencing: The Geographic Associations of Information, page 260 pp. The MIT Press, Cambridge, London, U.K, 2006.

Huffman, S. B. Learning information extraction patterns from examples. In In IJCAI, workshop on new approaches to learning for Natural Language Processing, pages 246-260. Springer, 1995. 
Hunter, G. J. Managing uncertainty in gis. In Longley, P. A., Goodchild, M. F., Maguire, D. J., and Rhind, D. W., editors, Geographical Information Systems. John Wiley and Sons Ltd., 1999.

Hunter, G. J. and Goodchild, M. F. Managing uncertainty in spatial databases: Putting theory into practice. In In: Proc. of Urban and Regional Information Systems Association, pages 1-14, 1993.

Hunter, G. J. and Goodchild, M. F. Communicating uncertainty in spatial databases. Transactions in GIS, 1(1):13-24, 1996. ISSN 1467-9671.

Jackson, P. and Schilder, F. Natural language processing: Overview. Encyclopedia of Language \& Linguistics, 13:503-518, 2006.

Jones, C. B., Purves, R. S., Clough, P. D., and Joho, H. Modelling vague places with knowledge from the web. International Journal of Geographical Information Science, 22(10):1045-1065, 2008.

Kiiveri, H. T. Assessing, representing and transmitting positional uncertainty in maps. International Journal of Geographical Information Science, 11(1):33-52, 1997.

Kim, J. T. and Moldovan, D. I. Acquisition of linguistic patterns for knowledge-based information extraction. Knowledge and Data Engineering, IEEE Transactions on, 7(5):713-724, 1995.

Klement, E. P., Mesiar, R., and Pap, E. Triangular norms. position paper i: basic analytical and algebraic properties. Fuzzy Sets and Systems, 143 (1):5-26, 2004.

Leidner, J. L., Sinclair, G., and Webber, B. Grounding spatial named entities for information extraction and question answering. In Proceedings of the HLT-NAACL 2003 workshop on Analysis of geographic references - Volume 1, HLT-NAACL-GEOREF '03, pages 31-38, Stroudsburg, PA, USA, 2003. Association for Computational Linguistics.

Levinson, S. C. Space in Language and Cognition: Explorations in Cognitive Diversity. Cambridge University Press, U.K., 2003.

Levy, R. and Andrew, G. Tregex and tsurgeon: tools for querying and manipulating tree data structures. In Proceedings of the 5th International Conference on Language Resources and Evaluation, LREC '06, 2006.

Ligozat, G. "corner" relations in allen's algebra. Constraints, 3(2/3): 165-177, 1998.

Liu, Y., Wang, X., Jin, X., and Wu, L. On internal cardinal direction relations. In Cohn, A. and Mark, D., editors, Spatial Information Theory, volume 3693 of Lecture Notes in Computer Science, pages 283-299. Springer Berlin/Heidelberg, 2005.

Liu, Y., Guo, Q. H., Wieczorek, J., and Goodchild, M. F. Positioning localities based on spatial assertions. International Journal of Geographical Information Science, 23(11):1471-1501, 2009.

Ljunglöf, P. and Wirén, M. Syntactic parsing. In Indurkhya, N. and Damerau, F. J., editors, Handbook of Natural Language Processing, Second Edition. CRC Press, Taylor and Francis Group, Boca Raton, FL, 2010. ISBN 978-1420085921. 
Longley, P. A., Goodchild, M. F., Maguire, D. J., and Rhind, D. W. Geographic Information Systems and Science. John Wiley and Sons, New York, U.S.A, 2nd edition, 2005.

Lucas, C. Multi-criteria modelling and clustering of spatial information. International Journal of Geographical Information Science, 26(10):18971915, 2012.

Ma, Y., Kalashnikov, D. V., and Mehrotra, S. Toward managing uncertain spatial information for situational awareness applications. IEEE Transactions on Knowledge and Data Engineering, 20(10):1408-1423, October 2008.

Mardia, K. V. and Jupp, P. E. Directional Statistics. John Wiley \& Sons, Ltd., Chichester, London, U.K, 2nd edition, 2000.

Mark, D. and Egenhofer, M. J. Calibrating the meanings of spatial predicates from natural language: Line-region relations. In Proceedings of Spatial Data Handling, Edinburgh, Scotland, Sept 1994.

Moens, M. F. Information Extraction: Algorithms and Prospects in a Retrieval Context. Springer, Dordrecht, Netherlands, 2006. ISBN 978-14020-4987-3.

Moore, D., McCabe, G. P., and Craig, B. Introduction to the Practice of Statistics. W. H. Freeman and company, New York, U.S.A, 2012.

Moratz, R., Nebel, B., and Freksa, C. Qualitative spatial reasoning about relative position. In Freksa, C., Brauer, W., Habel, C., and Wender, K. F., editors, Spatial Cognition III, volume 2685 of Lecture Notes in Computer Science, pages 385-400. Springer Berlin Heidelberg, 2003. ISBN 978-3-540-40430-9.

Moratz, R., Dylla, F., and Frommberger, L. A relative orientation algebra with adjustable granularity. In Proceedings of the workshop on agents in real-time and dynamic environments (IJCAI), 2005.

Muslea, I. Extraction patterns for information extraction tasks: A survey. In In AAAI-99 Workshop on Machine Learning for Information Extraction, pages 1-6, 1999.

Napolitano, G., Fox, C., Middleton, R., and Connolly, D. Pattern-based information extraction from pathology reports for cancer registration. Cancer Causes and Control, 21:1887-1894, 2010. ISSN 0957-5243.

Ni, J., Ravishankar, C., and Bhanu, B. Probabilistic spatial database operations. In Hadzilacos, T., Manolopoulos, Y., Roddick, J., and Theodoridis, Y., editors, Advances in Spatial and Temporal Databases, volume 2750 of Lecture Notes in Computer Science, pages 140-158. Springer Berlin / Heidelberg, 2003.

NIST. Automatic content extraction (ace) program. Technical report, 1998.

Papadias, D. and Egenhofer, M. J. Algorithms for hierarchical spatial reasoning. GeoInformatica, 1(3):251-273, 1997. ISSN 1384-6175. 
Paynter, R. A., Jr. and Traylor, M. A., Jr. Ornithological Gazetteer of Brazil, volume A-M. Harvard University, Museum of Comparative Zoology, Bird Department, Cambridge, Ma., U.S.A., 1991a.

Paynter, R. A., Jr. and Traylor, M. A., Jr. Ornithological Gazetteer of Brazil, volume N-Z. Harvard University, Museum of Comparative Zoology, Bird Department, Cambridge, Ma., U.S.A., 1991b.

Peuquet, D. J. and Zhang, C. X. An algorithm to determine the directional relationship between arbitrarily-shaped polygons in the plane. Pattern Recogn., 20(1):65-74, January 1987.

Randell, D. A., Cui, Z., and Cohn, A. G. A spatial logic based on regions and connection. In Nebel, B., Rich, C., and Swartout, W., editors, Proceedings of the Third International Conference on Principles of Knowledge Representation and Reasoning (KR'92), pages 165-176. Morgan Kaufmann, San Mateo, California, 1992.

Riloff, E. Automatically generating extraction patterns from untagged text. In Proceedings of the thirteenth national conference on Artificial intelligence - Volume 2, AAAI'96, 1996.

Riloff, E. and Lorenzen, J. Extraction-based text categorization: Generating domain-specific role relationships automatically. In Strzalkowski, T., editor, Natural Language Information Retrieval, volume 7 of Text, Speech and Language Technology, pages 167-196. Springer, Netherlands, 1999. ISBN 978-90-481-5209-4.

Rowe, R. J. Elevational gradient analyses and the use of historical museum specimens: a cautionary tale. Journal of Biogeography, 32(11):18831897, November 2005. ISSN 0305-0270.

Roy, A. J. and Stell, J. G. Spatial relations between indeterminate regions. International Journal of Approximate Reasoning, 27(3):205-234, September 2001.

Sarawagi, S. Information extraction. Found. Trends databases, 1(3): 261-377, March 2008. ISSN 1931-7883.

Schneider, M. Modelling spatial objects with undetermined boundaries using the real/rose approach. Geographic objects with indeterminate boundaries, 2:141-152, 1996.

Schwering, A. Semantic similarity of natural language spatial relations. In Conference on Artificial Intelligence and Simulation of Behaviour (AISB07): Artificial and Ambient Intelligence, pages 491-496, 2007.

Sekine, S. and Nobata, C. Definition, dictionaries and tagger for extended named entity hierarchy. In LREC. European Language Resources Association, 2004.

Shariff, A. R., Egenhofer, M., Egenhofer, M., Mark, D., Rashid, A., and Shariff, A. R. Natural-language spatial relations between linear and areal objects: The topology and metric of english-language terms. International Journal of Geographical Information Science, 12:215-246, 1998. 
Singh, G. and de By, R. A. Models for half-direction based part-whole relationships. In Proceedings of the third ACM SIGSPATIAL International Workshop on GeoStreaming (IWGS), page 9, California, USA, 2012. ACM.

Skiadopoulos, S. and Koubarakis, M. Composing cardinal direction relations. Artificial Intelligence, 152(2):143-171, 2004.

Stuckenschmidt, H., Schlieder, C., Visser, U., Vögele, T., and Neumann, H. Spatial reasoning for information brokering. In Proceedings of the Fourteenth International Florida Artificial Intelligence Research Society Conference, pages 568-573. AAAI Press, 2001. ISBN 1-57735-133-9.

Sullivan, M. Statistics: Informed Decisions Using Data. Pearson, U.S.A, 4th edition, 2008.

Sun, H. Computational models for computing fuzzy cardinal directional relations between regions. Knowledge-Based Systems, 21(7):599 - 603, 2008.

Theodoridis, Y., Papadias, D., and Stefanakis, E. Supporting direction relations in spatial database systems. In Proceedings of the 7th International Symposium on Spatial Data Handling (SDH)'96, 1996.

van Kreveld, M. J. and Reinbacher, I. Good NEWS: Partitioning a simple polygon by compass directions. International Journal of Computational Geometry \& Applications, 14:233-259, 2004.

van Rijsbergen, C. Information Retrieval. Butterworth, London, Boston, 1979.

Vestavik, Ø. Geographic information retrieval: An overview. In Proceedings of International ODRL workshop. 2004.

Voudouris, V., Fisher, P. F., and Wood, J. Capturing and representing conceptualization uncertainty interactively using object-fields. In Riedl, A., Kainz, W., and Elmes, G. A., editors, Progress in Spatial Data Handling, pages 755-770. Springer Berlin Heidelberg, 2006. ISBN 978-3-54035588-5.

Wallgrün, J. O., Frommberger, L., Wolter, D., Dylla, F., and Freksa, C. Qualitative spatial representation and reasoning in the sparq-toolbox. In Barkowsky, T., Knauff, M., Ligozat, G., and Montello, D. R., editors, Spatial Cognition \& Reasoning, Action, Interaction, volume 4387 of Lecture Notes in Computer Science, pages 39-58. Springer Berlin Heidelberg, 2007. ISBN 978-3-540-75665-1.

Werner, S., Krieg-Brückner, B., and Herrmann, T. Modelling navigational knowledge by route graphs. In Freksa, C., Habel, C., Brauer, W., and Wender, K. F., editors, Spatial Cognition II, volume 1849 of Lecture Notes in Computer Science, pages 295-316. Springer Berlin Heidelberg, 2000.

Wieczorek, J., Guo, Q., and Hijmans, R. J. The point-radius method for georeferencing locality descriptions and calculating associated uncertainty. International Journal of Geographical Information Science, 18(8):745-767, 2004. 
Wilcock, G. Introduction to Linguistic Annotation and Text Analytics. Morgan \& Claypool Publishers.

$\mathrm{Xu}$, J. Formalizing natural-language spatial relations between linear objects with topological and metric properties. Int. J. Geogr. Inf. Sci., 21(4):377-395, January 2007. ISSN 1365-8816.

$\mathrm{Xu}, \mathrm{J}$. and Mark, D. M. Natural language understanding of spatial relations between linear geographic objects. Spatial Cognition \& Computation, 7 (4):311-347, 2007.

Yamamoto, M., Takahashi, Y., Iwasaki, H., Oyama, S., Ohshima, H., and Tanaka, K. Extraction and geographical navigation of important historical events in the web. In Tanaka, K., Fröhlich, P., and Kim, K. S., editors, Web and Wireless Geographical Information Systems, volume 6574 of Lecture Notes in Computer Science, pages 21-35. Springer Berlin Heidelberg, 2011. ISBN 978-3-642-19172-5.

Zadeh, L. A. A simple view of the dempster-shafer theory of evidence and its implication for the rule of combination. Artificial Inteligence Magazine, 7:85-90, 1986.

Zhang, X., Mitra, P., Klippel, A., and MacEachren, A. Automatic extraction of destinations, origins and route parts from human generated route directions. In Fabrikant, S., Reichenbacher, T., Kreveld, M., and Schlieder, C., editors, Geographic Information Science, volume 6292 of Lecture Notes in Computer Science, pages 279-294. Springer Berlin Heidelberg, 2010. ISBN 978-3-642-15299-3.

Zhou, N. and Zhou, X. Automatic acquisition of linguistic patterns for conceptual modeling. Technical report, 2004.

Zimmermann, K. and Freksa, C. Qualitative spatial reasoning using orientation, distance, and path knowledge. Applied Intelligence, 6: 49-58, 1996. 



\section{Appendix}

This appendix contain Penn Treebank II tags from Bracketing Guidelines for Treebank II Style Penn Treebank Project Bies et al. (1995). Table A.1 five clause level tags and 21 phrase tags are shown in Table A.2. Word level tags and punctuation tags are shown in Table A.3 and Table A.4 respectively.

Table A.1 Clause level Penn Treebank Part-Of-Speech tags (taken from Bies et al. (1995))

\begin{tabular}{|c|l|l|}
\hline No. & Tag & Description \\
\hline 1 & S & $\begin{array}{l}\text { simple declarative clause, i.e., one that is not } \\
\text { introduced by a (possible empty) subordinating } \\
\text { conjunction or a wh-word and that does not exhi- } \\
\text { bit subject-verb inversion }\end{array}$ \\
\hline 2 & SBAR & $\begin{array}{l}\text { Clause introduced by a (possibly empty) subordi- } \\
\text { nating conjunction }\end{array}$ \\
\hline 3 & SBARQ & $\begin{array}{l}\text { Direct question introduced by a wh-word or a wh- } \\
\text { phrase. Indirect questions and relative clauses } \\
\text { should be bracketed as SBAR, not SBARQ }\end{array}$ \\
\hline 4 & SINV & $\begin{array}{l}\text { Inverted declarative sentence, i.e., one in which } \\
\text { the subject follows the tensed verb or modal }\end{array}$ \\
\hline 5 & SQ & $\begin{array}{l}\text { Inverted yes/no question, or main clause of a wh- } \\
\text { question, following the wh-phrase in SBARQ }\end{array}$ \\
\hline
\end{tabular}


Table A.2 Phrase level Penn Treebank Part-Of-Speech tags (taken from Bies et al. (1995))

\begin{tabular}{|c|c|c|}
\hline No. & Tag & Description \\
\hline 1 & ADJP & Adjective Phrase \\
\hline 2 & ADVP & Adverb Phrase \\
\hline 3 & CONJP & Conjunction Phrase \\
\hline 4 & FRAG & Fragment \\
\hline 5 & INTJ & $\begin{array}{l}\text { Interjection. Corresponds approximately to the } \\
\text { part-of-speech tag UH }\end{array}$ \\
\hline 6 & LST & List marker. Includes surrounding punctuation \\
\hline 7 & NAC & $\begin{array}{l}\text { Not a Constituent; used to show the scope of cer- } \\
\text { tain prenominal modifiers within an NP }\end{array}$ \\
\hline 8 & NP & Noun Phrase \\
\hline 9 & NX & $\begin{array}{l}\text { Used within certain complex NPs to mark the head } \\
\text { of the NP. Corresponds very roughly to N-bar level } \\
\text { but used quite differently }\end{array}$ \\
\hline 10 & $\mathrm{PP}$ & Prepositional Phrase \\
\hline 11 & PRN & Parenthetical \\
\hline 12 & PRT & $\begin{array}{l}\text { Particle. Category for words that should be tagged } \\
\text { RP }\end{array}$ \\
\hline 13 & QP & $\begin{array}{l}\text { Quantifier Phrase (i.e., complex measure/amount } \\
\text { phrase); used within NP }\end{array}$ \\
\hline 14 & RRC & Reduced Relative Clause \\
\hline 15 & UCP & Unlike Coordinated Phrase \\
\hline 16 & VP & Vereb Phrase \\
\hline 17 & WHADJP & $\begin{array}{l}\text { Wh-adjective Phrase. Adjectival phrase containing } \\
\text { a wh-adverb, as in how hot }\end{array}$ \\
\hline 18 & WHAVP & $\begin{array}{l}\text { Wh-adverb Phrase. Introduces a clause with an NP } \\
\text { gap. May be null (containing the } 0 \text { complemen- } \\
\text { tizer) or lexical, containing a wh-adverb such as } \\
\text { how or why }\end{array}$ \\
\hline 19 & WHNP & $\begin{array}{l}\text { Wh-noun Phrase. Introduces a clause with an NP } \\
\text { gap. May be null (containing the } 0 \text { complementi- } \\
\text { zer) or lexical, containing some wh-word, e.g., who, } \\
\text { which book, whose daughter, none of which, or } \\
\text { how many leopards }\end{array}$ \\
\hline 20 & WHPP & $\begin{array}{l}\text { Wh-prepositional Phrase. Prepositional phrase } \\
\text { containing a wh-noun phrase (such as of which } \\
\text { or by whose authority) that either introduces a PP } \\
\text { gap or is contained by a WHNP }\end{array}$ \\
\hline 21 & $\mathrm{X}$ & $\begin{array}{l}\text { Unknown, uncertain, or unbracketable. } \mathrm{X} \text { is often } \\
\text { used for bracketing typos and in bracketing the- } \\
\text { constructions }\end{array}$ \\
\hline
\end{tabular}


Table A.3 Word level Penn Treebank Part-Of-Speech tags (taken from Bies et al. (1995))

\begin{tabular}{|c|c|c|}
\hline No. & Tag & Description \\
\hline 1 & $\mathrm{CC}$ & Coordinating conjunction \\
\hline 2 & $\mathrm{CD}$ & Cardinal number \\
\hline 3 & DT & Determiner \\
\hline 4 & EX & Existential there \\
\hline 5 & FW & Foreign word \\
\hline 6 & IN & Preposition or subordinating conjunction \\
\hline 7 & JJ & Adjective \\
\hline 8 & JJR & Adjective, comparative \\
\hline 9 & JJS & Adjective, superlative \\
\hline 10 & $\mathrm{LS}$ & List item marker \\
\hline 11 & MD & Modal \\
\hline 12 & NN & Noun, singular or mass \\
\hline 13 & NNS & Noun, plural \\
\hline 14 & NNP & Proper noun, singular \\
\hline 15 & NNPS & Proper noun, plural \\
\hline 16 & PDT & Predeterminer \\
\hline 17 & POS & Possessive ending \\
\hline 18 & PRP & Personal pronoun \\
\hline 19 & PRPS & Possessive pronoun \\
\hline 20 & $\mathrm{RB}$ & Adverb \\
\hline 21 & RBR & Adverb, comparative \\
\hline 22 & RBS & Adverb, superlative \\
\hline 23 & RP & Particle \\
\hline 24 & SYM & Symbol \\
\hline 25 & TO & to \\
\hline 26 & $\mathrm{UH}$ & Interjection \\
\hline 27 & VB & Verb, base form \\
\hline 28 & VBD & Verb, past tense \\
\hline 30 & VBN & Verb, past participle \\
\hline 31 & VBP & Verb, non-3rd person singular present \\
\hline 32 & VBZ & Verb, 3rd person singular present \\
\hline 33 & WDT & Wh-determiner \\
\hline 34 & WP & Wh-pronoun \\
\hline 35 & WP\$ & Possessive wh-pronoun \\
\hline 36 & WRB & Wh-adverb \\
\hline
\end{tabular}


Table A.4 Punctuation tags under Penn Treebank tagset (taken from Bies et al. (1995))

\begin{tabular}{|c|l|l|}
\hline Punctuation & Tag & Description \\
\hline$\#$ & $\#$ & Hash symbol \\
\hline (, \{ and [ & -LRB- & Open round, curly and square bracket \\
\hline ), \} and ] & -RRB- & Closed round, curly and square bracket \\
\hline$\$$ & $\$$ & Currency symbol \\
\hline " & " & Open double quote \\
\hline$"$ & $"$ & Closed double quote \\
\hline$\cdot$ & ' & Open single quote \\
\hline, & & Closed single quote \\
\hline, &, & Comma \\
\hline$\cdot$ & $\cdot$ & Dot \\
\hline$:$ & $:$ & Colon \\
\hline$;$ & $;$ & Semicolon \\
\hline
\end{tabular}




\section{Appendix}

Table B.1: Tregex node-node relations and their symbols

\begin{tabular}{|c|c|}
\hline Symbol & Meaning \\
\hline $\mathrm{A}<<\mathrm{B}$ & A dominates B \\
\hline $\mathrm{A}>>\mathrm{B}$ & $\mathrm{A}$ is dominated by $\mathrm{B}$ \\
\hline $\mathrm{A}<\mathrm{B}$ & A immediately dominates B \\
\hline $\mathrm{A}>\mathrm{B}$ & A is immediately dominated by $\mathrm{B}$ \\
\hline$A \$ B$ & $\mathrm{~A}$ is a sister of $\mathrm{B}$ (and not equal to $\mathrm{B}$ ) \\
\hline A.. B & A precedes B \\
\hline A. B & A immediately precedes B \\
\hline$A, B$ & A follows B \\
\hline$A, B$ & A immediately follows B \\
\hline $\mathrm{A}<<, \mathrm{B}$ & $\mathrm{B}$ is a leftmost descendant of $\mathrm{A}$ \\
\hline $\mathrm{A}<<-\mathrm{B}$ & $\mathrm{B}$ is a rightmost descendant of $\mathrm{A}$ \\
\hline $\mathrm{A}>>, \mathrm{B}$ & $\mathrm{A}$ is a leftmost descendant of $\mathrm{B}$ \\
\hline$A>>-B$ & $\mathrm{~A}$ is a rightmost descendant of $\mathrm{B}$ \\
\hline $\mathrm{A}<, \mathrm{B}$ & $\mathrm{B}$ is the first child of $\mathrm{A}$ \\
\hline$A>, B$ & $A$ is the first child of $B$ \\
\hline $\mathrm{A}<-\mathrm{B}$ & $\mathrm{B}$ is the last child of $\mathrm{A}$ \\
\hline$A>-B$ & $A$ is the last child of $B$ \\
\hline $\mathrm{A}<{ }^{\prime} \mathrm{B}$ & $B$ is the last child of $A$ \\
\hline$A>{ }^{\prime} B$ & $\mathrm{~A}$ is the last child of $\mathrm{B}$ \\
\hline $\mathrm{A}<\mathrm{i} B$ & $\mathrm{~B}$ is the ith child of $\mathrm{A}(\mathrm{i}>0)$ \\
\hline $\mathrm{A}>\mathrm{i} \mathrm{B}$ & $\mathrm{A}$ is the ith child of $\mathrm{B}(\mathrm{i}>0)$ \\
\hline$A<-i B$ & $\mathrm{~B}$ is the ith-to-last child of $\mathrm{A}(\mathrm{i}>0)$ \\
\hline$A>-i B$ & $\mathrm{~A}$ is the ith-to-last child of $\mathrm{B}(\mathrm{i}>0)$ \\
\hline $\mathrm{A}<\mathrm{B}$ & $\mathrm{B}$ is the only child of A \\
\hline $\mathrm{A}>\mathrm{B}$ & $\mathrm{A}$ is the only child of $\mathrm{B}$ \\
\hline $\mathrm{A}<<: \mathrm{B}$ & $\begin{array}{l}\text { A dominates B via an unbroken chain (length }>0 \text { ) } \\
\text { of unary local trees }\end{array}$ \\
\hline $\mathrm{A}>>: \mathrm{B}$ & $\begin{array}{l}\text { A is dominated by B via an unbroken chain (length } \\
>0 \text { ) of unary local trees }\end{array}$ \\
\hline $\mathrm{A} \$++\mathrm{B}$ & $\begin{array}{l}\text { A is a left sister of B (same as } \$ . . \text { for context-free } \\
\text { trees) }\end{array}$ \\
\hline
\end{tabular}




\begin{tabular}{|l|l|}
\hline A \$- B & $\begin{array}{l}\text { A is a right sister of B (same as \$, for context-free } \\
\text { trees) }\end{array}$ \\
\hline A \$+ B & $\begin{array}{l}\text { A is the immediate left sister of B (same as \$. for } \\
\text { context-free trees) }\end{array}$ \\
\hline A \$- B & $\begin{array}{l}\text { A is the immediate right sister of B (same as \$, for } \\
\text { context-free trees) }\end{array}$ \\
\hline A \$.. B & A is a sister of B and precedes B \\
\hline A \$, B & A is a sister of B and follows B \\
\hline A \$. B & A is a sister of B and immediately precedes B \\
\hline A \$, B & A is a sister of B and immediately follows B \\
\hline A $<+(C)$ B & $\begin{array}{l}\text { A dominates B via an unbroken chain of (zero or } \\
\text { more) nodes matching description C }\end{array}$ \\
\hline A $>+(C)$ B & $\begin{array}{l}\text { A is dominated by B via an unbroken chain of (zero } \\
\text { or more) nodes matching description C }\end{array}$ \\
\hline A .+(C) B & $\begin{array}{l}\text { A precedes B via an unbroken chain of (zero or } \\
\text { more) nodes matching description C }\end{array}$ \\
\hline A ,+(C) B & $\begin{array}{l}\text { A follows B via an unbroken chain of (zero or } \\
\text { more) nodes matching description C }\end{array}$ \\
\hline A <<\# B & B is a head of phrase A \\
\hline A $>>\#$ B & A is a head of phrase B \\
\hline A <\# B & B is the immediate head of phrase A \\
\hline A $>\#$ B & A is the immediate head of phrase B \\
\hline A == B & A and B are the same node \\
\hline A : B & $\begin{array}{l}\text { [this is a pattern-segmenting operator that places } \\
\text { no constraints on the relationship between A and } \\
\text { B] }\end{array}$ \\
\hline & \\
\hline
\end{tabular}




\section{Appendix}

Table C.1 and thereafter are the main contribution of our work in Chapter 3 in extracting information from various spatial hints from our gazetteer corpus.

Table C.1 Modularised parts of SHLP

\begin{tabular}{|c|c|}
\hline Modular part & Expression \\
\hline$\overline{\mathrm{VAL}}$ & $/^{\prime \wedge}[0-9,-]+\$ /$ \\
\hline DUNI & km | mi \\
\hline EUNI & \begin{tabular}{l|l}
$\mathrm{m}$ & $\mathrm{ft}$
\end{tabular} \\
\hline APPROX & /[Cc]irca/ \\
\hline CDIR & $\begin{array}{l}\text { NW | NE | SE | SW | N | E | W | S | NNE | ENE | ESE | SSE | SSW } \\
\text { | WSW | WNW | NNW }\end{array}$ \\
\hline DIRRLE & $\begin{array}{l}\text { upper | middle | lower | uppermost | lowermost | } \\
\text { eastern | western | southern | northern }\end{array}$ \\
\hline RELDIR & above | below \\
\hline CHAR & 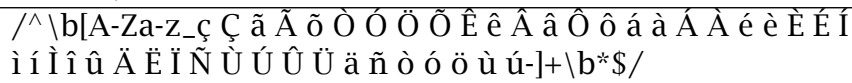 \\
\hline GEOCODE & $/^{\wedge}[0-9]+N ? \backslash \backslash \backslash /[0-9]+\$ /$ \\
\hline ORG & $/^{\wedge} \mid \mathrm{b}[\mathrm{A}-\mathrm{Z}]+\backslash \mathrm{b} * \$ /$ \\
\hline PARTOF & $\begin{array}{l}\text { center | central | eastern | western | northern } \\
\text { | southern | northeastern | northwestern | } \\
\text { southeastern | southwestern }\end{array}$ \\
\hline PRE & extreme | central \\
\hline CAT & state $\mid$ city | town \\
\hline EXT & end $\mid$ side $\mid$ border $\mid$ slope \\
\hline
\end{tabular}

Below we provide the Spatial Hint Linguistic Patterns (SHLP) used for extracting information from spatial hints present in our gazetteer entries.

\section{P1 - Elevation}

Example: $200 \mathrm{~m}$

$N P<(((C D<V A L) ! \$ / N N . ? /<$ APPROX $) \$+(/ N N$. ?/ [EUNI] $))$

P2 - Approximate elevation

Example: Ca. $200 \mathrm{~m}$

$N P<1(/ N N . ? /<$ APPROX $)$ 
$<2(\mathrm{CD}<\mathrm{VAL})$

$<3$ (/NN.?/ [EUNI])

P3 - Place name and geocode

Example: Manoá [0940/6527 (USBGN)]

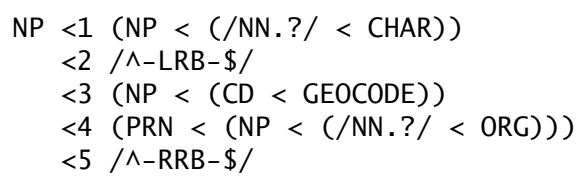

P4 - Place name, alternate place name and geocode

Example: Erexim [Erechim, 0940/6527 (USBGN)]

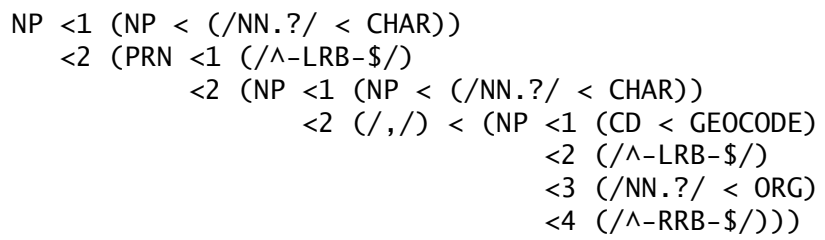

$<3$ /^-RRB- $\$$ /)

P5 - Distance direction from a reference point

Example 1: $47 \mathrm{~km}$ NE Ponte Nova [2024/4254 (USBGN)]

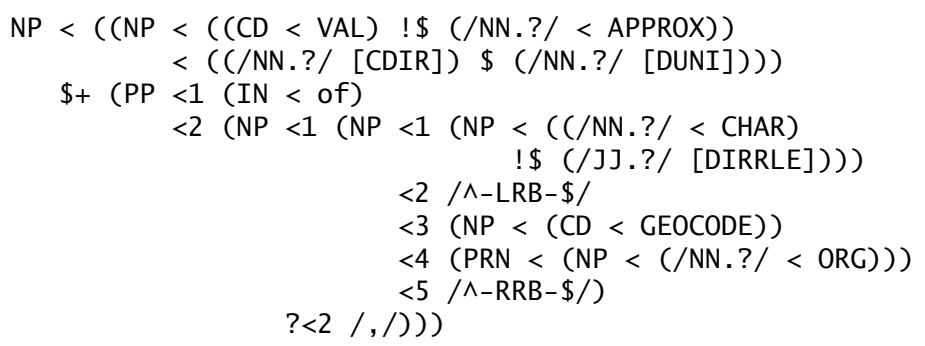

Example 2: 60 km WNW of city of São Paulo [2332/4637 (USBGN)]

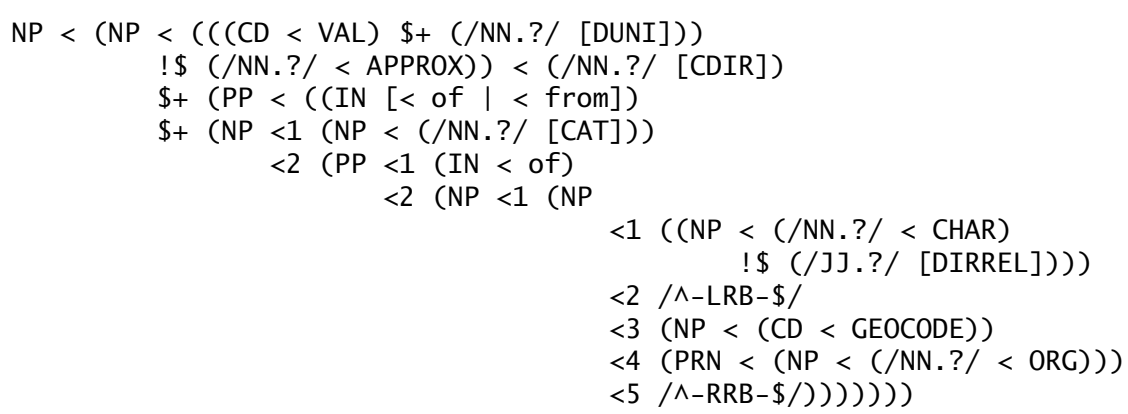

P6 - Approximate distance direction from a reference point Example 1: ca. 150 km SSE of Belém [0127/4829 (USBGN)])

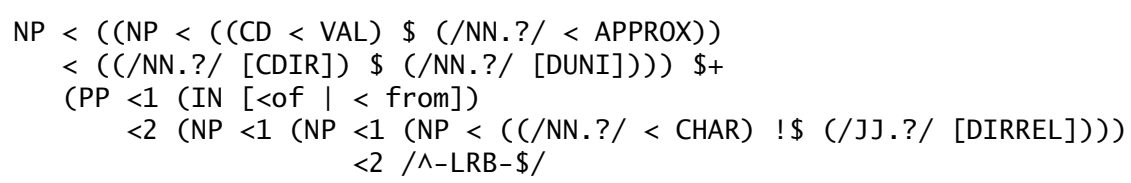




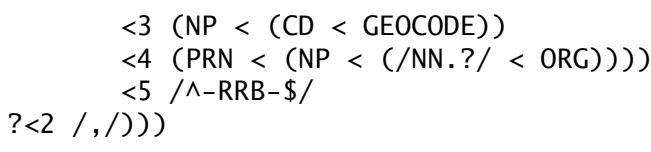

Example 2: ca. 50 km W of city of São Paulo [2332/4637 (USBGN)]

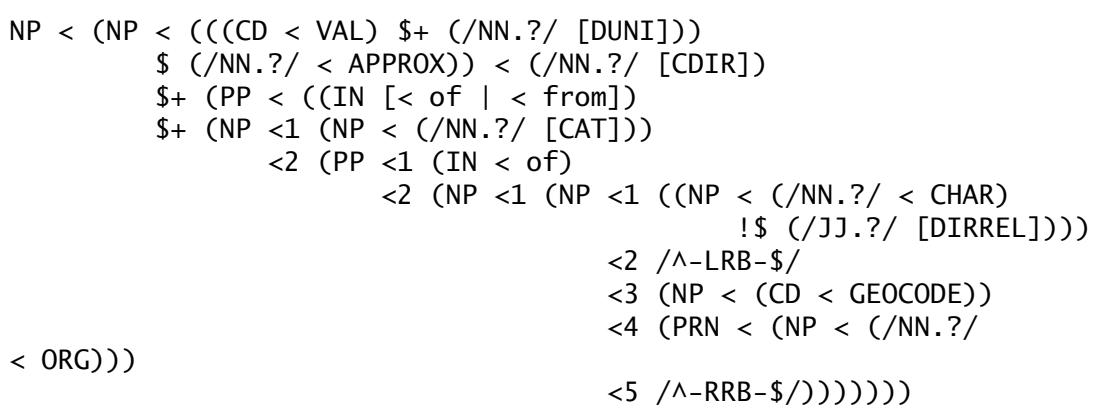

P7 - Distance direction (above or below) from a reference point

Example 1: $25 \mathrm{~km}$ above mouth of Rio Madeira [0322/5845 (USBGN)]

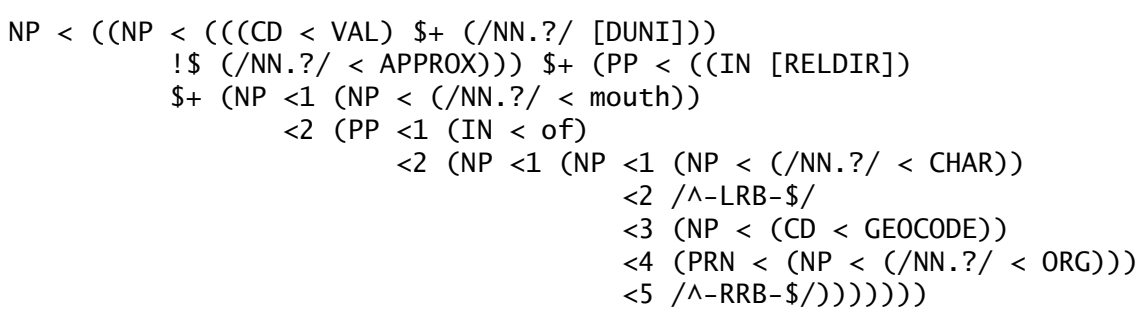

Example 2: ca. 25 km below mouth of Rio Cuiuni [0045/6307 (USBGN)]

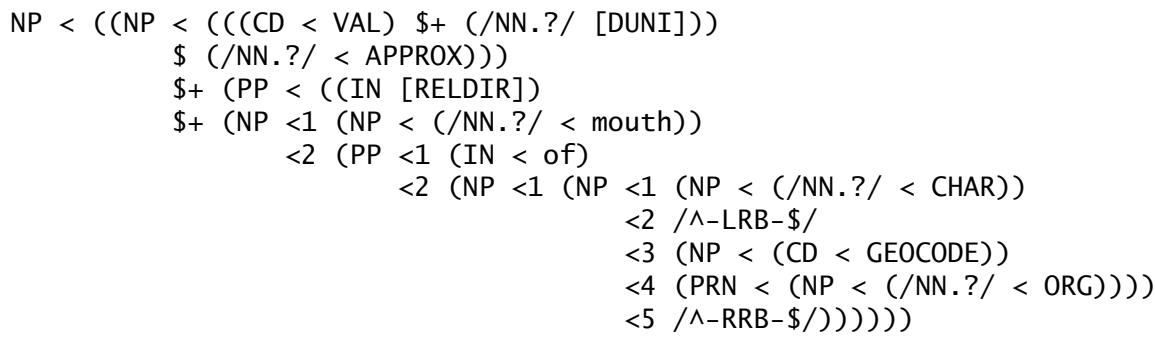

P8 - Distance direction (above or below) from a reference point Example 1: $200 \mathrm{~km}$ above Manaus [0308/5845 (USBGN)]

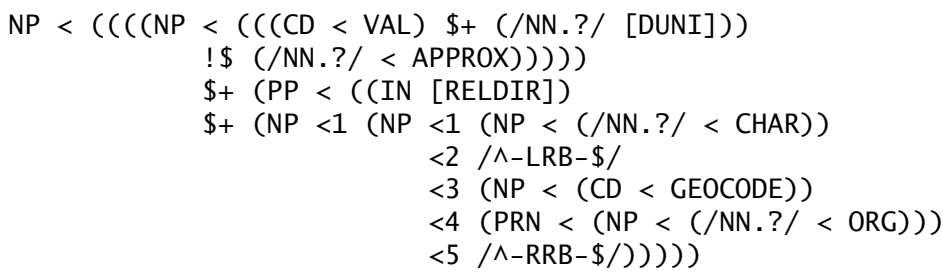

Example 2: ca. 25 km below Tôrres [2921/4944 (USBGN)] 


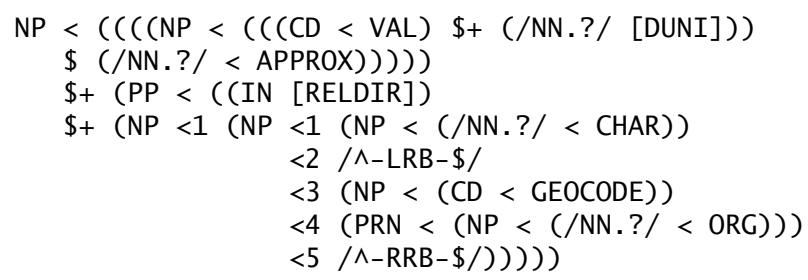

\section{P9 - Direction offset}

Example 1: N of Uberaba [1945/4755 (USBGN)]

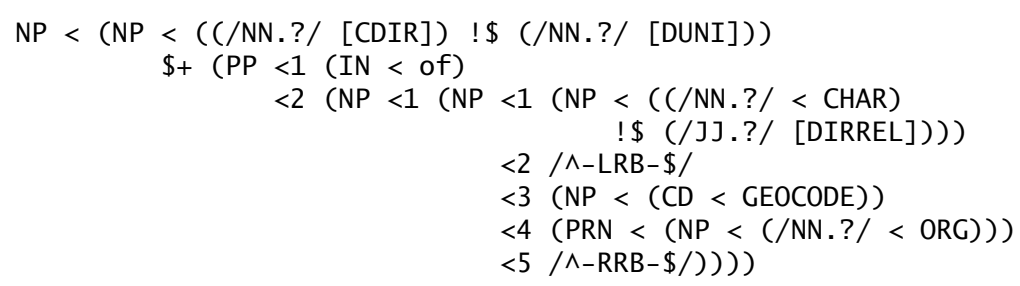

Example 2: N of town of Garopaba [2804/4840 (USBGN)]

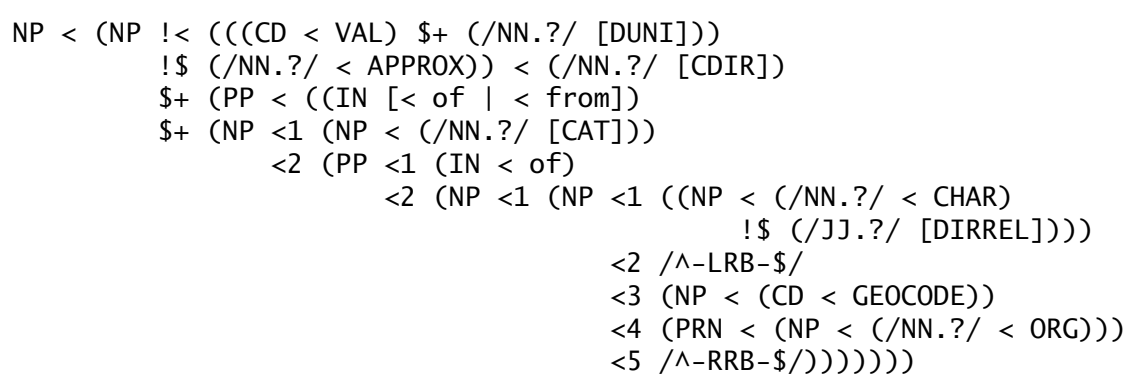

Example 3: N of lower Rio São Mateus [1835/3944 (USBGN)]

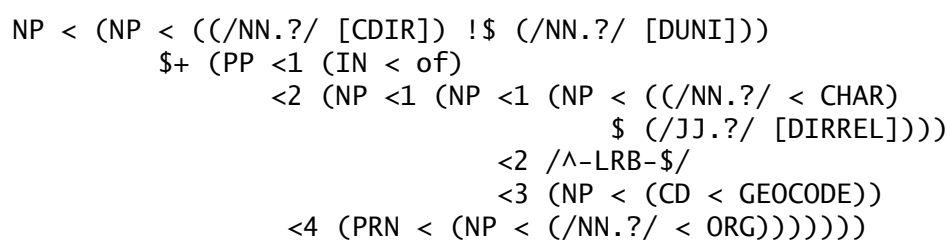

Example 4: above Linhares [1925/4004 (USBGN)]

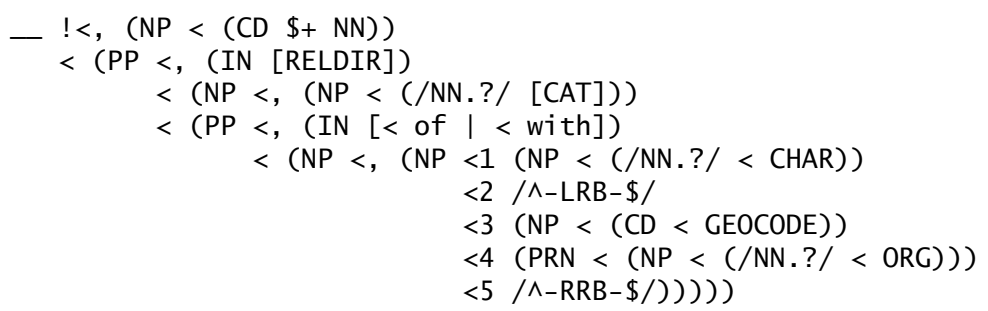

Example 5: below town of Arapari [0859/5711 (USBGN)] 


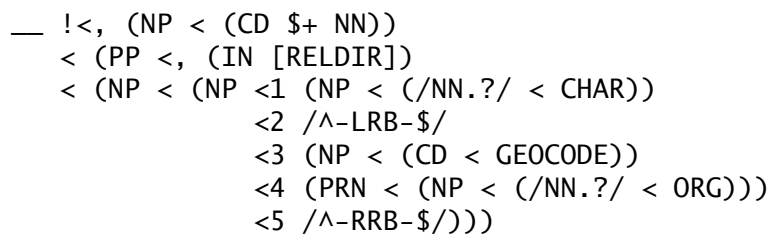

Example 6: above mouth of Ribeirão da Vereda [1518/4053 (USBGN)]

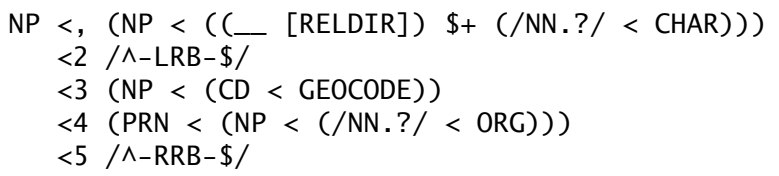

P10 - Between-two-features

Example 1: between Uaupes [0008/6705 (USBGN)] and Tapurucuara [0024/6502 (USBGN)]

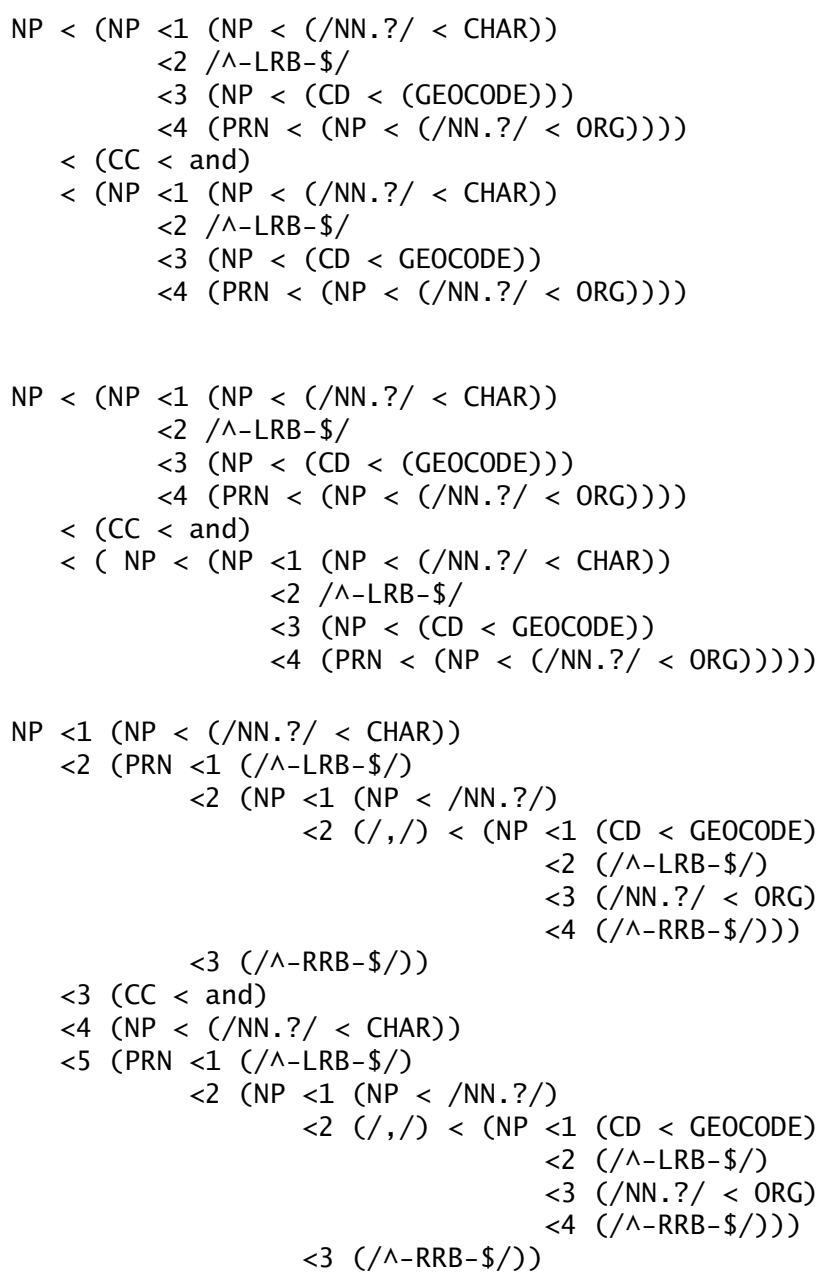


Example 2: between Lorena [2244/4508 (USBGN)] and Cachoeira [Cachoeira Paulista, 2240/4501 (USBGN)]

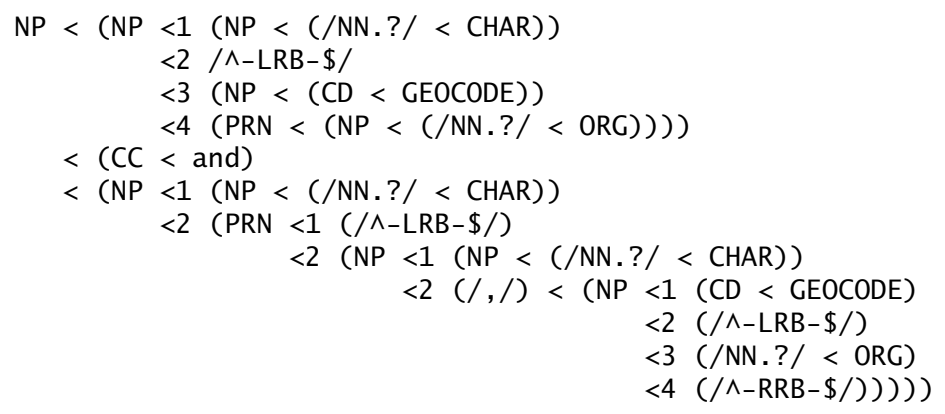

\section{P11 - Direction part of region}

Example 1: northeastern <region>

$\begin{aligned} N P & <((J][P A R T O F]) \$(J][P R E])) \\ & <(/ N N . ? /<\text { CHAR })\end{aligned}$

Example 2: central northern <region> and extreme northwestern $<$ region $>$

$\begin{aligned} \mathrm{NP} & <((\mathrm{J}][\mathrm{PARTOF}]) \$+(J][\mathrm{PRE}])) \\ & <(/ \mathrm{NN} . ? /<\mathrm{CHAR})\end{aligned}$

Example 3: northern central <region>

$\mathrm{NP}<, \quad((\mathrm{J}]$ [PARTOF] $) ! \$+(\mathrm{J}]$ [PARTOF] $))$
$! \$(/ \mathrm{NN} . ? /$ [EXT] $)$

! \$ (/NN.?/ [EXT])

$<$ (/NN.?/ < CHAR) 


\section{Biography}

Gaurav Singh received his bachelor's degree in Civil engineering with distinction at North Maharashtra University, India in 2002. After that he worked for SPAN consultant (now a part of SNCLavalin group) as a Highway/GIS engineer. In 2003 he joined M.Sc in GeoInformatics course at Indian Institute of Remote Sensing (IIRS) which was a joint program with International Institute for

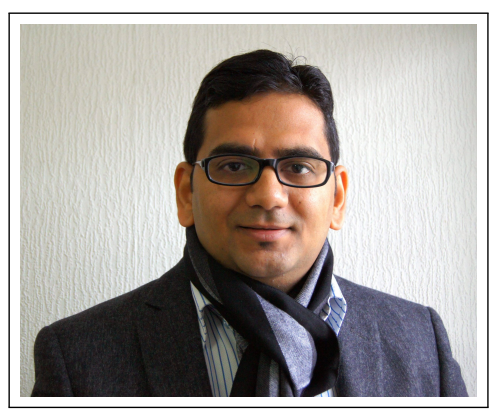
Geo-Information Science and Earth Observation (ITC). He graduated and obtained ITC M.Sc degree in 2004 with thesis work on "Framework for Location Based Emergency Services in India". Then he worked first in the capacity of GIS engineer at Rolta India Ltd. working with the Geospatial consultancy department and later as a Technical consultant for Civil and Geo products at Bentley Systems. In June 2009, after five years of working on various projects for the government and providing training to various organizations, he received funding from European Commission Erasmus Mundus program to pursue $\mathrm{PhD}$ at Geo-Information Processing department at ITC under the leadership of Prof. dr. Menno-Jan Kraak and supervision of Dr. ir. Rolf A. de By on translating Location description text in to a map. This document is the result of his work and is also based on following scientific publications.

- G. Singh and R. A. de By. (2014) Spatial extent models for natural language phrases involving directional containment. In: Transactions in GIS (Accepted for publication).

- G. Singh and R. A. de By. (2012) Models for Half-Direction based Part-Whole Relationships. In: ACM SIGSPATIAL IWGSŠ12, November 6, 2012. Redondo Beach, California, USA. ISBN: 978-1-4503-16958/12/11. 9 p.

- G. Singh and R. A. de By. (2012) Interpretation models for nonstandard compass directions. In: Extended Abstracts: Proceedings 
7th International Conference on Geographic Information Science, Columbus, OH, U.S.A.

- G. Singh, R. A. de By, and I. Ivánová. (2012) Concepts,compass and computation: Models for directional part-whole relationships. In B. Murgante, O. Gervasi, S. Misra, N. Nedjah, A. M. A. Rocha, D. Taniar, and B. O. Apduhan, editors, Computational Science and Its Applications (ICCSA) 2012, volume 7334 of Lecture Notes in Computer Science, pages 286-301. Springer, 2012.

- G. Singh and R. A. de By. (2011) The Extraction of Spatial Information from Natural Language Descriptions: The case of a Historical Gazetteer. Poster presented in: Reasoning Web Summer School, August 23-27, 2011, Galway, Ireland. 


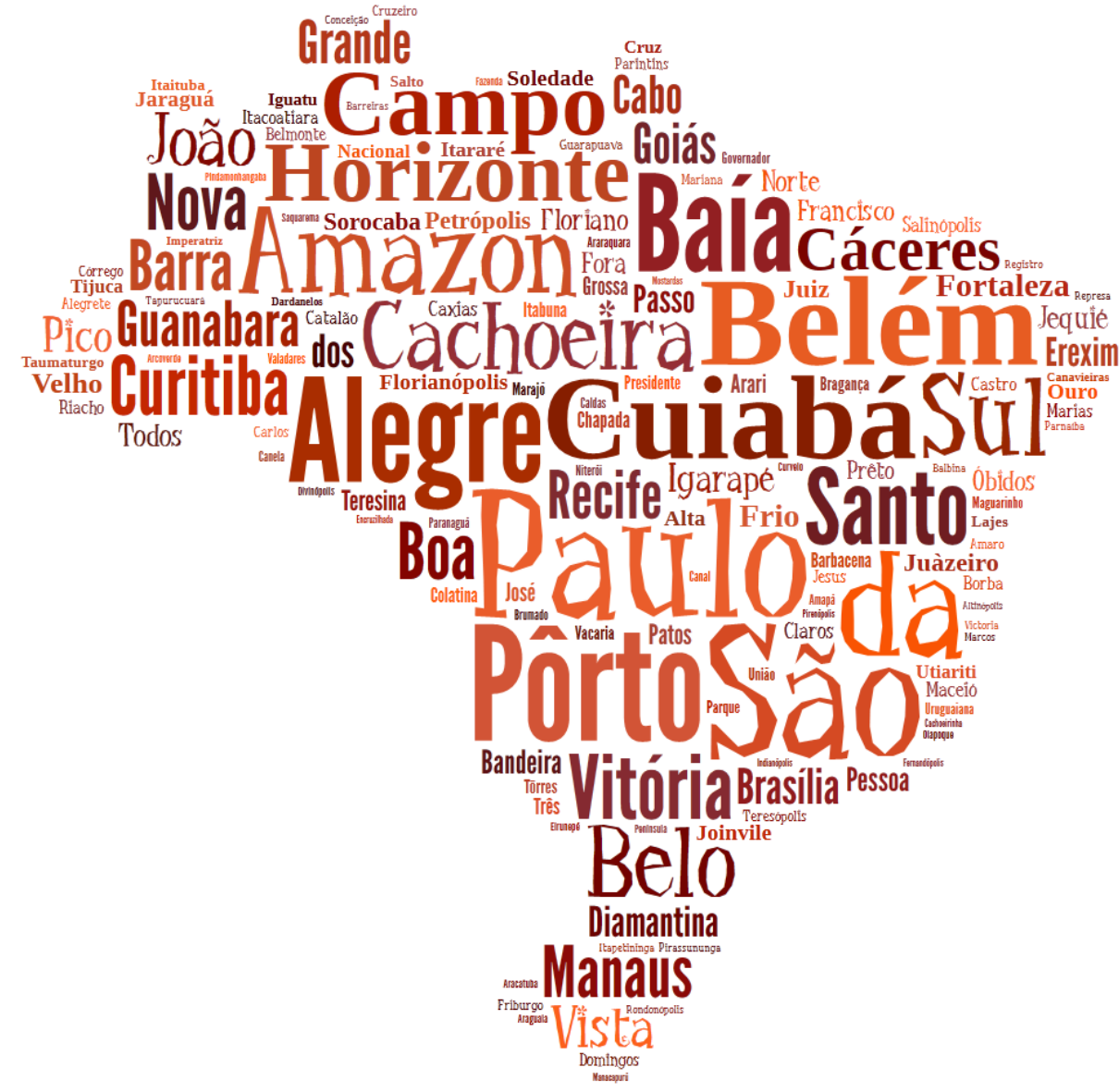

